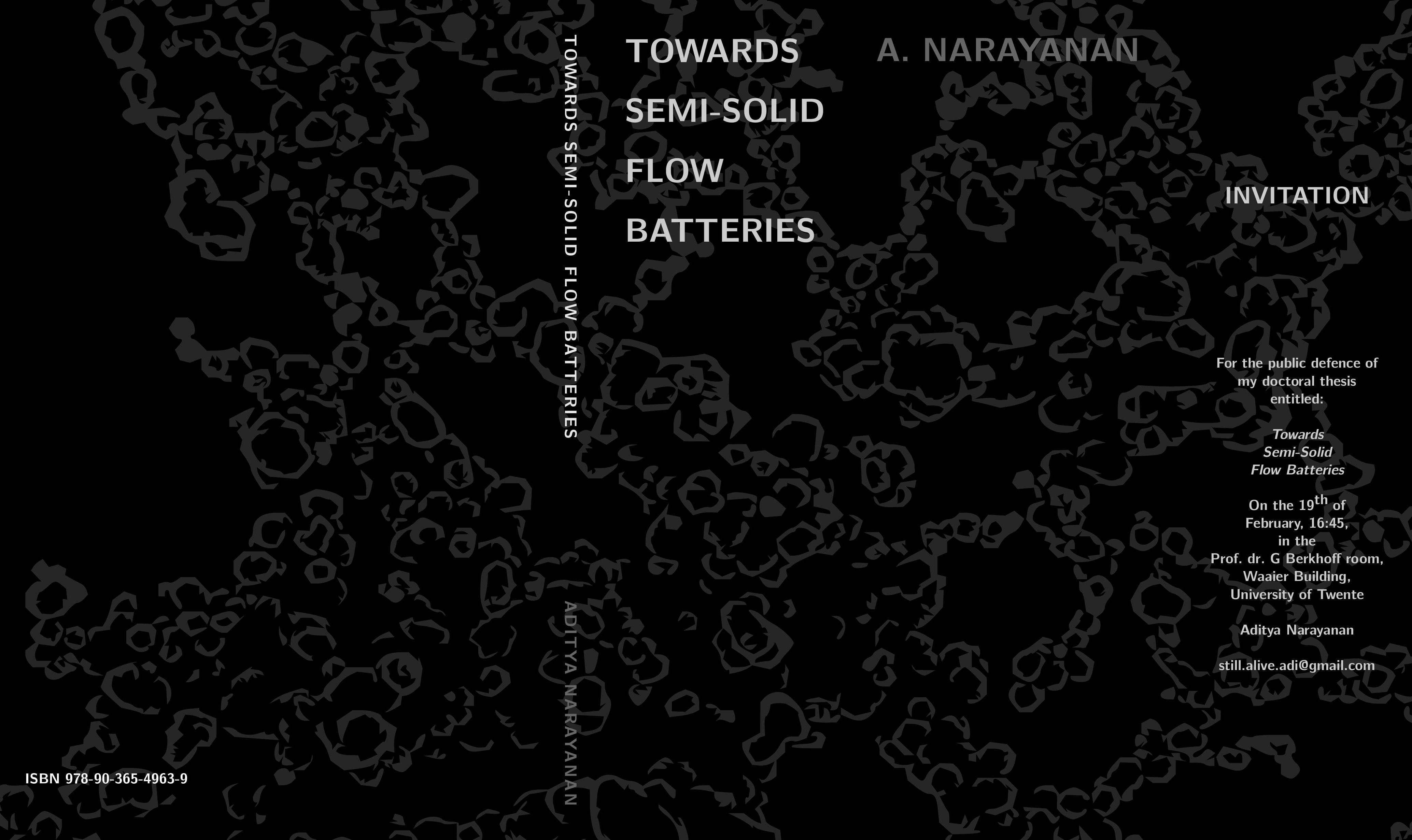




\section{Towards Semi-Solid Flow Batteries}

Aditya Narayanan 


\section{Graduation Committee}

$\begin{array}{lll}\text { Prof. Dr. J.L. Herek } & \text { University of Twente } & \text { Chairman } \\ \text { Prof. Dr. F. Mugele } & \text { University of Twente } & \text { Promotor } \\ \text { Dr. M.H.G. Duits } & \text { University of Twente } & \text { Assistant Promotor }\end{array}$

\section{Members}

Prof. Dr. Ir. P.D. Anderson

Prof. Dr. M.M.A.E. Claessens

Dr. Ir. W. Olthuis

Prof. Dr. A.P. Philipse

\author{
Eindhoven University of Technology \\ University of Twente \\ University of Twente \\ Utrecht University
}

The research reported in this thesis was carried out at the Physics of Complex Fluids (PCF) group within the MESA+ institute for Nanotechnology and the Department of Science and Technology of the University of Twente. The research leading to these results has received funding from the European Union Seventh Framework Programme (FP7/2007-2013) under grant agreement nº 608621.

Title:

Author:

ISBN:

DOI:
Towards Semi-Solid Flow Batteries

Aditya Narayanan

978-90-365-4963-9

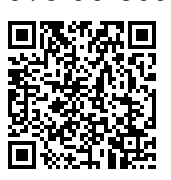

$10.3990 / 1.9789036549639$

Copyright $\odot 2020$ A. Narayanan 


\title{
TOWARDS SEMI-SOLID FLOW
}

\section{BATTERIES}

\section{DISSERTATION}

\author{
To obtain \\ the degree of doctor at the University of Twente, \\ on the authority of the rector magnificus, \\ Prof. dr. T.T.M. Palstra, \\ on account of the decision of the Doctorate Board, \\ to be publicly defended \\ on Wednesday, 19 February 2020, 16:45 \\ by
}

\section{Aditya Narayanan}

Born 11 March 1990 in Bangalore, India 
This dissertation has been approved by:

Prof. dr. F. Mugele (Promotor)

Dr. M. H. G. Duits (Assistant promotor) 


\section{Contents}

1 Introduction 1

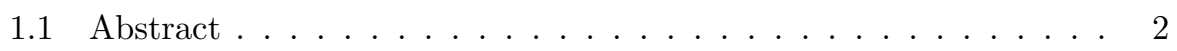

1.2 Introduction . . . . . . . . . . . . . . . . . . 3

1.2.1 Battery Architectures . . . . . . . . . . . . 3

1.3 Motivation: The Move to Fluid Electrodes . . . . . . . . . . . . 7

1.4 Thesis Outline ..................... 8

2 Colloidal Interactions $\quad 11$

2.1 Abstract ............................ 12

2.2 Introduction . . . . . . . . . . . . . . . . . . 13

2.3 Theory.......................... 16

2.4 Experiments . . . . . . . . . . . . . . . . . 17

2.4.1 Materials and sample preparation . . . . . . . . . 17

2.4.2 Measurement of hydrodynamic radius and zeta potential . . 18

2.5 Results and Discussion . . . . . . . . . . . . . 20

2.5.1 KB in pure EC PC solvent . . . . . . . . . . . 20

2.5.2 KB in electrolyte solutions ............. . . 23

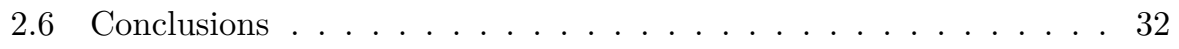




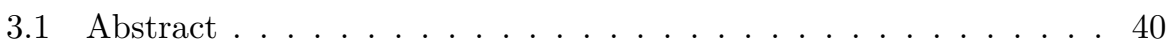

3.2 Introduction . . . . . . . . . . . . . . . . . . . . 41

3.3 Materials and Methods . . . . . . . . . . . . . . . . 44

3.3.1 Suspension Preparation ............... 44

3.3.2 Rheoimpedance Measurements . . . . . . . . . . . . . 45

3.3.3 Impedance Analysis . . . . . . . . . . . . . . . . . . 48

3.4 Results and Discussion . . . . . . . . . . . . . . . . 50

3.4.1 Intrinsic Flow curves . . . . . . . . . . . . . . 50

3.4.2 Transient behaviors ................ 52

3.4.3 Mechanistic Picture ................ 53

3.4 .4 Modeling .................... 58

3.4.5 Cessation of shear ................. 59

3.5 Conclusion .......................... 62

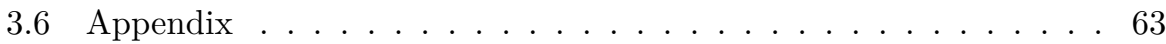

3.7 Supplementary Information . . . . . . . . . . . . . . 68

4 Cycling-Rheoimpedance Device $\quad 77$

4.1 Abstract . . . . . . . . . . . . . . . . 78

4.2 Introduction . . . . . . . . . . . . . . . . . . . 78

4.3 Global Setup . . . . . . . . . . . . . . . . 80

4.3 .1 Detailed Description . . . . . . . . . . . 82

4.3.2 Assembly and Sample loading . . . . . . . . . . . . 84

4.3.3 Rheological Verification .............. 85

4.4 Application to flow systems . . . . . . . . . . . . 85

4.4.1 SSFB fluid electrodes in half-cell configuration . . . . . . 86 
4.4.2 FSC fluid electrode in full-cell configuration . . . . . . . . 88

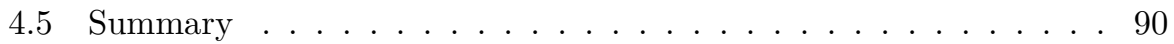

4.6 Supplementary Information _. . . . . . . . . . . . . 90

4.6.1 Materials and sample preparation . . . . . . . . . . 90

4.6.2 Electrochemical measurements and control . . . . . . . . 91

4.6.3 Rheoimpedance protocol . . . . . . . . . . . . 92

4.6.4 Stress Strain Curves (Yield Stress) . . . . . . . . . . 93

4.6.5 Impedance Spectra . . . . . . . . . . . . . . . . . . . . 93

5 Electrochemical history dependence $\quad 97$

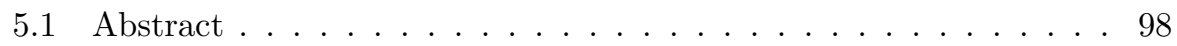

5.2 Introduction . . . . . . . . . . . . . . . . 99

5.3 Materials and Methods . . . . . . . . . . . . . . . 102

5.3.1 Fluid Electrode Preparation . . . . . . . . . . . . . 102

5.3.2 Cycling-rheo-impedance setup . . . . . . . . . . . 102

5.3.3 Experimental Protocol . . . . . . . . . . . . . . 105

5.4 Results and Discussion . . . . . . . . . . . . . . 106

5.5 Conclusions ............................ 116

5.6 Supplementary Information . . . . . . . . . . . . . 117

6 Surface Layers

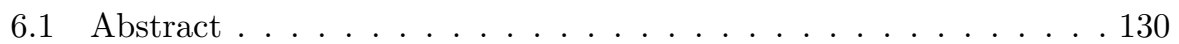

6.2 Introduction . . . . . . . . . . . . . . . . . . . . 131

6.3 Materials and Methods . . . . . . . . . . . . . . . . . 134

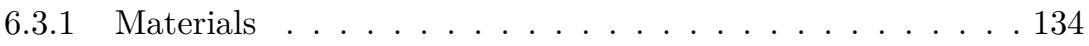

6.3.2 Sensor Preparation . . . . . . . . . . . . . 134 
6.3.3 EQCM Cell . . . . . . . . . . . . . 135

6.4 QCM-D Analysis . . . . . . . . . . . . . 136

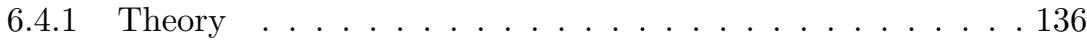

6.4.2 Model Fitting . . . . . . . . . . . . . . . 137

6.5 Results and Discussion . . . . . . . . . . . . . . . . 140

6.5.1 Measured QCM-D signals and model fits . . . . . . . . . . 140

6.5.2 Global changes in mass and viscoelastic compliance . . . . 142

6.5.3 Changes per Voltage cycle . . . . . . . . . . . . . 144

6.5.4 Correlation between changes in mass and compliance . . . . 149

6.5.5 Cycling of Carbon directly to $0 \mathrm{~V} \ldots \ldots . . . . . . .151$

6.5.6 Cycling of Carbon in EC:DMC electrolyte . . . . . . . 153

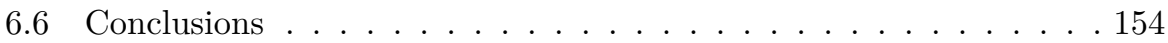

6.7 Supplementary Information . . . . . . . . . . . . . . . 155

6.7.1 Accuracy and robustness of the viscoelastic modeling. . . . 155

6.7 .2 Other Graphs . . . . . . . . . . . . . . 164

7 Conclusions and Outlook

7.1 Conclusions . . . . . . . . . . . . . . . . . . . . 174

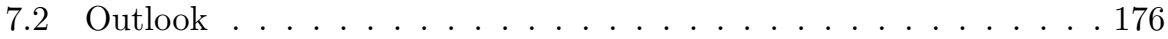

Summary

179

Samenvatting

182

Acknowledgements

185

TL;DR

188 
Publications

About the Author

190 

Chapter 1

\section{Introduction}

Aditya NARAyAnAN 


\subsection{Abstract}

The shift to renewable energy production has created new demands on the electric grid for balancing production with demand. Batteries are seen as one of many solutions for matching quick changes in electric power demand. The Semi-Solid Flow Battery (SSFB), a lithium ion based flow battery, with fluidic electrodes in the form of particle slurries is seen as a promising candidate to fill this niche ${ }^{1,2,5}$. It offers unique advantages in terms of energy density, scalability and long term operation. While much research has been done on optimizing traditional Lithium Ion Batteries (LIBs) and chemistries, translating to a flow architecture presents unique challenges and poses unanswered questions. Slurry electrodes are characterized by a (non-equilibrium) microstructure (the arrangement of the dispersed particles) which can vary, depending on the acting forces and their history. Conversely, this structure at the microscale can have a significant influence on macroscopic properties.

In this thesis, we study the impact of colloidal, mechanical and electrochemical effects on SSFBs and their fluid electrodes. In this chapter, we provide some background, outline the motivations for this study and discuss the challenges of realizing SSFBs. We also give a brief introduction to the aspects of batteries that are most relevant to this thesis. Additionally we provide an outline of the other thesis chapters. 


\subsection{Introduction}

\subsubsection{Battery Architectures}

\section{The Lithium ion Battery}

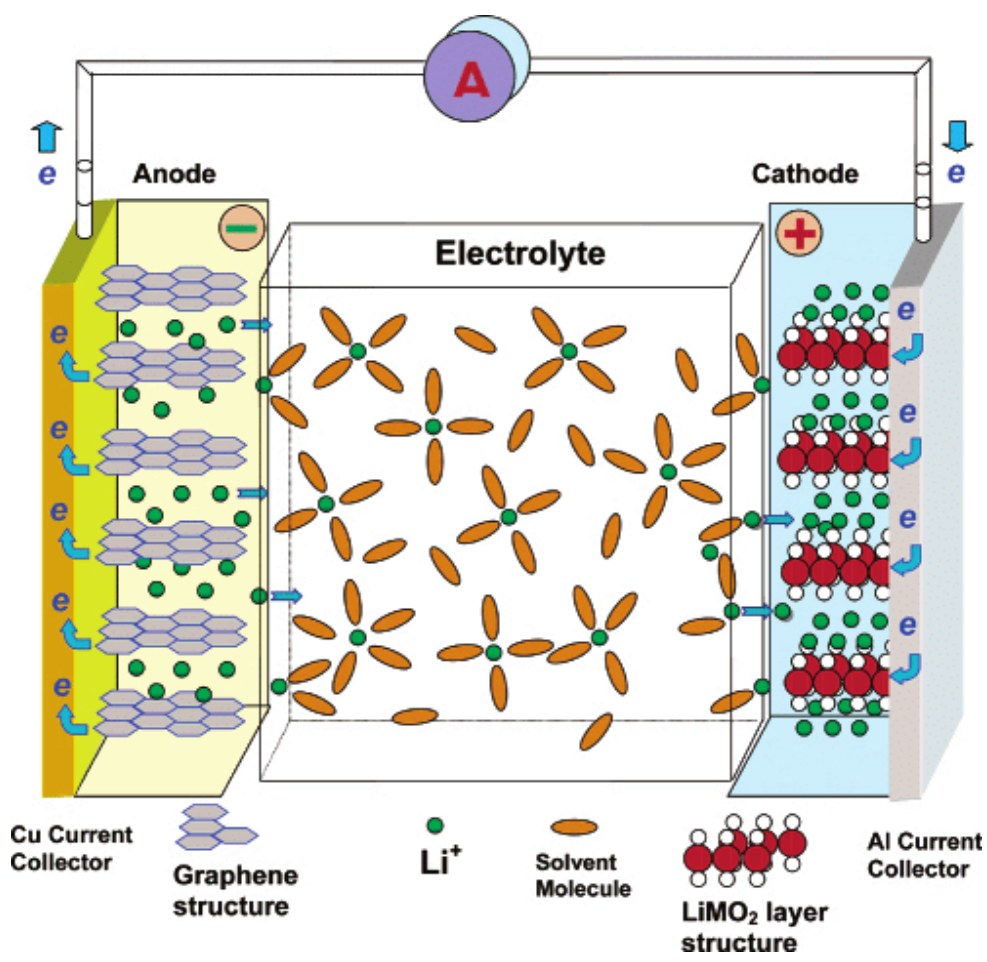

Figure 1.1: Cartoon of a rocking chair lithium ion battery. Reprinted with permission from Ref ${ }^{6}$. Copyright (02004 American Chemical Society

LIBs first demonstrated in the 1970s and commercialized in the 1990s are ubiquitous today; from smartphones to electric cars. While a plethora of different chemistries exist, most commercial LIBs are 'rocking chair' batteries (Fig.1.1), so called because the charge carriers shuttle back and forth. These batteries 
consist of two electrodes, an anode and cathode, separated by an ion permeable membrane. These are immersed in an (organic) electrolyte solution and enclosed in a container. Both anode and cathode (materials) can (reversibly) alloy or intercalate lithium ions. The free energy difference between lithium intercalated in the anode and cathode is what drives the battery. LIBs achieve very high energy densities due to lithium's light mass, the high intercalation capacities of the active materials and the high operation voltage $(\sim 3.4 \mathrm{~V})^{7}$.

Both anode and cathode are typically composed of a mixture of active and conductive nanoparticles, coated on metal current collectors. Active particles are typically layered materials (such as $\mathrm{LiTiO}_{2}$ or graphite for the anode and $\mathrm{LiNi}_{x} \mathrm{Mn}_{y} \mathrm{Co}_{z} \mathrm{O}_{2}$ for the cathode) which intercalate lithium; i.e. allow it to sit between the layers. The conductive nanoparticles are superconductive carbon blacks that 'wire' the active particles to the current collectors. The particles are held together by a (polymeric) binder, which creates an open porous electrode with a high surface area. The electrodes are immersed in an electrolyte solution and are electronically separated (from shorting) by an ion permeating polymer separator membrane. The electrolyte is a solution of a lithium salt such as $\mathrm{LiPF}_{6}$ in a aprotic solvent with a high dielectric constant, usually a mixture of linear and cyclic alkyl carbonates.

A critical component of traditional LIBs is the Solid Electrolyte Interphase (SEI) layer on the anode. Most lithium ion based chemistries operate at very high voltages $(>3 \mathrm{~V})$, usually well outside the electrochemical stability window of most electrolyte solutions $\left(\sim 1-2 \mathrm{~V} \text { vs } \mathrm{Li} / \mathrm{Li}^{+}\right)^{4}$. This means that the electrolyte is prone to reduction at the anode of the battery. During the initial assembly and operation of a LIB, a small amount of electrolyte (and impurities) gets reduced, 
and the decomposition products form an electronically insulating layer on all electrode surfaces that are in contact with the electrolyte. This SEI layer forms a passivation barrier (analogous to the oxide layer on aluminum) and kinetically prevents further electrolyte decomposition. While SEI is electronically insulating, it does allow ion transfer through it and hence allows continued LIB operation at high potentials. The SEI in LIBs is complex, very dynamic and governs how a battery performs and ages on continued electrochemical cycling (charging and discharging). For example, some materials expand significantly in volume when they intercalate lithium. This causes the SEI to crack, thereby exposing electrolyte to the fresh surface, triggering additional decomposition and capacity loss.

\section{The Aqueous Redox Flow Battery}

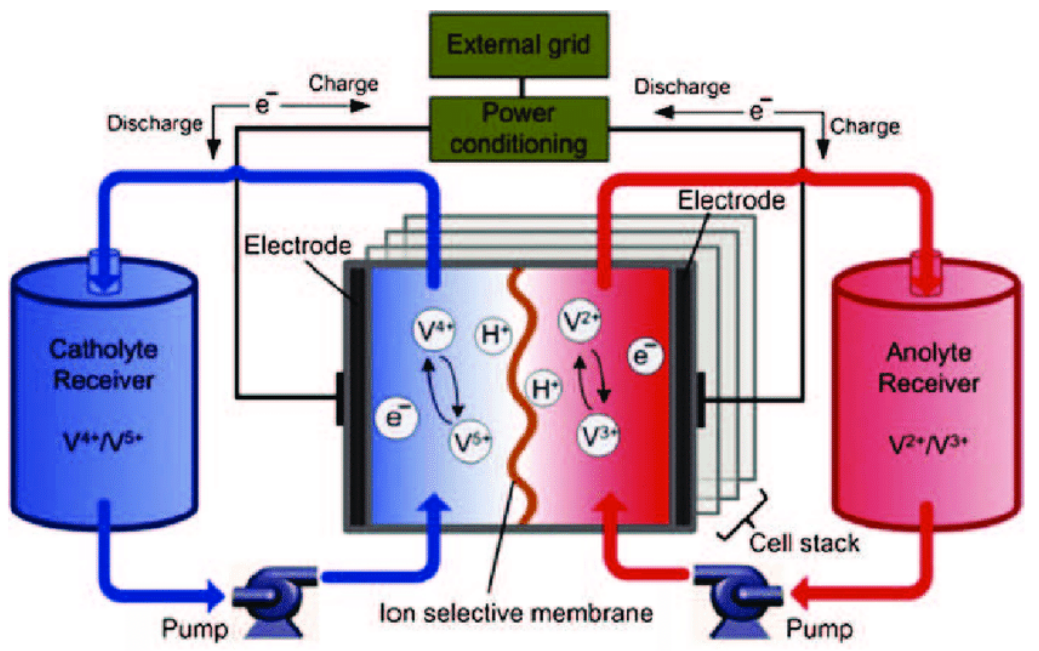

Figure 1.2: Cartoon of a redox flow battery. Reprinted from Ref. ${ }^{3}$. 
The aqueous redox flow battery (Fig.1.2) is a rechargeable electrochemical battery that decouples power generation from storage. Energy is reversibly stored in 'charged' electrolyte solutions which differ in concentration or ion oxidation state. These solutions are pumped through an electrochemical cell, where a redox reaction delivers power. Redox flow batteries have been seen as promising candidates for grid storage, with a few operational commercial systems existing in 2019. The most common type of redox flow system is the Vanadium redox flow battery ${ }^{5}$. This acidified system is composed of a catholyte with $\mathrm{V}_{2} \mathrm{O}^{+}$and $\mathrm{VO}^{2+}$ ions and an anolyte of $\mathrm{V}^{3+}$ and $\mathrm{V}^{2+}$ ions. Both electrolytes are pumped into a cell where they are separated by an ion selective membrane. In the catholyte $\mathrm{V}_{2} \mathrm{O}^{+}$is converted to $\mathrm{VO}^{2+}$ and in the anolyte $\mathrm{V}^{3+}$ into $\mathrm{V}^{2+}$, delivering power through current collectors.

A significant advantage to these aqueous redox flow batteries is their operational flexibility and relatively low material cost. Unlike traditional solid batteries, it is easy to have inline monitoring of the electrolyte 'health' and chemistry adjustments can be made during the life of the battery. A huge disadvantage however, is their very low specific energy density; an order of magnitude below lithium ion systems. ${ }^{1}$

\section{The Semi Solid Flow Battery}

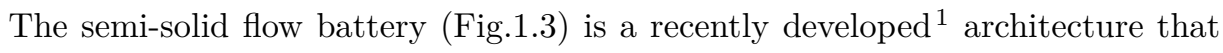
combines the flexibility of flow batteries with the high energy density of LIBs. It trades the aqueous fluid electrodes of redox flow batteries with colloidal slurries. These slurries contain a combination of active and conductive nanoparticles dispersed in an electrolyte solution. Like traditional lithium batteries, the active 


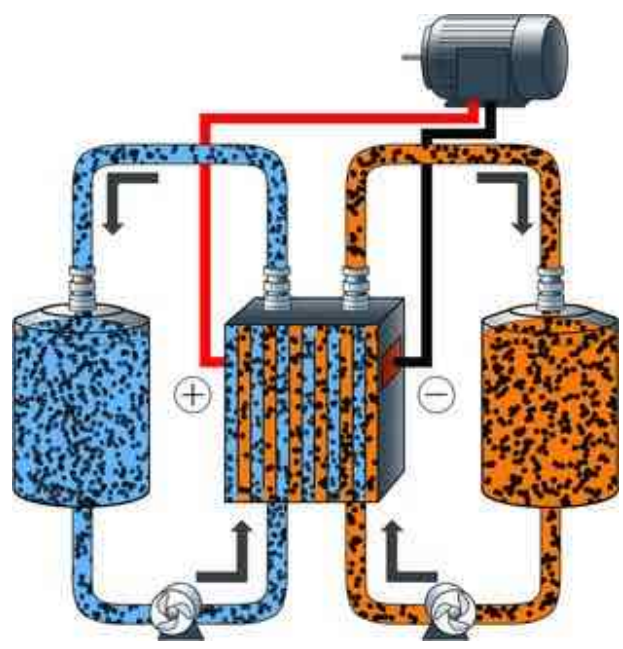

Figure 1.3: Cartoon of a semi solid flow battery. Printed with permission from John Wiley and Sons, Ref. ${ }^{1}$.

nanoparticles intercalate or alloy with lithium, while conductive nanoparticles form percolated gel networks and 'wire' the active particles to the current collectors.

\subsection{Motivation: The Move to Fluid Electrodes}

The move to slurry based fluid electrodes, while promising, presents a host of technical challenges; the foremost due to their inherent dynamic nature. Colloidal interactions between the different constituent particles now determine the fluid microstructure and consequently its properties. Mechanical stresses, as applied during pumping, operation and storage can change the microstructure. Electrochemical cycling can cause further changes.

To address these challenges, we identified 3 main questions that we try to 
answer in this thesis:

- What are the colloidal interactions of the fluid electrode particles?

- What are the effects of mechanical stresses and mechanical history?

- What are the effects of electrochemical state and history?

\subsection{Thesis Outline}

In Chapter $\mathbf{2}$ we elucidate the colloidal interactions between the conductive particles in the fluid electrodes. We study the agglomeration behaviour of dilute carbon black suspensions versus the electrolyte solution's salt concentration using dynamic light scattering. We find a concentration dependent agglomeration behaviour, with diffusion limited agglomeration at typically used salt concentrations.

Armed with this result, in Chapter 3 we study concentrated (percolated) carbon black suspensions in a home-made 'Rheoimpedance' device. With precise control of the fluid electrode's mechanical state and history, we study the effect of shear (history) on rheology and electronic conductivity. We find a novel two step agglomeration process that determines the fluid electrodes properties in a non-intuitive way. With a model, we try to extract parameters that define the microstructure in various mechanical states.

With the understanding that mechanical history control is crucial, in Chapter 4 we describe a setup to simultaneously control a fluid electrode's mechanical and electrochemical state (and history). We demonstrate the instrument on multiple real fluid electrode systems. 
Using the 'Cycling Rheoimpedance' setup, in Chapter $\mathbf{5}$ we study the effect of electrochemical cycling on a SSFB anolyte's rheology and electrochemistry. We find signs of dynamic (state-of-charge dependent) insulating SEI layer formation, where it is not expected, with drastic effects on the fluid electrode.

In Chapter 6 we directly monitor this layer formation on thin films of carbon and titania using the Quartz Crystal Microbalance. Using a viscoelastic model, we differentiate between mass changes due to intercalation and SEI formation. We find direct evidence for SEI formation on both films at operating voltages where none is expected. We also find similar SEI formation for a second electrolyte solution. 


\section{Bibliography}

[1] M. Duduta, B. Ho, V. C. Wood, P. Limthongkul, V. E. Brunini, W. C. Carter, and Y.-M. Chiang. Semi-solid lithium rechargeable flow battery. Advanced Energy Materials, 1(4):511-516, 2011.

[2] B. Dunn, H. Kamath, and J.-M. Tarascon. Electrical energy storage for the grid: a battery of choices. Science, 334(6058):928-935, 2011.

[3] X. Luo, J. Wang, M. Dooner, and J. Clarke. Overview of current development in electrical energy storage technologies and the application potential in power system operation. Applied energy, 137:511-536, 2015.

[4] A. Wang, S. Kadam, H. Li, S. Shi, and Y. Qi. Review on modeling of the anode solid electrolyte interphase (sei) for lithium-ion batteries. npj Computational Materials, 4(1):1-26, 2018.

[5] A. Z. Weber, M. M. Mench, J. P. Meyers, P. N. Ross, J. T. Gostick, and Q. Liu. Redox flow batteries: a review. Journal of Applied Electrochemistry, 41(10):1137, 2011.

[6] K. Xu. Nonaqueous liquid electrolytes for lithium-based rechargeable batteries. Chemical reviews, 104(10):4303-4418, 2004.

[7] M. Yoshio, R. J. Brodd, and A. Kozawa. Lithium-ion batteries, volume 1. Springer, 2009. 


\section{Chapter 2}

\section{Colloidal Interactions}

Yan Zhang, Aditya Narayanan, Frieder Mugele, Martien Cohen Stuart, Michel Duits

The research described in this Chapter has been published as:

Charge inversion and colloidal stability of carbon black in battery electrolyte solutions, Colloids and Surfaces A, 489:461-468, 2016

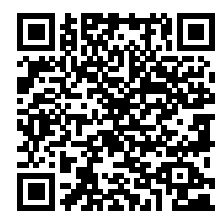




\subsection{Abstract}

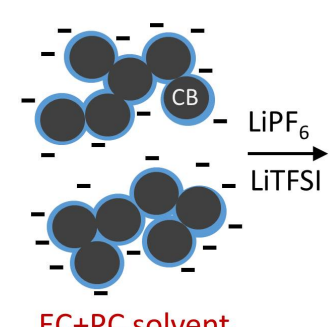

$\mathrm{EC}+\mathrm{PC}$ solvent

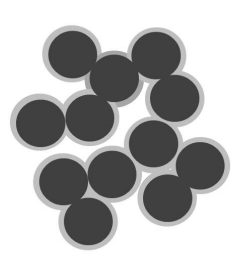

CB flocculates

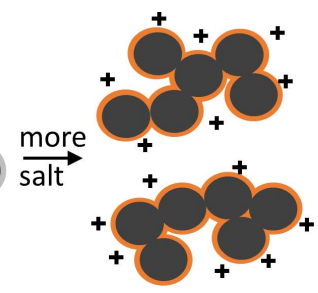

stable CB

We studied the influence of salt on a commercially available carbon black (Ketjenblack $600, \mathrm{~KB}$ ) in carbonate solvents commonly applied in rechargeable batteries. Adopting the typically used salts: lithium hexa-fluorophosphate $\left(\mathrm{LiPF}_{6}\right)$, lithium bis(trifluoromethane sulfonyl) imide (LiTFSI), as well as sodium hexafluorophosphate $\left(\mathrm{NaPF}_{6}\right)$ dissolved in mixtures of ethylene carbonate and propylene carbonate, we investigated both the zeta potential and the flocculation kinetics of the KB particles as a function of salt concentration between $0.01 \mathrm{mM}$ and 1.0 M. Clear evidence was found for the preferential adsorption of cations. In the absence of salt, KB was found to carry a negative surface charge, but this gets neutralized by $\mathrm{Li}^{+}$at very low concentrations $(\sim 1 \mathrm{mM})$, and by $\mathrm{Na}^{+}$at intermediate concentrations $(\sim 30 \mathrm{mM})$. In the case of lithium ions, the increased adsorption at higher concentration led to a recovery of the colloidal stability around 3 - $30 \mathrm{mM}$, depending on the anion. At high concentrations exceeding 30-100 mM, all salts cause flocculation of the KB particles, due to a reduction of the electric double layer thickness. Since the charge neutralization of the KB by $\mathrm{Na}^{+}$takes place in the same concentration regime, no re-entrant stability is found for $\mathrm{Na}^{+}$. These findings could have implications in formulation protocols for semi-solid flow batteries, or other systems where an intermediate stable regime 
could assist mixing and/or structure formation at small length scales.

\subsection{Introduction}

The development of sustainable energy storage systems has become an urgent issue due to the limited amount of fossil fuels / natural gas and their huge rate of consumption. Rechargeable lithium ion batteries are one of the most developed in the past few decades and commonly applied in portable electronics, electric vehicles, aerospace devices, etc. ${ }^{36}$. Flow batteries offer more flexibility than solid form batteries, but the energy density of flow batteries is relatively low ${ }^{14}$. A new type, the so-called semi-solid flow battery (SSFB), was invented by MIT and has both the advantage of flow battery's flexibility and the high energy density found in solid batteries ${ }^{10}$. SSFBs differ from other batteries in that their electrodes are dispersions or suspensions, often composed of conductive nanoparticles (CNPs, often carbon black) and electrochemically active particles (EAPs, normally metal oxides which accommodate or release lithium ions during charge and discharge) in electrolyte solutions. Electrons reacting at the redox active particles are conducted by the CNPs to and from current collectors connected to an external circuit.

Since the emergence of SSFBs in 2011, optimization of their composition (EAPs, CNPs, solvent, salt) has been explored ${ }^{31,48}$ by measuring the rheological, electrochemical and conductive properties of the fluid electrode in order to understand and improve battery performance. Here colloidal interactions play a crucial role, via their determining influence on both the structure and the dynamics of the fluid. The CNPs, in spite of being present at much lower volume 
fractions than the EAPs, often dominate both the conductive and rheological behavior of the entire fluid via the formation of a flocculated network. Besides that, the colloidal interactions between active and conductive particles can influence the battery performance ${ }^{6,49}$. However, the situation at present is that only little is known (and much is left to optimize) about the structure and strength of the (EAP surrounded) CNP network, an important reason being that the colloidal interactions between the CNPs are yet to be fully understood.

Both the specific solvent and the used salts (the electrolyte solutions) in SSFBs are the reason for this lack of knowledge. Similar to classical lithium batteries, the electrolytes in SSFBs are typically solutions of Li or $\mathrm{Na}$ salts dissolved in carbonate solvents. For example, $\mathrm{Li}(/ \mathrm{Na}) \mathrm{PF}_{6}, \mathrm{Li}(/ \mathrm{Na}) \mathrm{TFSI}, \mathrm{Li}(/ \mathrm{Na}) \mathrm{BF}_{4}$, $\mathrm{Li}(/ \mathrm{Na}) \mathrm{ClO}_{4}$ are used as salts, while ethylene carbonate (EC), propylene carbonate (PC), dimethyl carbonate (DMC) and diethyl carbonate (DEC) are used as solvents. Various combinations of these salts and solvents result in different electrochemical properties ${ }^{7,34,44}$.

In more common solvents such as water, ethanol, hydrocarbons, etc., the behavior of Carbon Black has been better studied. Since van der Waals attractions between CBs are ubiquitous, surface oxidation followed by surface modification generally has to be applied in order to disperse carbon black ${ }^{11,12,27}$. In aqueous systems, the surface charge likely originates from carboxylate groups, while the extent of screening can be controlled via the salt concentrations. In non-polar solvents there is little screening of the electrostatic interactions, while the surface potential can vary per system (the origin is not always clear). In the battery community, the mixture of EC and PC is recognized as one of the best performing solvents ${ }^{33,44}$. Both EC and PC cannot be ranked amongst conventional solvents, 
since they are aprotic while having a high dielectric constant. The latter allows dissociation of certain salts up to high concentrations (as needed for achieving high conductivity). In turn, the dissociated ions can change both the surface potential and the electric double layer thickness. This could mean that the electrostatic interactions bear similarity to those in aqueous systems. However, even in that case there might be practical differences in the behavior at low salt concentrations, because the sensitivity of carbonate solvents to impurities is quite high.

A particular process that could modify the electrostatic particle interactions, is specific ion adsorption. Depending on the signs of the native surface charge and the adsorbing ion, even reversal of the surface charge is possible. This phenomenon has been studied for a variety of colloids $4,5,20,25,32,38$, including many oxides in aqueous environments ${ }^{4,20,25}$. Sign reversal of the zeta potential induced by alkali metal cations such as $\mathrm{Li}^{+}$and $\mathrm{Na}^{+}$has been found in a few cases (e.g. ${ }^{24}$ ), while for protons it is a well-known phenomenon, also in non-aqueous solvents ${ }^{4}$. In this paper we focus on the principal aspects of the colloidal stability of Carbon Black suspensions in electrolyte solutions containing the same ingredients as in a typical SSFB. We chose three electrolyte solutions, namely LiTFSI, $\mathrm{LiPF}_{6}$ and $\mathrm{NaPF}_{6}$ dissolved in a 1:1 binary mixture of EC and PC. Measurements of the zeta potential as a function of salt concentration are combined with determinations of the time-dependent average hydrodynamic (aggregate) diameter with Dynamic Light Scattering (DLS). By starting the DLS experiments with the suspensions in a non-flocculated state, we measure the flocculation rate, and calculate the stability ratio. Combining the observations from the two types of experiments allows us to draw conclusions about the ion adsorption and stabilization mechanism. 


\subsection{Theory}

In this section we summarize the approach and key equations as used to measure the stability ratio of the KB particles, as a function of salt concentration. The initial stage of the flocculation of particles with uniform size and shape can be described via the second-order reaction:

$$
\frac{d N}{d t}=-k N^{2}
$$

where $N$ is the concentration of the non-flocculated particles and $k$ is the flocculation rate constant. Early stages of the flocculation process can be analyzed by measuring the average hydrodynamic radius $\left(R_{h}\right)$ with DLS as a function of time ${ }^{21}$. The linear dependence of $R_{h}$ on time in this regime allows to determine $d R_{h} / d t$, which is proportional to the flocculation rate. The average hydrodynamic radius is calculated using the Stokes-Einstein equation:

$$
D=\frac{k_{b} T}{6 \pi \eta R_{h}}
$$

Where $D$ is the measured diffusion coefficient, $\eta$ the viscosity of the medium, $k_{b}$ the Boltzmann constant and $T$ the absolute temperature. Unlike many aqueous salt solutions, the viscosity of solutions in carbonate solvent depends significantly on the salt concentration: it nearly triples when the concentration of LiTFSI ${ }^{46}$, $\mathrm{LiPF}_{6}{ }^{22}$ and $\mathrm{NaPF}_{6}{ }^{2}$ reaches $1 \mathrm{M}$ (see also Appendix). This is of importance when calculating $R_{h}$, but also for the proper normalization of the flocculation rate: in more viscous media, the diffusion-limited flocculation of particles takes longer. This aspect, expressed by the factor $k_{S m o l}=8 k_{b} T / 3 \eta$ in the Smoluchowski equation is taken into account by multiplying $\eta_{r}$ and $d R_{h} / d t$, where $\left(\eta_{r}=\eta_{s a} / \eta_{s o}\right.$ 
with $\eta_{s a}$ the viscosity of the salt solution and $\eta_{s o}$ likewise for the solvent). The stability ratio 35,40 is then defined as:

$$
W=\frac{\left[d\left(R_{h} \eta_{r}\right) / d t\right]_{\max }}{d\left(R_{h} \eta_{r}\right) / d t}
$$

This quantity equals 1 (regardless of the viscosity) in all cases of unhindered flocculation. Repulsive interactions give rise to an energy barrier, which slows down the flocculation and hence increases W. In the limit where the particles do not flocculate at all, $W$ approaches infinity. We remark that the use of $d R_{h} / d t$ to calculate the stability ratio relies on the assumption that $R_{h}$ increases linearly with time, which can only be expected in the early stages of the flocculation ${ }^{42}$. This implies that the analysis should be performed well within the so-called halftime, in which the number of entities is reduced by a factor of two:

$$
t_{1 / 2}=\frac{3 \eta}{4 k_{b} T N_{0}}
$$

\subsection{Experiments}

\subsubsection{Materials and sample preparation}

Carbon black (Ketjenblack 600JDP) (KB) was obtained from AkzoNobel (the Netherlands) and dried in a vacuum oven for 24 hours before use. Ethylene carbonate (EC) (anhydrous 99\%+ purity, $<0.005 \%$ water) and propylene carbonate (PC) (anhydrous $99.7 \%+$ purity, $<0.002 \%$ water) were obtained from Sigma Aldrich (the Netherlands) and used as received. The binary mixture of EC and PC was 1:1 by volume (henceforth referred to as EC PC 1:1). LiTFSI 
(99\% purity) was obtained as a gift from Solvionic (France). $\mathrm{LiPF}_{6}$ and $\mathrm{NaPF}_{6}$ $(99 \%+$ purity) were both purchased from Alfa Aesar (the Netherlands). All salts were used as received. Samples for SEM imaging were prepared by spreading a paste-like mixture of KB in ethanol on cleaned silicon wafers followed by drying in ambient air for half an hour. KB stock suspensions $(0.01 \% \mathrm{wt})$ were prepared by mixing the powder with EC PC 1:1 in an MBraun Argon-filled glove box $\left(\mathrm{O}_{2}\right.$, $\mathrm{H}_{2} \mathrm{O}<0.5 \mathrm{ppm}$ ), followed by ultrasonic treatment for 15 minutes. Samples for DLS and zeta potential measurements were prepared by initially mixing the required amount of the stock dispersion with EC PC 1:1 in polystyrene cuvettes; they were then removed from the glove box and sonicated for 15 minutes. Finally, salt solution (the concentration varied from $1.5 \times 10^{-4} \mathrm{M}$ to $1.5 \mathrm{M}$ ) was added. The mixtures were then allowed to equilibrate for at least 24 hours and sonicated for another 15 minutes before the experiments. DLS and zeta potential measurements could only be performed outside the glovebox.

\subsubsection{Measurement of hydrodynamic radius and zeta potential}

Hydrodynamic radii of the KB aggregates were measured as a function of time in a Malvern Nano ZS zetasizer using sealed polystyrene containers. Immediately prior to each experiment, the sample was sonicated for 5 minutes to obtain a well-defined, non flocculated starting state. Considering that the start -up time of the DLS measurement amounts $\sim 2$ minutes, and that about 30 minutes are needed for a reliable measurement of $d R_{h} / d t$, we have aimed $t_{1 / 2}$ to be $\sim 1$ hour. According to Eq. 2.4, $N_{0}$ should then be $\approx 3 \times 10^{14}$ particles $\mathrm{m}^{-3}$. Since our unit particles are sintered (i.e. permanent) aggregates with complicated fractal-like 
shapes (see below), the quantitative relation between the theoretical $N_{0}$ and the experimental weight fraction $w$ is not known. In the expression:

$$
w=\frac{N_{0} v_{p} \rho_{p}}{\rho_{s}}
$$

with $v_{p}$ the solid volume of a unit particle, and $\rho_{p}, \rho_{s}$ the mass densities of $\mathrm{KB}$ and solvent (respectively $\approx 1.8$ and $1.25 \mathrm{~g} / \mathrm{mL}$ ), $v_{p}$ is unknown. Measurement of the hydrodynamic radius with DLS gave $R_{h} \approx 350 \mathrm{~nm}$. Assuming that the contour of the fractal-like particle is a sphere with this radius, and estimating the internal filling volume fraction to be $\approx 0.06$, we obtain $\mathrm{w}=1.3 \times 10^{-6}$. Using this mass fraction in the preparation of the suspensions, we obtained a good linearity in the data of $R_{h}$ vs time (see Fig. 2.4). Zeta potentials were measured in the same zetasizer using a solvent-resistant dip cell. Samples were transferred into the cell inside the glovebox, sealed during transportation to the Zetasizer, and measured under Nitrogen protection. A thermal equlibration time of 120 $\mathrm{s}$ at $25 \mathrm{C}$ was allowed before starting the measurements. The measurement voltage was always in the range of 4.5 to $20 \mathrm{~V}$. The instrument calculates the electrophoretic mobility $U_{E}$ from the average particle velocity and the electric field strength, where the latter is calculated from the measured ion current and the conductivity of the continuous phase. The zeta potential $\zeta$ is then calculated from the electrophoretic mobility, using an appropriate model. We take the simplest equation from Smoluchowski:

$$
U_{E}=\frac{\epsilon \zeta}{\eta}
$$

with $\epsilon$ the dielectric constant of the continuous phase ${ }^{9}$, for which we have taken 
that of the solvent. Considering the aforementioned complicated particle shape, we refrain from taking electrophoretic retardation and relaxation corrections into account; values reported here are therefore to be considered as estimates rather than precise determinations.

\subsection{Results and Discussion}

\subsubsection{KB in pure EC PC solvent}

Carbon black contains 90-99\% carbon; this makes it strongly hydrophobic ${ }^{26}$ and hardly dispersible in water. Generally, CB in suspension exist as units of chemically connected nanoparticles. Particle sizes have been reported to be $794 \mathrm{~nm}$ when suspended in water, and $181 \mathrm{~nm}$ in ethanol, in both cases much larger than the size of subunits that can be distinguished in electron micrographs ${ }^{45}$. Surface modifications such as oxidation introduce carboxylic groups and significantly improve the colloidal stability in both aqueous and non-aqueous solvents ${ }^{19}$. The adsorption of surfactants such as poly (oxometalate) is also effective to disperse carbon black in water, as aggregates smaller than $100 \mathrm{~nm}^{13}$.

Some key properties of the Ketjenblack particles used in our experiments are summarized in Table 2.1, and are further discussed below. SEM images of (Figure 2.1) show structural subunits with diameters of around 20-30 nm. In contrast, the hydrodynamic radius as measured by DLS was $350 \pm 88 \mathrm{~nm}$. This clearly indicates that in EC PC 1:1 (without added salt), KB exists as structures that are much larger than the 20-30 nm units. To examine the nature of connections between the small building blocks, we subjected the suspensions to dilution (down to the detection limit of the DLS) and ultrasonication (for at least 1 hour). In either 
experiment, no significant changes in were observed. Additional tests in which the particles were left to settle due to gravity (a behaviour that was manifested in the flocculated systems) did not shown any signs of sedimentation, even after several days.

Each of these observations indicates that $\mathrm{KB}$ is colloidally stable in EC PC 1:1 (without added salt). The zeta potential of KB, measured to be $\sim 47 \mathrm{mV}$, shows that the particles carry a significant negative surface charge, which is likely responsible for the colloidal stability. The fact that the overall hydrodynamic radius is much larger than that of the $20-30 \mathrm{~nm}$ structures indicates that the latter are chemically bound (rather than flocculated). No further effort was taken to disperse $\mathrm{KB}$ into smaller sizes; the fractal-like $350 \mathrm{~nm}$ particles were treated as permanent and inseparable in this particular solvent.

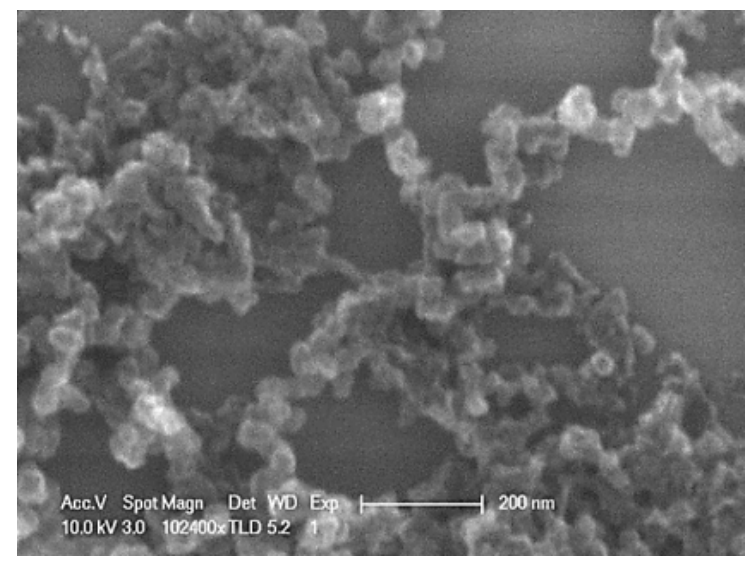

Figure 2.1: SEM image of Ketjenblack.

The origin of surface charge for particles suspended in a liquid can be protonation or deprotonation of functional surface groups (typically hydroxyl) ${ }^{28}$, adsorption of ions from solution ${ }^{17}$, solvation and release of the ions from the 


\begin{tabular}{cccc} 
& $R_{S E M}(\mathrm{~nm})$ & $R_{h}(\mathrm{~nm})$ & $\zeta(\mathrm{nm})$ \\
\hline $\mathrm{KB}$ & $\sim 15$ & $350 \pm 88$ & -47
\end{tabular}

Table 2.1: Particle radius (from $S E M$ and $D L S$ ) and zeta potential of $K B$ in EC PC 1:1 in absence of salt.

particle $^{20}$, or a combination of these ${ }^{18,37}$. Which mechanism(s) is(are) applicable depends on the particle surface chemistry, whether the solvent is protic or aprotic, the dielectric permittivity of the solvent (affecting the solvation of ions), etc. In case of particles in non-aqueous solvents with a low dielectric constant, it is generally less clear where the surface charge comes from. Impurity ions and moisture could play a role ${ }^{29}$. For carbon blacks, the origin of the surface charge is even more complex, due to its source or batch dependent surface chemistry. For example, carbon atoms may bond with hydrogen and oxygen containing groups, such as quinone, ether, aldehyde and phenol, which act as defects. The zeta potential of carbon nanotubes depends on the dielectric constant of the dispersing medium, and varies in both value and sign because electron transfer occurs at different defect sites ${ }^{30}$; this may apply to CB as well. Furthermore, Kosmulski ${ }^{24}$ and $\mathrm{Xu}$ et $\mathrm{al}^{45}$ ascribed the surface charge and potential in non-aqueous solvents to an electron transfer between the particles and the solvent.

Specific for our system is that EC and PC have high dielectric constant (EC: 90; PC: 64) which could allow protons of the carboxylic groups on the CB surface to dissociate ${ }^{12}$. It is also possible for the oxygen lone pairs in EC and PC to transfer to the carbon black surface. We speculate that the negative surface charge of $\mathrm{KB}$ in EC:PC comes from a combination of these two mechanisms. 


\subsubsection{KB in electrolyte solutions}

\section{Ion dissociation}

Anticipating that the colloidal stability of KB will be intimately related to the presence of ions, we first examine the relation between the amount of dissolved (1:1) salt per unit volume (c), and the actual free ion concentrations. There are two extreme regimes where these two quantities may differ significantly. At the high concentrations corresponding to SSFBs, the salts may not be completely dissociated anymore, while at very low concentrations, (trace) impurities from the materials themselves or from the environment may significantly contribute to ionic composition of the liquid.

Measurements of the electric conductivity provide a suitable way to examine both regimes. They were performed with the Zetasizer as part of the determination of the zeta potential of the KB particles. To assess whether in these measurements also the KB could contribute to the conductivity, we compared the conductivities the salt-free solvent with and without KB. Both were measured to be $1.7 \pm 0.1 \mu \mathrm{S} / \mathrm{cm}$, indicating that the contribution of $\mathrm{KB}$ is indeed negligible.

\section{Equivalent conductivities:}

Figure 2.2 shows for each of the three salts, how the apparent molar conductivities (conductivity due to added salt, divided by the concentration) depend on the salt concentration. For concentrations roughly between 0.1 and $100 \mathrm{mM}$, the (normalized) conductivities are constant per salt, indicating that the salts are completely dissociated, and contributions of other components than the salt alone, are negligible.

In the low salt concentration regime below $0.2 \mathrm{mM}$, the apparent molar con- 


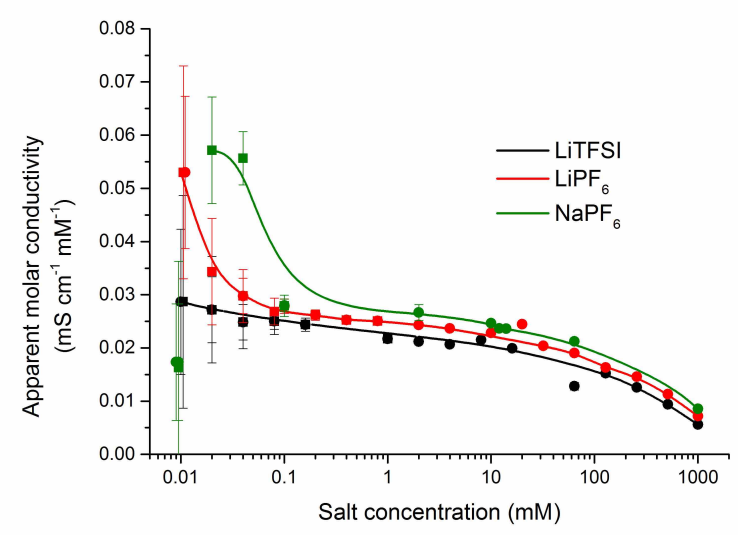

Figure 2.2: Apparent molar conductivity of LiTFSI, $\mathrm{LiPF}_{6}$ and $\mathrm{NaPF}_{6}$ versus concentration of salt. The background conductivity of the solvent was subtracted; For $c<0.1 \mathrm{mM}$, large error bars result after the subtraction and normalization. For $c=0.01 \mathrm{mM}$ some data points are shifted horizontally to allow distinction between different experiments

ductivities fall slightly above the values expected for the amount of dissolved salt. This could mean that (for the $\mathrm{PF}_{6}$ salts) some additional ions were via impurities. Assuming a specific conductivity comparable to $\mathrm{HCl}$, Kortschot et $\mathrm{al}^{3,23}$, found impurity concentrations of around $0.02 \mathrm{mM}$.

Water, as present in the starting materials, or as taken up from the ambient atmosphere might be a source of impurities. According to manufacturer, the water content of a EC-PC mixture should be $\leq 35 \mathrm{ppm}$, while other investigators have measured it to be $<20 \mathrm{ppm}^{47}$. Moisture in the system could change the surface potential of particles dispersed in nonaqueous systems ${ }^{24}$. Furthermore, $\mathrm{LiPF}_{6}$ and $\mathrm{NaPF}_{6}$ are known to react with water to produce $\mathrm{HF}$ and $\mathrm{F}^{-1,8}$ while LiTFSI is rather stable. The relatively high equivalent conductivity of 
$\mathrm{Li}(/ \mathrm{Na}) \mathrm{PF}_{6}$ at $<0.1 \mathrm{mM}$ salt compared with that of LiTFSI might thus be attributed to water. However, experiments aimed at corroborating this hypothesis by adding water, turned out inconclusive.

We now turn to the regime of high salt concentrations. Both EC and PC have a high dielectric constant, which favours ionization. However for c $>10 \mathrm{mM}$, it is indicated from the conductivities that ion pair formation becomes significant. In literature, the degree of dissociation ( has been estimated to be 0.67 for 0.6 M LiTFSI ${ }^{16}, 0.67$ for $1.0 \mathrm{M} \mathrm{LiPF}_{6}{ }^{15}$ and 0.34 for $0.7 \mathrm{M} \mathrm{NaPF}_{6}{ }^{2}$ in EC PC 1:1. Inserting these numbers into the expression for the dissociation constant $(K)$ :

$$
K=\frac{\alpha^{2} c}{1-\alpha}
$$

and calculating the actual ion concentrations as a function of the concentration of added salt, we find that the dissociation is almost complete below $10 \mathrm{mM}$ for each of the salts, while for higher concentrations the degree of dissociation (at the same c) follows the order: $\mathrm{NaPF}_{6}<\mathrm{LiTFSI}<\mathrm{LiPF}_{6}$. We remark that the equivalent conductivities in Fig.2.2 do not follow this trend; suggesting that in EC:PC the mobility of $\mathrm{Na}^{+}$is higher than that of $\mathrm{Li}^{+}$.

\section{Debye lengths:}

From the measurements in Fig.2.2 and and the assumed specific conductivities, the Debye length $\kappa^{-1}$ can be calculated. In the calculations we included the both the added ions and impurities in the solvent. The concentration of the latter was estimated from a comparison with the conductivity of pure solvent, similar to $\left.^{3,23}\right)$. Possible ion concentration changes due to the reaction with moisture were not taken into consideration. In Figure 2.3 we plot it against $1 / \sqrt{c}$ for the three electrolyte solutions. 


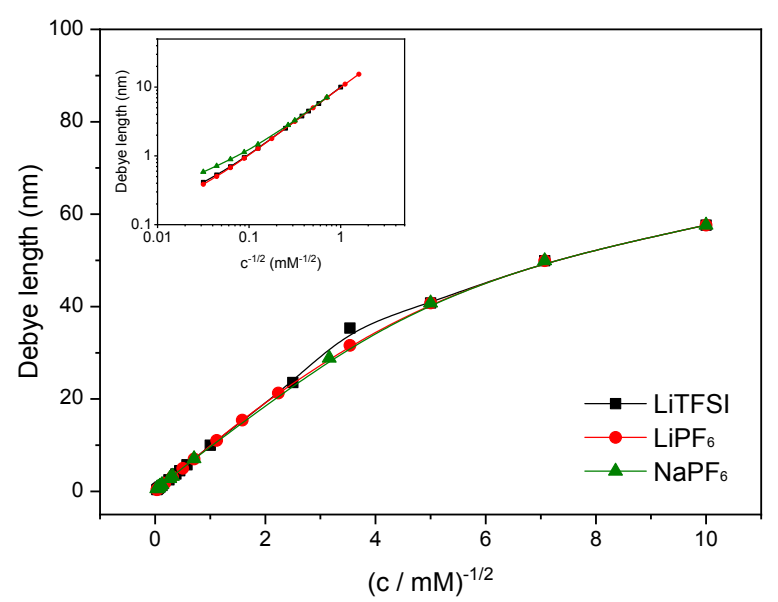

Figure 2.3: Debye length against the inverse of the square root of the salt concentration for LiTFSI, LiPF 6 and NaPF . Solid lines are drawn to guide the eye. Inset shows the same data in a log-log plot.

In case of complete dissociation and absence of impurities, this plot should show a straight line with a slope of $\sim 10 \mathrm{mM}^{0.5} \mathrm{~nm}$. This appears to be the case for concentrations in the range $0.25-5 \mathrm{mM}$. At lower concentrations (higher $c^{-1 / 2}$ ), the effects of the impurities show up, while at higher concentrations the effect of incomplete dissociation becomes visible as an upward curvature (see the $\log -\log$ plot in the inset).

Clearly, the thickness of the electric double layer undergoes strong variations as the salt concentration is varied between $10 \mu \mathrm{M}$ and $1 \mathrm{M}$, reaching the $\mathrm{nm}$ range (where van der Waals interactions play a role) at concentrations around $0.1 \mathrm{M}$ (similar to aqueous systems). A calculation of (assuming the aggregate radius to be relevant) reveals that (i.e. relatively thin double layers) for all salt concentrations investigated. This justifies the calculation of zeta potentials 
using the Smoluchowski equation (as was done in Sec.2.3). Another observation from Fig.2.3 is that the variations in Debye length between the different salts at the same concentration, are generally minor; suggesting that any differences in colloidal stability of $\mathrm{KB}$ in presence of the different salts are not be related to differences in screening.

\section{Zeta potential and colloidal stability of KB}

Figure 2.4 shows some typical curves of the time-dependent hydrodynamic radius of KB particles in solutions of EC PC 1:1 containing different amounts of salt (in this case LiTFSI). In this graph $R_{h}$ is multiplied with the relative viscosity of the salt solution (compared to salt-free solvent), to allow direct comparison of the slopes representing the flocculation rates (see Sec. 2). The concentrationdependent viscosities of the different salt solutions are given in the Appendix. The $R_{h}$ values themselves are all close to $350 \mathrm{~nm}$ at the beginning of the experiment; this confirms that all experiments were started with non-flocculated particles. Table 2.2 lists the numerical data for all flocculation experiments with LiTFSI, together with estimated errors.

It can be concluded from Fig. 2.4 and Table 2.2 that different regions of colloidal stability of KB are found on varying the concentration of the LiTFSI salt. At very low salt concentration $(\leq 0.1 \mathrm{mM})$ the size of the $\mathrm{KB}$ particles remained constant, indicating that the suspensions were colloidally stable. Between 0.1 and $10 \mathrm{mM}$ LiTFSI flocculation occurred, corresponding to a first critical flocculation concentration (CFC). However on the addition of more salt, colloidal stability was recovered, as evidenced by a constant $R_{h}$ at concentrations between 10 and 100 $\mathrm{mM}$. Visual observation of samples in this regime did not show any significant 


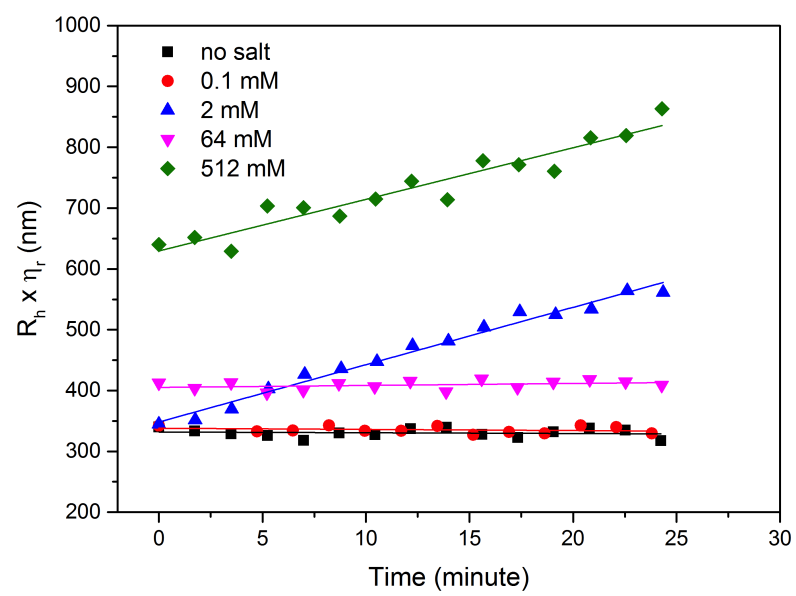

Figure 2.4: Hydrodynamic radii multiplied by the relative viscosity for $K B$ as a function of time at different concentrations of LiTFSI. The lines are the linear fits.

sedimentation, even after weeks, thus confirming colloidal stability.

To allow a closer inspection and enable a mechanistic explanation, we now consider the stability ratio in conjunction with zeta potential. Figure 2.5a shows these data for KB in solutions of LiTFSI. A very large change in zeta potential is observed, from $-47 \mathrm{mV}$ in absence of salt (not shown) to $+28 \mathrm{mV}$ at $10 \mathrm{mM}$ LiTFSI. Inversion of the sign of the zeta potential takes place between 1 and 10 $\mathrm{mM}$, while the loss of colloidal stability occurs between 0.1 and $1 \mathrm{mM}$, where the zeta potential is already close to zero $(-15 \mathrm{mV})$. These observations appear to be consistent with each other. The change in zeta potential is clearly caused by (preferred) adsorption of cations, in this case (predominantly) $\mathrm{Li}^{+}$. Increasing the salt concentration beyond $10 \mathrm{mM}$ causes the zeta potential to gradually decrease again, leading to a second loss of colloidal stability and hence a second CFC at 


\begin{tabular}{|c|c|c|c|}
\hline $\begin{array}{l}\text { Concentration of } \\
\text { LiTFSI }(\mathrm{mM})\end{array}$ & $\begin{array}{l}\text { Viscosity } \\
\text { (mPa.s) }\end{array}$ & $\begin{array}{ll}\begin{array}{l}\text { Normalized } \\
\text { culation }\end{array} & \text { rloc- } \\
(\mathrm{nm} / \mathrm{min}) & \\
\end{array}$ & Stability \\
\hline 0.16 & 2.32 & $0.58 \pm 0.20$ & Stable \\
\hline 1 & 2.32 & $6.29 \pm 0.21$ & Unstable \\
\hline 2 & 2.33 & $7.85 \pm 0.30$ & Unstable \\
\hline 4 & 2.33 & $6.95 \pm 0.14$ & Unstable \\
\hline 8 & 2.35 & $6.92 \pm 0.20$ & Unstable \\
\hline 16 & 2.38 & $1.03 \pm 0.28$ & Unstable \\
\hline 64 & 2.53 & $0.22 \pm 0.28$ & Stable \\
\hline 128 & 2.75 & $0.81 \pm 0.19$ & Stable \\
\hline 256 & 3.21 & $1.36 \pm 0.51$ & Unstable \\
\hline 512 & 4.21 & $6.01 \pm 0.57$ & Unstable \\
\hline 1000 & 7.33 & $6.89 \pm 0.84$ & Unstable \\
\hline
\end{tabular}

Table 2.2: Particle radius (from SEM and DLS) and zeta potential of $K B$ in EC PC 1:1 in absence of salt.

around $100 \mathrm{mM}$. The zeta potential is found to be $($ now +$) 15 \mathrm{mV}$ in this regime. The reason for the second flocculation is the strong electrostatic screening; the Debye length is only $\approx 1 \mathrm{~nm}$ (see inset of Fig. 2.3). A CFC of around $100 \mathrm{mM}$ is a typical value for solvents of high dielectric constant; many aqueous systems have similar CFCs for monovalent salts.

It is interesting to compare the findings for LiTFSI with those for $\mathrm{LiPF}_{6}$. If the adsorption of cations is the main reason for charge inversion and re-entrant colloidal stability, similar findings are expected for the two salts. Fig. 2.5b suggests that this is indeed the case. However the salt concentrations where the transitions occur, appear to be somewhat smaller for $\mathrm{LiPF}_{6}$. Additionally, at the same salt concentration, the zeta potentials of $\mathrm{KB}$ were higher in the case of $\mathrm{LiPF}_{6}$ as compared to LiTFSI . Both differences might be due to the creation of protons, through the reaction of $\mathrm{PF}_{6}$ anions with trace water. Due to their small size, protons can be expected to adsorb relatively easily as compared to larger 


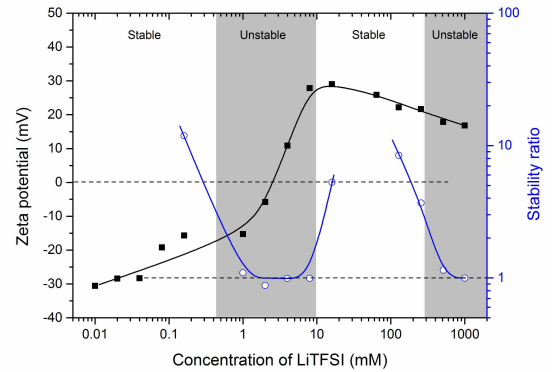

(a)

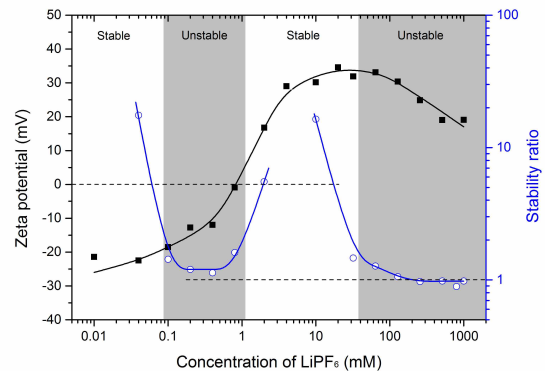

(b)

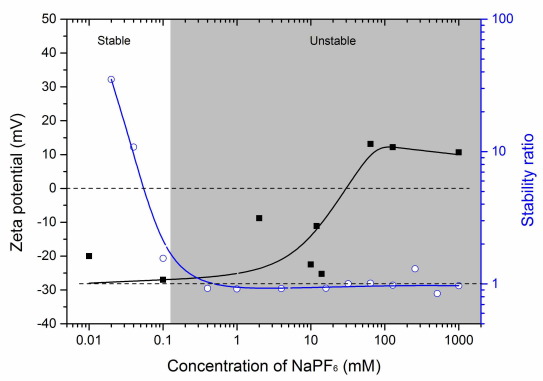

(c)

Figure 2.5: Stability ratio (open blue circles) and zeta potential (filled black squares) of KB as a function of (a) LiTFSI, (b) $\mathrm{LiPF}_{6}$ and (c) NaPF 6 concentration. Solid lines are drawn to guide the eye.

ions. Moisture has also been reported to cause charge reversal of silica particles in non-aqueous suspensions ${ }^{39}$.

Interestingly, a similar sign reversal of zeta potential from negative to positive as that reported in Fig. 2.5 was induced by alkali metal cations in other nonaqueous solvents like alcohols and dioxane for silica, titania, and other materials ${ }^{25}$. Remarkably, the sign reversal was observed at relatively low concentrations of cesium and potassium, while lithium and sodium salts were less efficient. Recent 
papers ${ }^{41,43}$ have addressed intercalation of several alkali metals into carbon via DFT calculations.

The measurements on $\mathrm{NaPF}_{6}$ solutions allow another comparison with the case of LiPF6. The data shown Figure 2.5c indicate that remarkable differences exist. Although charge (and zeta potential) inversion also occurs with $\mathrm{NaPF}_{6}$, a significantly larger amount of salt is needed, suggesting that the tendency for $\mathrm{Na}^{+}$ to adsorb onto $\mathrm{KB}$, is weaker. Additionally the finding that the zeta potentials are generally lower for the NaPF6 case is consistent. The reason for this weaker adsorption may be attributed to the much larger ion radius of $\mathrm{Na}^{+}(0.19 \mathrm{~nm})$ as compared to $\mathrm{Li}^{+}(0.07 \mathrm{~nm})$. Remarkably, the re-entrant colloidal stability found for $\mathrm{LiPF}_{6}$ is not observed with $\mathrm{NaPF}_{6}$. As the charge inversion for $\mathrm{NaPF}_{6}$ occurs at much higher salt concentration, screening due to the additional salt prevents re-stabilization in this regime.

As a final comment we briefly report a series of zeta potential measurements with $\mathrm{NaPF}_{6}$, in which less strict precautions were taken, i.e. using the dip cell in open air (rather than an atmosphere of nitrogen). In this case, where the samples become more easily contaminated, the zeta potential was found to be more or less stable at $-10 \mathrm{mV}$ for $\mathrm{NaPF}_{6}$ concentrations below $1 \mathrm{mM}$. This less negative potential (as compared to -20 to $-30 \mathrm{mV}$ in Fig. 2.5c) could suggest that proton adsorption play a role. The fact that now only $\sim 1 \mathrm{mM} \mathrm{NaPF}_{6}$ was needed to achieve charge inversion, would be consistent with that. 


\subsection{Conclusions}

Carbon black particles carry negative a surface charge and form colloidally stable suspensions in the binary solvent of ethylene carbonate and propylene carbonate. The addition of salt neutralizes the negative surface charge and causes flocculation of particles, followed by charge inversion at higher salt concentrations, due to the further adsorption of cations (lithium or sodium ions). For some systems this gives rise to a re-entrant stability at salt concentrations around $10 \mathrm{mM}$. The salt concentration where charge inversion occurs is around $1 \mathrm{mM}$ for both $\mathrm{LiPF}_{6}$ and LiTFSI although there are minor differences due to specific cation-anion interactions. Sodium ions show a much lower adsorption affinity to our carbon black, causing the $\mathrm{NaPF}_{6}$ concentration needed for charge inversion to be much higher (10-100 mM). Related to the stronger electrostatic screening, a re-entrant stability is not found for the sodium salt. Similar patterns of re-entrant stability occur in aqueous systems containing specifically adsorbed (cat)ions. 


\section{Bibliography}

[1] C. G. Barlow. Reaction of water with hexafluorophosphates and with li bis(perfluoroethylsulfonyl)imide salt. Electrochemical and Solid State Letters, 2(8):362-364, 1999.

[2] H. Bauman, J. Chilton, and A. Hultquist. Lithium anode limited cycle battery investigation. Technical report, LOCKHEED MISSILES AND SPACE CO INC PALO ALTO CA MATERIALS SCIENCES LAB, 1966.

[3] V. S. Bhat and A. K. Srivastava. Ionic conductivity in binary solvent mixtures. 5 . behavior of selected 1: 1 electrolytes in ethylene carbonate plus water at 25 degrees c. Journal of Chemical and Engineering Data, 46(5):1215$1221,2001$.

[4] N. Boon and R. van Roij. Charge reversal of moisturous porous silica colloids by take-up of protons. Journal of Colloid and Interface Science, 385:66-72, 2012.

[5] C. Calero, J. Faraudo, and D. Bastos-Gonzalez. Interaction of monovalent ions with hydrophobic and hydrophilic colloids: Charge inversion and ionic specificity. Journal of the American Chemical Society, 133(38):15025-15035, 2011.

[6] M. Cerbelaud, B. Lestriez, R. Ferrando, A. Videcoq, M. Richard-Plouet, M. T. Caldes, and D. Guyomard. Numerical and experimental study of suspensions containing carbon blacks used as conductive additives in composite electrodes for lithium batteries. Langmuir, 30(10):2660-2669, 2014.

[7] L. G. Chagas, D. Buchholz, L. Wu, B. Vortmann, and S. Passerini. Unexpected performance of layered sodium-ion cathode material in ionic liquidbased electrolyte. Journal of Power Sources, 247(0):377-383, 2014.

[8] Z. Chen, Y. Ren, A. N. Jansen, C.-k. Lin, W. Weng, and K. Amine. New class of nonaqueous electrolytes for long-life and safe lithium-ion batteries. Nat Commun, 4:1513, 2013.

[9] A. V. Delgado, F. González-Caballero, R. Hunter, L. K. Koopal, and J. Lyklema. Measurement and interpretation of electrokinetic phenomena (iupac technical report). Pure and Applied Chemistry, 77(10):1753-1805, 2005.

[10] M. Duduta, B. Ho, V. C. Wood, P. Limthongkul, V. E. Brunini, W. C. 
Carter, and Y.-M. Chiang. Semi-solid lithium rechargeable flow battery. Advanced Energy Materials, 1(4):511-516, 2011.

[11] C. Eisermann, C. Damm, B. Winzer, and W. Peukert. Stabilization of carbon black particles with cetyltrimethylammoniumbromide in aqueous media. Powder Technology, 253:338-346, 2014.

[12] M. M. Gacek and J. C. Berg. The role of acid-base effects on particle charging in apolar media. Advances in Colloid and Interface Science, 220(0):108123, 2015.

[13] P. Garrigue, M.-H. Delville, C. Labrugere, E. Cloutet, P. J. Kulesza, J. P. Morand, and A. Kuhn. Top-down approach for the preparation of colloidal carbon nanoparticles. Chem. Mater., 16(16):2984-2986, 2004.

[14] S. Hamelet, T. Tzedakis, J.-B. Leriche, S. Sailler, D. Larcher, P.-L. Taberna, P. Simon, and J.-M. Tarascon. Non-aqueous li-based redox flow batteries. Journal of The Electrochemical Society, 159(8):A1360-A1367, 2012.

[15] K. Hayamizu. Temperature dependence of self-diffusion coefficients of ions and solvents in ethylene carbonate, propylene carbonate, and diethyl carbonate single solutions and ethylene carbonate plus diethyl carbonate binary solutions of lipf6 studied by nmr. Journal of Chemical and Engineering Data, $57(7): 2012-2017,2012$.

[16] K. Hayamizu, Y. Aihara, S. Arai, and C. G. Martinez. Pulse-gradient spinecho h-1, li-7, and f-19 nmr diffusion and ionic conductivity measurements of 14 organic electrolytes containing lin(so2cf3)(2). Journal of Physical Chemistry B, 103(3):519-524, 1999.

[17] T. Hiemstra. Variable charge and electrical double layer of mineral-water interfaces: Silver halides versus metal (hydr)oxides. Langmuir, 28(44):1561415623, 2012.

[18] R. J. Hunter. Zeta Potential in Colloid Science, Principles and Applications. Academic Press, New York, 1981.

[19] H. S. Jang, D.-W. Park, and S. E. Shim. Measurement of dispersion stability of surface-modified nanosized carbon black in various liquids. J. Nanosci. Nanotechnol., 7(11):3827-3829, 2007.

[20] M. L. Jimenez, A. V. Delgado, and J. Lyklema. Hydrolysis versus ion correlation models in electrokinetic charge inversion: Establishing application 
ranges. Langmuir, 28(17):6786-6793, 2012.

[21] A. Y. Kim and J. C. Berg. Fractal aggregation: Scaling of fractal dimension with stability ratio. Langmuir, 16(5):2101-2104, 2000.

[22] K. Kondo, M. Sano, A. Hiwara, T. Omi, M. Fujita, A. Kuwae, M. Iida, K. Mogi, and H. Yokoyama. Conductivity and solvation of li+ ions of lipf6 in propylene carbonate solutions. Journal of Physical Chemistry B, 104(20):5040-5044, 2000.

[23] R. J. Kortschot, J. Lyklema, A. P. Philipse, and B. H. Erne. Diverging electrophoretic and dynamic mobility of model silica colloids at low ionic strength in ethanol. Journal of Colloid and Interface Science, 422:65-70, 2014.

[24] M. Kosmulski. Zeta potentials in nonaqueous media: how to measure and control them. Colloids and Surfaces A: Physicochemical and Engineering Aspects, 159(2-3):277-281, 1999.

[25] M. Kosmulski and E. Matijevic. Microelectrophoresis of silica in mixedsolvents of low dielectric-constant. Langmuir, 7(10):2066-2071, 1991.

[26] Y. N. Lin and P. Alexandridis. Temperature-dependent adsorption of pluronic f127 block copolymers onto carbon black particles dispersed in aqueous media. Journal of Physical Chemistry B, 106(42):10834-10844, 2002.

[27] H. Liu, S. Wang, Y. Xiao, Q. Yang, and X. Li. Charging behavior of carbon black in a low-permittivity medium based on acid-base charging theory. Journal of Materials Chemistry C, 3(16):3980-3988, 2015.

[28] B. M. Lowe, C.-K. Skylaris, and N. G. Green. Acid-base dissociation mechanisms and energetics at the silica-water interface: An activationless process. Journal of colloid and interface science, 451:231-44, 2015.

[29] J. Lyklema. Principles of interactions in non-aqueous electrolyte solutions. Current Opinion in Colloid \& Interface Science, 18(2):116-128, 2013.

[30] P.-C. Ma, N. A. Siddiqui, E. Maeder, and J.-K. Kim. Correlation between electrokinetic potential, dispersibility, surface chemistry and energy of carbon nanotubes. Composites Science and Technology, 71(14):1644-1651, 2011.

[31] L. Madec, M. Youssry, M. Cerbelaud, P. Soudan, D. Guyomard, and B. Lestriez. Electronic vs ionic limitations to electrochemical performance in 
li4ti5o12-based organic suspensions for lithium-redox flow batteries. Journal of the Electrochemical Society, 161(5):A693-A699, 2014.

[32] A. Martin-Molina, C. Calero, J. Faraudo, M. Quesada-Perez, A. Travesset, and R. H. Alvarez. The hydrophobic effect as a driving force for charge inversion in colloids. Soft Matter, 5(7):1350-1353, 2009.

[33] A. Ponrouch, E. Marchante, M. Courty, J.-M. Tarascon, and M. Rosa Palacin. In search of an optimized electrolyte for na-ion batteries. Energy \& Environmental Science, 5(9):8572-8583, 2012.

[34] A. Ponrouch, D. Monti, A. Boschin, B. Steen, P. Johansson, and M. R. Palacin. Non-aqueous electrolytes for sodium-ion batteries. Journal of $M a$ terials Chemistry A, 3(1):22-42, 2015.

[35] W. B. Russel, D. A. Saville, and W. R. Schowalter. Colloidal dispersions. Cambridge university press, 1991.

[36] B. Scrosati and J. Garche. Lithium batteries: Status, prospects and future. Journal of Power Sources, 195(9):2419-2430, 2010.

[37] A. Selmani, J. Lutzenkirchen, N. Kallay, and T. Preocanin. Surface and zeta-potentials of silver halide single crystals: ph-dependence in comparison to particle systems. Journal of Physics-Condensed Matter, 26(24), 2014.

[38] I. Semenov, S. Raafatnia, M. Sega, V. Lobaskin, C. Holm, and F. Kremer. Electrophoretic mobility and charge inversion of a colloidal particle studied by single-colloid electrophoresis and molecular dynamics simulations. Physical Review E, 87(2), 2013.

[39] K. E. Tettey and D. Lee. Effect of thermal treatment and moisture content on the charge of silica particles in non-polar media. Soft Matter, 9(30):7242$7250,2013$.

[40] V. A. Tolpekin, M. H. G. Duits, D. van den Ende, and J. Mellema. Stability ratio in binary hard sphere suspensions, measured via time-resolved microscopy. Langmuir, 19(10):4127-4137, 2003.

[41] P. C. Tsai, S. C. Chung, S. K. Lin, and A. Yamada. Ab initio study of sodium intercalation into disordered carbon. Journal of Materials Chemistry A, 3(18):9763-9768, 2015.

[42] J. W. Virden and J. C. Berg. The use of photon-correlation spectroscopy for estimating the rate-constant for doublet formation in an aggregating 
colloidal dispersion. Journal of Colloid and Interface Science, 149(2):528$535,1992$.

[43] Z. H. Wang, A. P. Ratvik, T. Grande, and S. M. Selbach. Diffusion of alkali metals in the first stage graphite intercalation compounds by vdw-dft calculations. RSC Advances, 5(21):15985-15992, 2015.

[44] K. Xu. Electrolytes and interphases in li-ion batteries and beyond. Chemical Reviews, 114(23):11503-11618, 2014.

[45] R. Xu, C. Wu, and H. Xu. Particle size and zeta potential of carbon black in liquid media. Carbon, 45(14):2806-2809, 2007.

[46] W. Xu, J. Xiao, J. Zhang, D. Wang, and J.-G. Zhang. Optimization of nonaqueous electrolytes for primary lithium/air batteries operated in ambient environment. Journal of the Electrochemical Society, 156(10):A773-A779, 2009.

[47] M. Youssry, L. Madec, P. Soudan, M. Cerbelaud, D. Guyomard, and B. Lestriez. Non-aqueous carbon black suspensions for lithium-based redox flow batteries: rheology and simultaneous rheo-electrical behavior. Physical Chemistry Chemical Physics, 15(34):14476-14486, 2013.

[48] M. Youssry, L. Madec, P. Soudan, M. Cerbelaud, D. Guyomard, and B. Lestriez. Formulation of flowable anolyte for redox flow batteries: Rheoelectrical study. Journal of Power Sources, 274(0):424-431, 2015.

[49] M. Zhu, J. Park, A. M. Sastry, and W. Lu. Numerical study of interaction and aggregation of non-spherical particles in forming li-ion battery cathodes. Journal of The Electrochemical Society, 161(9):A1247-A1252, 2014. 



\section{Chapter 3}

\section{Mechanical history}

\section{dependence}

Aditya Narayanan, Frieder Mugele, Michel Duits

The research described in this Chapter has been published as:

Mechanical History Dependence in Carbon Black Suspensions for Flow

Batteries: A Rheo-Impedance Study, Langmuir, 33(7):1629-1638, 2017

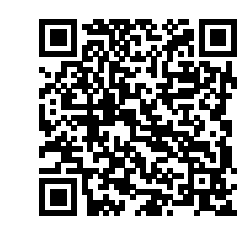




\subsection{Abstract}

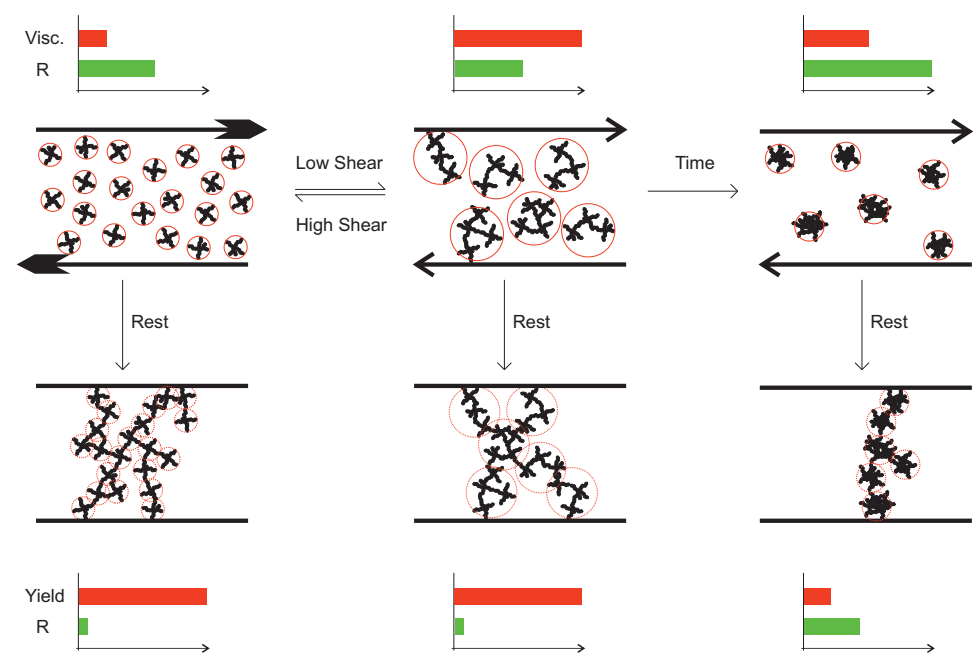

We studied the effects of shear and its history on suspensions of Carbon Black (CB) in lithium ion battery electrolyte, via simultaneous rheometry and electrical impedance spectroscopy. Ketjen Black (KB) suspensions showed shear thinning, rheopexy and exhibited a yield stress. Shear step experiments revealed a two timescale response. The immediate effect of decreasing the shear rate is an increase in both viscosity and electronic conductivity. In a much slower secondary response, both quantities change in the opposite direction, leading to a reversal of the initial change in the conductivity. Stepwise increases in shear rate lead to similar responses, in the opposite direction. This remarkable behavior is consistent with a picture in which agglomerating KB particles can stick directly on contact, forming open structures, and then slowly interpenetrate and densify. The fact that spherical CB particles show the opposite slow response, suggests that the fractal structure of the KB primary units plays an important role. A the- 


\subsection{INTRODUCTION}

oretical scheme was used to analyze the shear and time dependent viscosity and conductivity. Describing the agglomerates as effective hard spheres with a fractal architecture, and using an effective medium approximation for the conductivity, we found the changes in derived suspension structure to be in agreement with our qualitative mechanistic picture. This behavior of $\mathrm{KB}$ in flow has consequences for the properties of the gel network that is formed immediately after cessation of shear: both the yield stress and the electronic conductivity increase with the previously applied shear rate. Our findings thus have clear implications for the operation and filling strategies of semi solid flow batteries.

\subsection{Introduction}

In light of climate change, the recent years have seen a rapid adoption of renewable energy production ${ }^{4}$. Due to their inherently variable nature, renewables have placed considerable strain on the power grid which must match energy production to demand. A possible solution to this problem is to store energy in batteries ${ }^{40}$. Semi-Solid Flow Batteries (SSFBs), a recently developed configuration $^{9,11,13,14,21,22,34,44}$, are considered especially promising for such applications. SSFBs use two fluid electrodes, an anolyte and a catholyte in place of traditional solid electrodes. The use of fluid electrodes decouples the energy of a SSFB which depends on the size of storage tanks, from its power which depends on the size of the reactor. Additionally SSFBs may allow easy lifecycle management through the modification or replacement of their fluid electrodes.

SSFB electrodes are mixtures of conductive nanoparticles (CNPs) and electrochemically active particles (EAPs) dispersed in an electrolyte solution. In most 
SSFBs, the EAPs intercalate and de-intercalate lithium while the CNPs 'wire' the EAPs to the current collectors. The continuous phase, a mixture of linear and cyclic carbonate solvents with a high concentration of dissolved lithium salt, provides an ion conducting medium and a source of lithium ions. The CNPs used in SSFBs are typically superconductive carbon blacks such as Ketjen Black (KB) or Timcal SuperP ${ }^{9,47}$. These carbon blacks are sub-micron sized permanently fused aggregates of hollow spherical sub-units ${ }^{7,39}$.

The self-assembly of the CNPs, which depends on their morphology, their colloidal interactions and shear conditions, is of crucial importance to both the electrical and the mechanical performance of SSFBs. For colloidal carbon black (CB) units in SSFB media ${ }^{9,22,47,48}$ the Van der Waals attractions should be dominant, since their electrostatic interactions are strongly screened ${ }^{47,49}$. As a result, CB particles tend to form large cohesive structures. At rest (assuming a high enough concentration) they form a space filling network that can conduct electrons, and suspend EAPs against gravity through yield stress ${ }^{9,47}$. In flow (e.g. pumping, stirring) this network will be broken down into agglomerates. This leads to shear thinning and much lower electronic conductivity ${ }^{9,47}$. Henceforth in this manuscript we differentiate a permanently fused primary aggregate, from a reversibly flocculated cluster of these particles, which we will call an agglomerate.

Given the novelty of SSFBs, significant developments are still required to optimize their performance. Mechanical protocols should be a part of this optimization: fluids containing adhesive particles generally produce non-equilibrium structures, that can depend on mechanical history ${ }^{25,26,35,43}$. In this paper we focus on how shear and its history, influence the electrical and rheological properties of $\mathrm{CB}$ suspensions in a SSFB solvent. 


\subsection{INTRODUCTION}

While the case of CB in SSFB solvent is rather new ${ }^{9,47,49}$, we anticipate that some existing insights into the behavior of $\mathrm{CB}$ suspensions will also be applicable to our system. Suspensions of reversibly agglomerating colloids, including those of CB, are known to be strongly shear and history sensitive $e^{1,24,27,29,33,41,47}$. The storage modulus and yield stress of CB gels in oils have even been found to depend predictably on the pre-shear used to prepare them ${ }^{27,29}$. In flow, the viscosity of agglomerating colloidal suspensions typically decreases in time after an increase in shear stress or rate; a well-studied property known as thixotropy ${ }^{1,24}$. The opposite effect, a temporal increase in viscosity after an increase in shear stress or rate, known as rheopexy or anti-thixotropy, is much less common but has been observed in $\mathrm{CB}$ suspensions ${ }^{18,23,29,30}$. On a mechanistic level, rheopexy is thought to be caused by flow induced flocculation ${ }^{18}$ or by the ability of fractal structures to rearrange (when shear is lowered) into more densified agglomer$\operatorname{ates}^{27,29}$.

The electrical impedance of CB suspensions in shear flow has been studied much less often, but also here a dependence on the shear rate was found ${ }^{16,47,48}$. The study of the suspension's rheology and impedance in conjunction capitalizes on the tight connection of both behaviors to the (dynamic) microstructure of the agglomerates, which can be difficult to measure with optical or scattering techniques, especially for concentrated suspensions.

The present study makes use of a home-built rheo-impedance setup to characterize the influence of mechanical history on both rheological and electrical properties. This approach addresses both the practical aspect of optimizing SSFB performance via mechanical protocols, and the more fundamental aspect of understanding the underlying processes. Both stepwise changes in shear rate, and 
prolonged shear are explored to identify transient responses. Cessation of shear is included as a special case, where the system is left with only Brownian forces to possibly reorganize its structure. Our analysis of the structural changes at the microscale is supported by a theoretical model, in which the viscosity of effective hard-sphere agglomerates ${ }^{45}$ is combined with an effective medium theory for the conductivity $^{19}$.

\subsection{Materials and Methods}

\subsubsection{Suspension Preparation}

Ethylene carbonate (EC) and dimethyl carbonate (DMC) were obtained from Sigma Aldrich (anhydrous, 99\% + purity). Binary mixtures of EC and DMC were 1:1 by mass. LiPF6 was purchased from Alfa Aesar (98\% purity). Ketjen Black EC 600JD powder (KB) was obtained from AkzoNobel (The Netherlands). Thermax N990 powder was donated by Cancarb. Samples for SEM were dispersed in acetone, dried on silica wafers and then imaged. All other sample preparations and experiments were carried out in an MBraun Argon-filled glove box $\left(\mathrm{O}_{2}, \mathrm{H}_{2} \mathrm{O}\right.$ below $5 \mathrm{ppm})$.

The two types of CB used in this study differ in morphology (see also Fig. 3.8): for N990 the unit particles are more or less compact spheres, while for KB they are more fractal-like, as a result of fusion between spherical subunits. In this paper we designate the KB units as 'aggregates', while clusters formed via reversible flocculation are called 'agglomerates'. All suspensions of KB (of key interest for this work) were prepared at a concentration of $1 \%$ by weight $(\mathrm{wf}=0.01)$. As the KB aggregates are highly porous and their sub units are 
hollow shells, the occupied volume fraction in suspension is much higher than wf. A crude estimate can be obtained by multiplying wf into the ratio of the density of graphite $\left(2267 \mathrm{~kg} / \mathrm{m}^{3}\right)$ to the tap density of $\mathrm{KB}\left(100 \mathrm{~kg} / \mathrm{m}^{3}\right)$, giving a volume fraction of $23 \%$. Particles were first wetted by the 'pure' (binary) solvent in polypropylene containers. After 8 hours $\mathrm{LiPF}_{6}$ salt was added via a concentrated solution to bring its concentration to $1 \mathrm{M}$ (viscosity $4 \mathrm{mPas}^{8}$ ). After hand shaking, at least 8 hours was allowed to let the particles equilibrate. Samples were then homogenized by rotor stator mixing (Ultraturrax) at 15,000 rpm for 2 minutes, and subsequently loaded in the rheometer.

In exploratory experiments, we found similar trends as reported in this paper. However the quantitative behavior of KB suspensions in SSFB media appeared to be sensitive to preparation protocols. This could be related to the poor wetting by the solvents, sensitivity of the media ${ }^{49}$ and the strong dependence of macroscopic properties on the volume fraction (see S.I. Fig. 3.15 and $\operatorname{ref}^{47}$ ). Thus, for KB we performed all reported measurements (except those in Fig. 3.4) on the same sample.

\subsubsection{Rheoimpedance Measurements}

Parallel rheological and electrical measurements on CB suspensions were performed on a stress controlled Haake Rheostress RS600 rheometer with a homebuilt adaptation for Electrical impedance Spectroscopy (EIS) measurements (Fig. 3.1). A $60 \mathrm{~mm}$ diameter parallel plate geometry was designed with both stainless steel plates also acting as electrodes. The rheometer rotates and measures torques on the upper geometry. To allow a frictionless low-noise electrical connection to the rotating plate, a mercury based solvent trap was designed. A glass disk was 
used to electrically isolate the lower plate from the rheometer body. Due to its high thermal conductivity, glass also allows accurate temperature control (at 25 ${ }^{\circ} \mathrm{C}$ for all experiments). Argon was used to drive the rheometer air bearing.

EIS was performed using a four terminal configuration. The top plate was excited by a sinusoidal voltage and the bottom was connected to the virtual ground of a transimpedance pre-amplifier (HF2TA, Zurich instruments). A buffer preamplifier (HF2CA, Zurich instruments) was used to measure the potential difference between the plates. An impedance spectroscope (HF2IS, Zurich instruments) was used to extract the complex impedance from the current and voltage signals. AC frequency sweeps were performed from $10 \mathrm{MHz}$ to $100 \mathrm{MHz}$ (total time $550 \mathrm{~s}$ ) with a maximum applied voltage amplitude of $\sim 100 \mathrm{mV}$, which was within the linear response range of the samples. The frequency dependent impedance of the empty measurement setup was calibrated out using the 'open short' technique. Custom LabVIEW programs were used to synchronize rheological and electrical measurements.

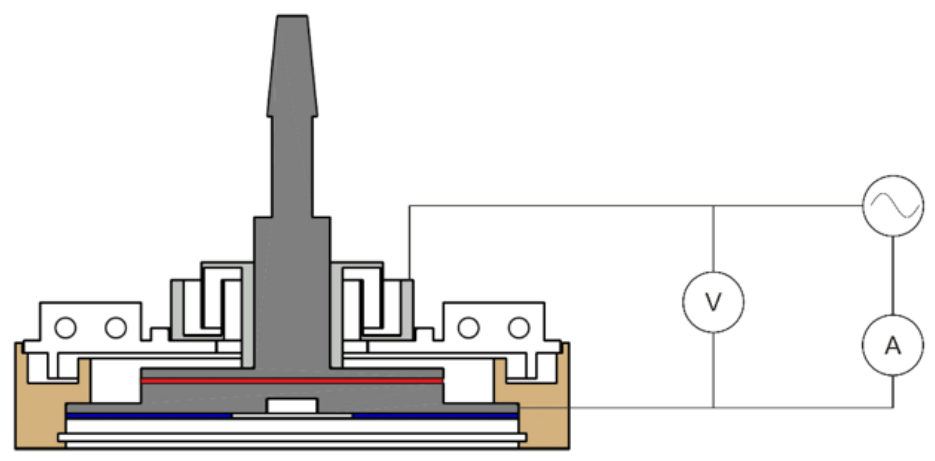

Figure 3.1: Schematic of rheo-impedance setup. Grey: Measuring geometry with stationary lower and rotating upper plate. Light Grey: Mercury solvent trap. Red: Sample. Blue: Isolating dielectric. Brown: Polypropylene body. White: Cover. 
Rheo-impedance experiments were performed with a plate-plate gap of $250 \mu \mathrm{m}$. Suspensions of CB have been reported to exhibit non-ideal rheometric behaviors such as wall slip, shear banding ${ }^{29}$ and vorticity alignment of flocs ${ }^{28}$, especially during start up and in low shear rate regimes. We avoided these effects in shear flow experiments by employing relatively high shear rates, where the samples behave as low viscosity liquids. To obtain a reproducible starting state (Fig. 3.2 ), the suspensions were pre-sheared at $10,000 \mathrm{~s}^{-1}$ for $200 \mathrm{~s}$, after which the shear rate was slowly ramped down to $316 \mathrm{~s}^{-1}$ and then held for $200 \mathrm{~s}$. Step changes in shear rate were followed by a dwell time $(\tau)$ which was different per type of experiment.

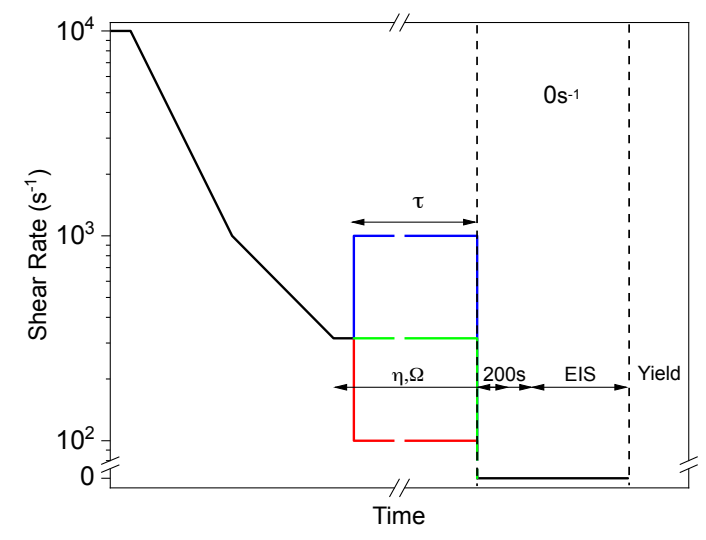

Figure 3.2: Schematic of the general experimental protocol for preshearing, and subsequent measurements of the electrical impedance and yield stress.

Measurements of the impedance during shear were done at a frequency of 0.5 $\mathrm{Hz}$ to allow real time monitoring of the electronic resistance (Sec. 3.3.3). Measurements after cessation of shear were done after a 200 s rest period. Impedance 
spectra were measured before the yield stress. In the measurement of the latter, the shear stress was ramped up at 62 s per stress decade while measuring the strain. The $\log ($ strain) versus $\log$ (stress) curve was fitted with two straight lines and the stress at the intersection was taken to be the yield stress ${ }^{36}$.

\subsubsection{Impedance Analysis}
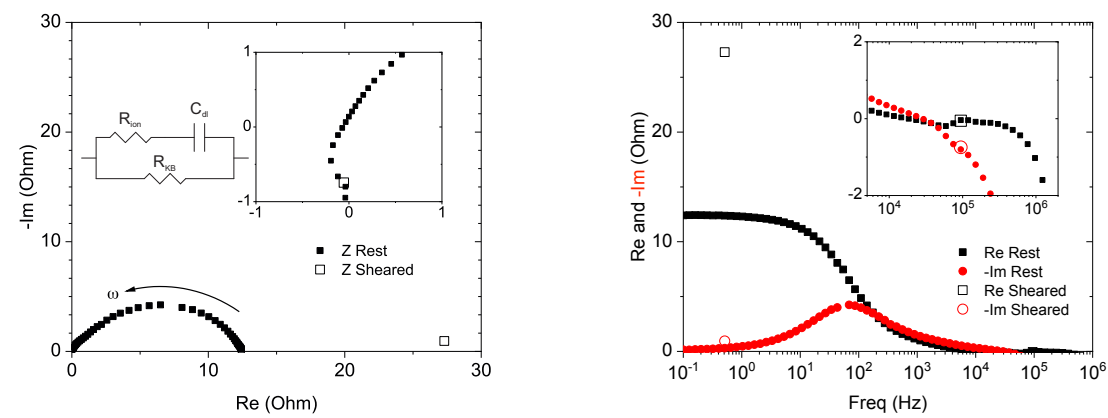

Figure 3.3: Nyquist with equivalent circuit (Left) and Bode (Right) plots of the impedance response of the KB suspension. Insets show a zoom around high frequency data $(<10 \mathrm{kHz})$. Filled symbols: KB at rest. Open symbols: KB under $1000 \mathrm{~s}^{-1}$ shear (only 2 frequencies measured). The $50 \mathrm{~Hz}$ points have been removed.

As flow battery electrodes contain conductive carbon in an electrolyte solution, they are capable of both ionic and electronic conduction. To monitor the KB network electrically, it is necessary to separate these contributions. This is done using $\mathrm{AC}$ impedance spectroscopy. The KB suspensions have two ionic charge carriers $\left(\mathrm{Li}^{+}, \mathrm{PF}_{6}{ }^{-}\right)$and an electron conducting carbon network. During measurements, the sample is in contact with electrodes that are ionically blocking but electronically reversible. Ignoring geometric capacitance, the response of this 
system can be simplified ${ }^{17}$ to that of an ionic resistor and an ionic double layer capacitance, both in parallel to the electronic resistance of the carbon black network. Thus the high frequency real impedance gives us the parallel combination of both resistances while the low frequency real impedance gives the electronic resistance. The data in Fig. 3.3 illustrate that the behavior of our KB suspension at rest can be analyzed with this approach. The very high frequencies (Fig. 3.3 insets) are affected by uncompensated parasitics ${ }^{2}$.

Under strong shear it is unlikely to have a continuous percolated network (for low shear rates, dynamic percolation may still be possible ${ }^{10}$ ). However, a measurement at two frequencies on the same KB suspension, but now under high shear (open symbols of Fig. 3.3), shows that the low frequency real impedance is finite while the imaginary impedance is close to zero. This implies that the sheared suspension has a finite electronic resistance ${ }^{17}$. Our measurements on other carbon black samples, show that the overall impedance response under shear is similar in shape to that at rest (see Fig. 3.16 in S.I.), justifying our use of a single low frequency to probe the electronic resistance. Additionally this is in qualitative agreement with earlier findings on Carbon Black filled polymers, which are known to conduct electrons via two mechanisms. At high volume fractions, when the network is continuous, conduction is graphitic ${ }^{37}$ (The resistance is still determined by constriction and ohmic contributions ${ }^{38}$ ). When the network is broken, the electrons can tunnel across small gaps $(<10 \mathrm{~nm})$ modulated by thermal voltage fluctuations ${ }^{37-39,47}$. For larger gaps however, the intrinsic electronic conductivity of the medium dominates ${ }^{38}$. 


\subsection{Results and Discussion}

\subsubsection{Intrinsic Flow curves}

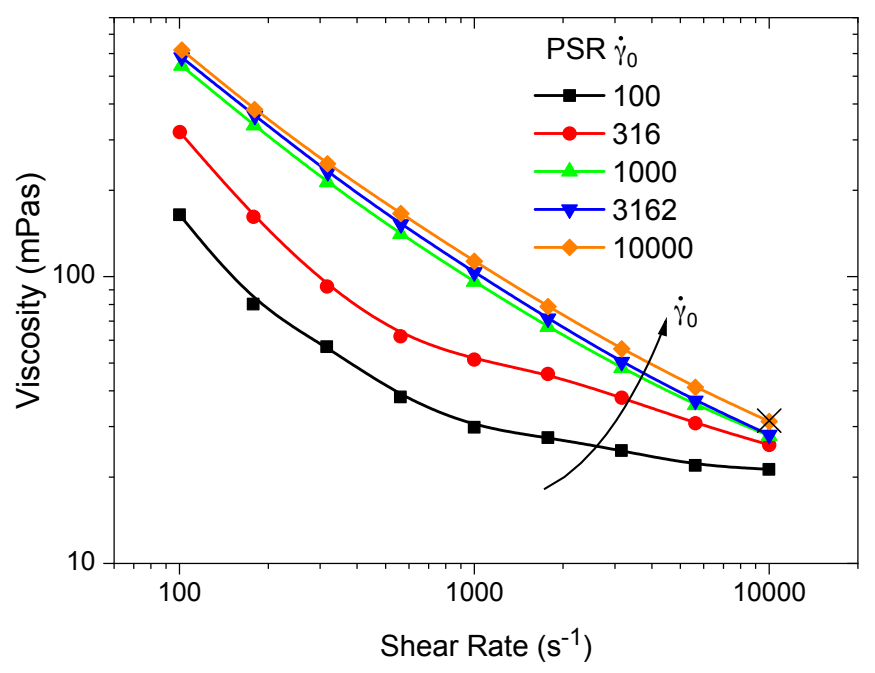

Figure 3.4: Intrinsic flow curve of agglomerates formed at different indicated shear rates (lines to guide the eye). The data point indicated by (x) represents the viscosity at $10000 \mathrm{~s}^{-1}$ after prolonged shearing.

We first consider the mechanical properties of our material in flow. To characterize KB, we measured a series of so called 'intrinsic' flow curves ${ }^{29}$, where each of the curves corresponds to a unique pre shear rate (PSR) that was maintained for $1000 \mathrm{~s}$ in order to reach a steady state. Data points within each curve were then obtained by stepping from the PSR to the shear rate of interest, and recording the viscosity quickly (as shown later, the viscosity almost immediately reached an extremum; it was this extremum that was recorded). All intrinsic 


\subsection{RESULTS AND DISCUSSION}

flow curves (Fig. 3.4) are strongly shear thinning, as expected for agglomerating suspensions $5,6,30,32,42,45$.

Their dependence on the PSR reveals interesting information that is specific to our KB system. Firstly, the three curves with the highest PSR coincide: indicating that a PSR of $1000 \mathrm{~s}^{-1}$ or more brings the material to a similar microstructural state. This suggests that the agglomerates are largely broken down under these conditions: generally for agglomerating systems a stronger shear flow causes more break-up. After prolonged (2000 s) shearing at the highest accessible shear rate of $10000 \mathrm{~s}^{-1}$ (from an arbitrary starting state) the sample showed a similar viscosity to the corresponding points of the intrinsic flow curves for PSR $\geq 1000 \mathrm{~s}^{-1}$ : this again suggests that the agglomerates are largely broken down (i.e. into primary aggregates) by prolonged shear above $1000 \mathrm{~s}^{-1}$. Secondly, it becomes clear that in order to reach a steady state, even the highest preshears need to be maintained for a significant duration, i.e. much longer than the data acquisition time (of $\mathrm{O}(1 \mathrm{~s})$ ) of the intrinsic flow curve. This is shown by the curves with PSR $\leq 316 \mathrm{~s}^{-1}$, for which the viscosities at are different from the curves at higher PSRs, even for measurement shear rates $\geq 1000 \mathrm{~s}^{-1}$. Thirdly, the fact that the intrinsic flow curves with smaller PSRs have lower viscosities is remarkable. In earlier suggested mechanistic pictures ${ }^{45}$, where shear leads to both breakdown and densification of agglomerates into smaller units, a lower PSRs should have resulted in higher viscosities, which is the opposite of what we observe. This points to a mechanism that can enhance the viscosity via agglomerate breakup in KB suspensions. The remarkable fact that the lowest possible viscosity (21 mPa.s) for our system was measured after the smallest preshear rate $\left(100 \mathrm{~s}^{-1}\right)$ also points in this direction. 


\subsubsection{Transient behaviors}

To examine this intriguing behavior, we performed experiments in which the material's response to shear rate steps was followed over time ${ }^{1}$. Both the viscosity and the electronic resistance (Sec. 3.3.3) were measured in parallel. All steps in shear rate were taken from the same reference condition, for we which we chose $316 \mathrm{~s}^{-1}$, based on Fig. 3.4. Some typical results of these measurements are found in Fig. 3.5, where both steps up (to $562 \mathrm{~s}^{-1}$ and $1000 \mathrm{~s}^{-1}$ ) and down (to 177 $\mathrm{s}^{-1}$ and $100 \mathrm{~s}^{-1}$ ) are illustrated.

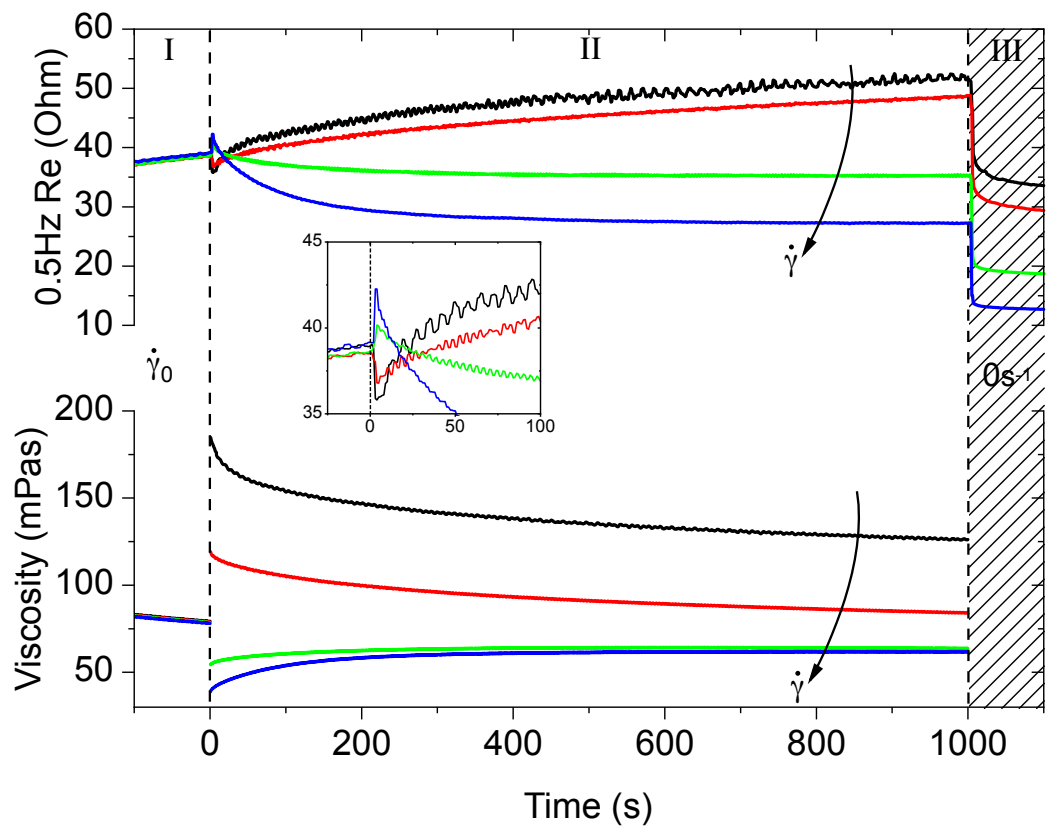

Figure 3.5: Evolution of viscosity and low frequency real impedance of KB suspension after a shear rate step from $316 \mathrm{~s}^{-1}$ to $100 \mathrm{~s}^{-1}$ (black), $177 \mathrm{~s}^{-1}$ (red), $562 \mathrm{~s}^{-1}$ (green), $1000 \mathrm{~s}^{-1}$ (blue). The inset is a zoom into the response of the electronic resistance around the step. 


\subsection{RESULTS AND DISCUSSION}

Both the viscosity and the electronic resistance show a two-staged response. The initial response, a large increase in viscosity and a small decrease in electronic resistance for a step down in rate (transition from region I to region II) is fast, with a timescale of $\mathrm{O}(1 \mathrm{~s})$. It is not resolvable, considering the short time that the rheometer needs to step the shear rate, and the low frequency of the impedance measurement. For the steps to higher shear rates, the opposite trends were observed. The secondary response (region II) is significantly slower O (100-1000 s). The slow changes in the viscosity and resistance appear to have similar time scales, suggesting that they both probe changes in the microstructure of the KB.

Remarkably, for both signals, the secondary response opposes the direction of the initial response. For the viscosity this amounts to a relatively small correction (rheopexy, shear thinning), but for the electronic resistance the secondary effect is strong and causes a reversal of the overall effect. The shape of the decays of the viscosity and electronic resistance is well described by stretched exponentials; such behavior has been found for the viscosity of thixotropic systems ${ }^{1}$. The 'opposing change' in the second stage is found irrespective of whether the shear rate is increased or decreased. Yet the direction in which the shear rate is changed, still determines the 'sign' of all changes. This is consistent with a picture in which all structural transitions can be reversed via the shear rate. 


\subsubsection{Mechanistic Picture}

We interpret our findings in Figs. 3.4 and 3.5 with a picture in which KB reversibly agglomerates via two distinct mechanisms (each with its own timescale and influence on the microstructure). Both the viscosity and electronic resistance depend on the concentration and morphology of the agglomerates, but in different ways.

Flow curves of weakly agglomerating suspensions have been successfully described by modeling the agglomerates along with their immobilized solvent as effective hard spheres ${ }^{12,32}$. Shear thinning is then explained via a lowering of the effective hard sphere volume fraction. As the shear rate is increased, higher shear stress causes the agglomerates to break down into smaller structures. Because of the 'fractal' build up, the latter then occupy less total volume (see Fig. 3.6). Although (strictly speaking) perfectly fractal scaling is rarely observed, it is has often been found that the structure of agglomerates can be fairly well described with this concept $12,32,42,45$.
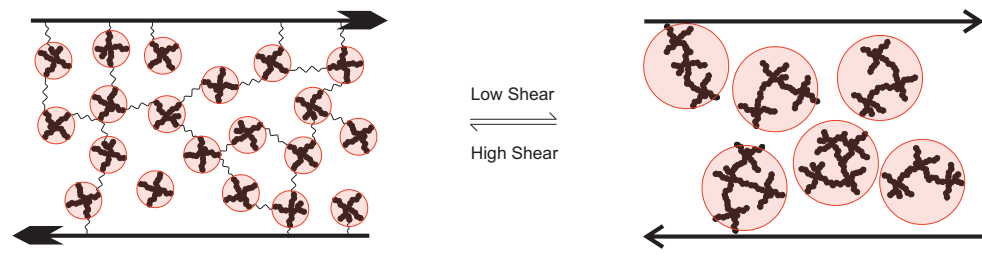

Figure 3.6: Cartoon of fractal agglomeration and imperfect percolation (left) in shear flow.

To understand the (changes in) electronic conductivity of the suspension under flow, we need to take into account the formation of (transient) pathways with missing links (Fig. 3.6, left). As such gaps dominate the resistance (Sec. 3.3.3), 
the macroscopic electronic resistance of the sheared suspension should be determined by the concentration of agglomerates and their typical distance of closest approach.

We now proceed to a qualitative interpretation of Fig. 3.5, focusing first on the instant process. The jumps in viscosity and electronic resistance may be explained by agglomeration due to lower shear forces when the rate is stepped down. The immediate sticking between colliding fractal entities leads to more open agglomerates, which enclose (and hence immobilize) more solvent, causing the hydrodynamic volume fraction and thus the suspension viscosity to increase (note: if the shear step were small then a binary collision would lead to an unstable agglomerate, which would be quickly eroded). The same agglomerate restructuring also changes the ensemble of interrupted conductive pathways. The formation of fewer but larger agglomerates after a step down in shear rate, diminishes both the average gap between agglomerates, and the number of gaps an electron needs to traverse along a conductive path. Both these effects should lower the electronic resistance. However they are counteracted by a decrease in the number of electronic pathways. This could explain why the overall decrease in resistance is small.

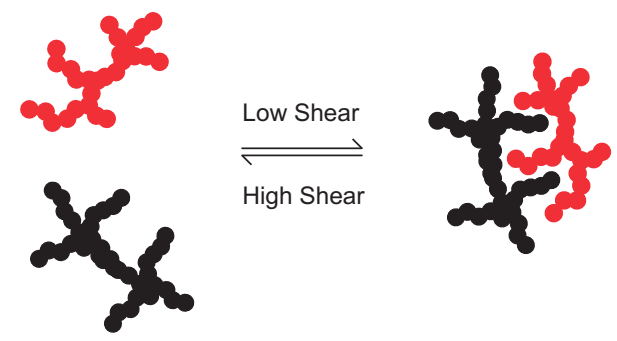

Figure 3.7: Cartoon of interpenetrating agglomeration. 
As shear is continued after the step down in rate, the population of agglomerates is rejuvenated continually: bonds between $\mathrm{KB}$ units are incessantly broken and formed by shear. This provides a means for the agglomerates to overcome kinetic barriers and discover more stable states. As the unit particles (and their agglomerates) are (roughly) fractal in nature, the randomness of collisions will occasionally cause two particles to interpenetrate (see Fig. 3.7). Due to the enhanced contact area and generally lower aspect ratio, these agglomerates will be more stable (compared to those formed by peripheral contacts). However if the shear stress gets high enough, they can be torn apart again, making the process reversible. Clearly, a consolidated interpenetration should lead to an overall densification of agglomerates, leading to a lower hydrodynamic volume fraction and hence a reduced viscosity. It also follows from mass conservation that the average gap between agglomerates will increase in this scenario. Additionally the number of conduction pathways will decrease. Both effects should lead to the observed increase the electronic resistance (after a stepdown in shear rate).

The dependence of the interpenetrating agglomeration on shear rate can be understood as follows: the stability of interpenetrating KB units will depend on how deeply they are lodged within the agglomerate, as only the peripheral zone will be exposed to local shear flow. This explains why complete reversal of the process can only be achieved at high shear rates: here not only the shear stresses are high, but also the agglomerates are small, thus exposing all KB units to the shear (note: this is also why we defined our samples by preshearing them at 1000 $\left.\mathrm{s}^{-1}\right)$.

In our proposed mechanism of interpenetrating agglomeration, the shape of the KB units plays an important role. To test this hypothesis, we performed an 


\subsection{RESULTS AND DISCUSSION}
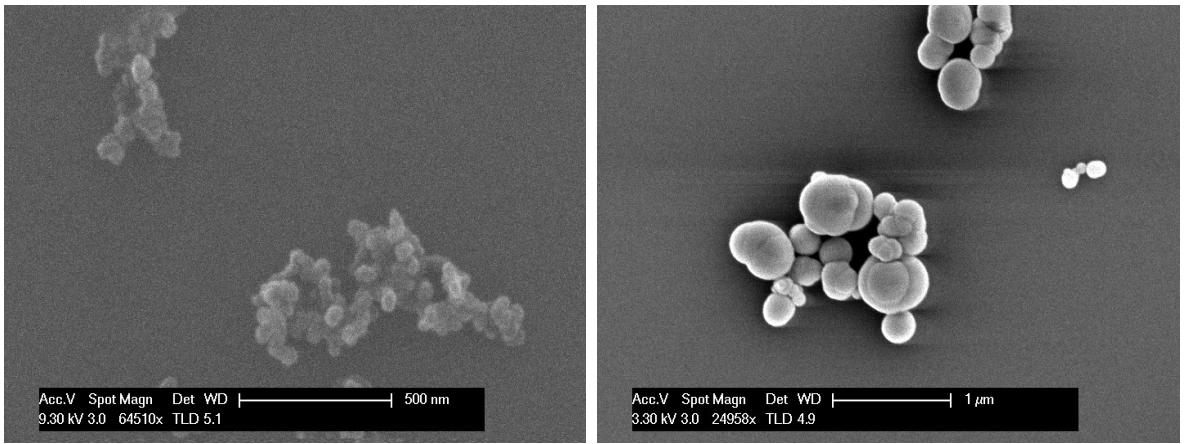

Figure 3.8: SEM images of Left: Ketjen Black Right: N990. Particles are agglomerated due to drying.

experiment comparable to that in Fig. 3.5 but now with spherical unit particles (N990). This system was also strongly shear thinning, indicating reversible agglomeration. A weight fraction of $30 \%$ was chosen to achieve a high shear viscosity similar to KB. Assuming that the hydrodynamic volume fractions of both suspensions are comparable when they are broken down to the unit particles, the only relevant difference should be the shape of the unit particle (see Fig. 3.8).

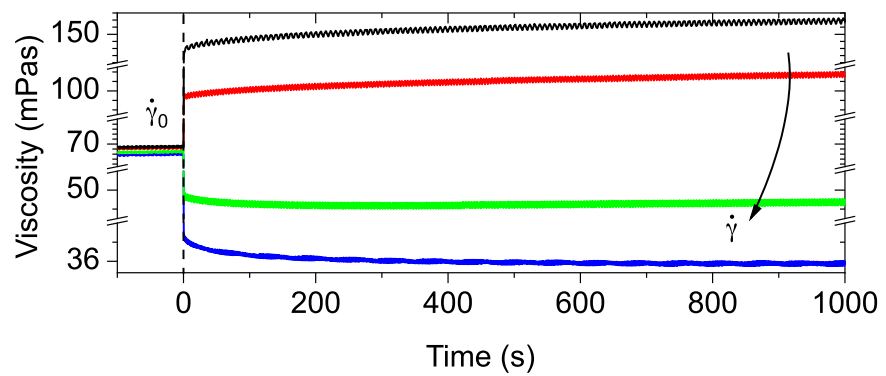

Figure 3.9: Evolution of viscosity of $N 990$ suspension after a shear rate step from $316 \mathrm{~s}^{-1}$. The colors correspond to shear rate steps described in Fig. 3.5. Viscosity axis breaks and their scales are not uniform. One tick corresponds to 1 mPas. 
The electrical impedance of the N990 suspensions turned out to be too high to be accessible at experimental frequencies $(<1 \mathrm{mHz})$. As shown in Fig. 3.9, similar to KB the N990 suspension also exhibits an immediate jump up in viscosity when the shear rate is stepped down, consistent with instant open agglomeration. However when shear is continued, the viscosity slightly increases (thixotropy, shear thinning), in contrast to KB where it decreases. This supports our explanation that the fractal morphology of KB is responsible for the occurrence of interpenetrating agglomeration.

\subsubsection{Modeling}

To further examine the mechanistic explanations proposed so far (as sketched in Figs. 3.6 and 3.7), we combined previously developed concepts for describing the viscosity of agglomerating systems ${ }^{45}$ and the conductivity of filled polymers ${ }^{38}$ into a simplistic theoretical scheme. A complete description of this scheme is given in the Appendix. The scheme uses the measured viscosity and electronic resistance as inputs, and outputs the collision radius and fractal dimension of the agglomerates (along with other derived geometrical properties). The KriegerDougherty equation ${ }^{20}$ is used to calculate the hydrodynamic volume fraction from the relative viscosity, while part of the Potanin model ${ }^{32,45}$ is used to set a relation between the agglomerate radius and the fractal dimension. An effective medium approach for the electronic resistance is used to construct an additional relation between the agglomerate radius and the fractal dimension. In this approach, agglomerates are assumed to occupy centers in a lattice. The resistivity of the suspension then depends on resistance between adjacent occupied lattice sites and asymptotically on the fraction of occupied sites. Since the agglomerates 


\subsection{RESULTS AND DISCUSSION}

have a fractal architecture, the resistance of a 'conductive contact' depends not only on proximity but also on the local (sub) unit particle density in the outer shells of the agglomerate.

The above relations are solved numerically for each measured viscosity and electronic resistance combination to yield a solution (fractal dimension, collision radius). Several other structure parameters can then be derived. Application of the scheme to the measurements of Fig. 3.5 leads to the data shown in Fig. 3.10. We observe that the trends used in our qualitative interpretation are reproduced by the model. For steps down in shear rate, the fast restructuring response leads to larger and less dense agglomerates while reducing both their concentration and spacing. During the slow interpenetrating agglomeration process, densification leads to a strong increase in the inter-agglomerate distance (and hence electronic resistance). For steps up in shear rate, the opposite trends occur, in line with expectations.
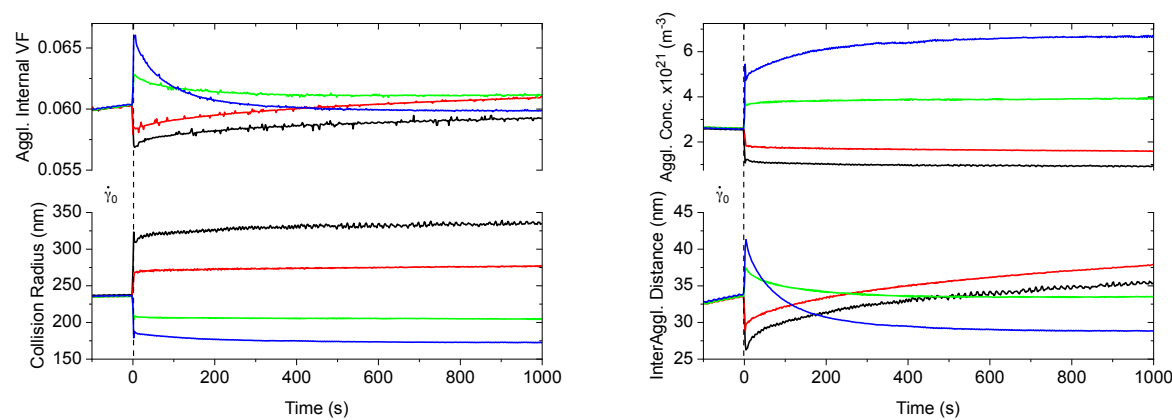

Figure 3.10: Analysis of the experiments of Fig. 3.5 with our theoretical model. Shear rate steps are made from $316 s^{-1}$ to $100 s^{-1}$ (black), $177 s^{-1}$ (red), 562 $s^{-1}$ (green), $1000 s^{-1}$ (blue). 


\subsubsection{Cessation of shear}

We revisit the experiment shown in Fig. 3.5, now focusing on the last stage (region III), in which the flow is terminated after $1000 \mathrm{~s}$ of maintained shear at a constant rate. The (still monitored) electronic resistance shows an immediate sharp drop, indicating the formation of a space-filling network. After $200 \mathrm{~s}$ the resistance reaches a steady state, justifying measurements of the full impedance spectra (see Fig. 3.17 in S.I.) and the yield stress. Remarkably, both the low frequency real impedance and the yield stress show a systematic dependence on the previously applied shear rate, with higher preshear leading to a higher yield stress and a lower electronic resistance (Fig. 3.11). This effect is of strong significance to SSFBs where a high yield stress and low electronic resistance are both required for efficient operation

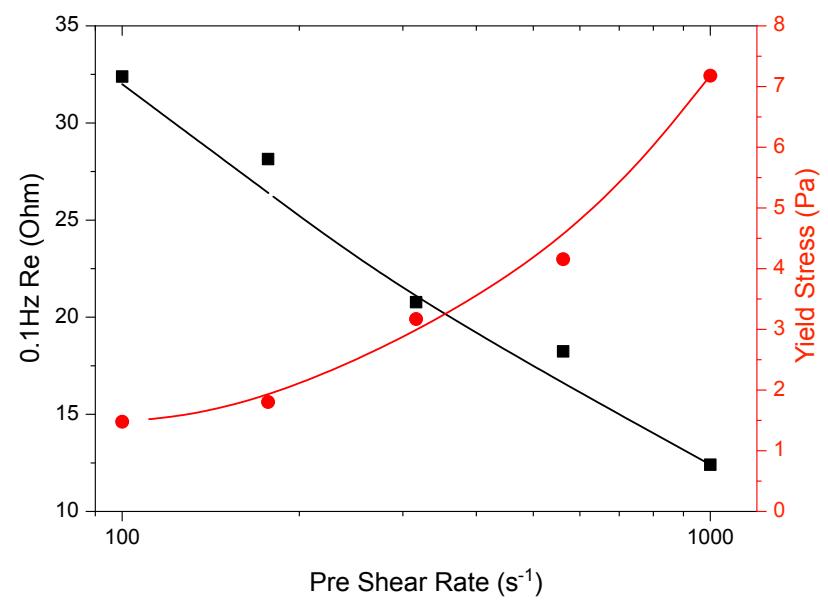

Figure 3.11: Rest impedance and yield stress of KB suspensions after preshearing for $1000 \mathrm{~s}$ at indicated rates (lines to guide the eye). 


\subsection{RESULTS AND DISCUSSION}

To explain these trends, we propose that the agglomerate structures as defined by the shear history, are the 'building blocks' of the solid network that forms after shear cessation. In other words, the agglomerates remain intact up to the point where they connect with other agglomerates, to form a space-filling network. This 'freezing in' effect is plausible, as agglomerates can still undergo Brownian motion ${ }^{3}$ while thermal forces are too weak to break bonds between the KB units.

The qualitative dependences of the electronic resistance and yield stress on the preshear rate can be rationalized using the same shear (history) induced changes in the agglomerate structure as discussed earlier. High preshears result in small agglomerates, which assemble into a highly branched network when shear is stopped. Conversely, pre-shearing at low rates leads to networks with fewer and thicker branches, because the agglomerates were larger in size and fewer in number. The contact areas between the agglomerates that form the low preshear gel should be similar (peripheral contacts) and thus no more effective at transferring charge and mechanical force than those of the high pre-shear gel. However in the latter material, the number density of branches will be higher, leading to a higher yield stress and a lower electronic resistance.

To further examine the role of the secondary response (region II in Fig. 3.5) in light of the results of Fig. 3.11, we explored the effect of shear cessation at different times (Fig. 3.12 left). As before, a 200 s rest time was given between the cessation of shear and the measurement of the impedance spectrum (complete spectrum in S.I. Fig. 3.18). The yield stress was subsequently measured. As shown in Fig. 3.12 right, both the final electronic resistance and yield stress were found to evolve in an $\mathrm{S}$ shape from their starting value (in the case of the step to $100 \mathrm{~s}^{-1}$ steady state is not completely reached even after $1000 \mathrm{~s}$ ). 

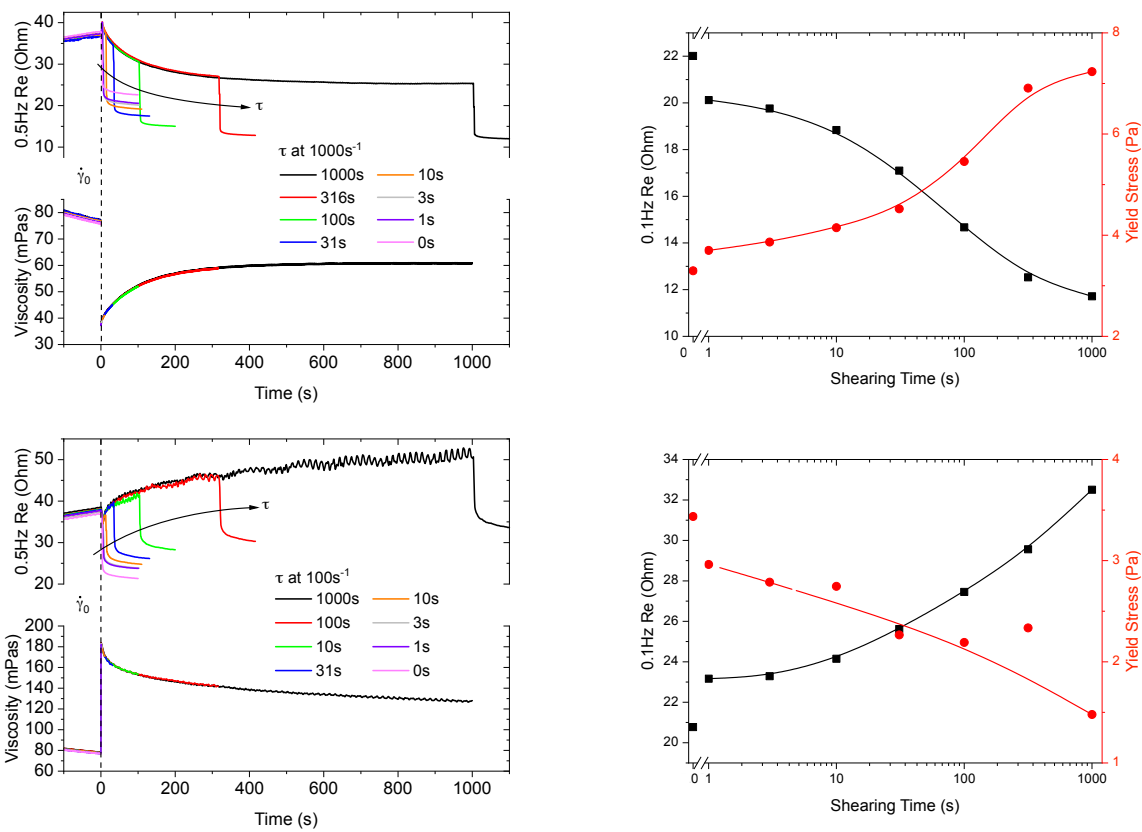

Figure 3.12: Left: Evolution of viscosity and low frequency real impedance after a shear rate step from $316 \mathrm{~s}^{-1}$ to $1000 \mathrm{~s}^{-1}$ (up) or $100 \mathrm{~s}^{-1}$ (down) with shear cessation at different (indicated) times. Right: Rest network resistance and Yield stress measured after shear cessation at indicated time (lines to guide the eye).

Remarkably, their values for just one second of shear after the step are fairly similar to those without the step. This indicates that the structures of the gelled networks must also be very similar, despite the instant agglomeration (viscosity and resistance jumps) that occurred during the one second step. This suggests that the instant agglomeration process and gel formation on shear cessation have similar mechanisms. Additionally it shows that the process responsible for the secondary response (interpenetration) is almost exclusively responsible for the change in rest network properties. 


\subsection{Conclusion}

The influence of shear and its history on the rheology and electrical impedance of Ketjen Black suspensions in SSFB solvent was studied with two objectives; to understand the microscopic origin of the shear history dependent behaviors, and to identify the implications for the performance of SSFBs. Both aspects revolve around the microstructure of the agglomerated suspension, and how it adapts to mechanical conditions. Given the difficulty of measuring the relevant structural properties directly via microscopy or scattering, our simultaneous study of the rheology and electrical impedance provided a good alternative method.

The fast and slow transient responses to a change in shear rate clearly indicate that KB agglomerates exhibit two build up (breakup) mechanisms after decreasing (increasing) the shear rate. The fast response was attributed to open agglomeration while the slower mechanism was ascribed to a interpenetration of the fractal-like KB units. The combination of these processes leads to a shearand time-dependent agglomerate structure, which gets 'frozen in' on cessation of flow, with a higher preshear leading to a stronger gel with a lower electronic resistance, both critical to SSFB performance.

An implication of our findings is, that for SSFBs operated in absence of flow, a high pre-shear (through fast pumping or stirring) would be the best for minimizing the electronic resistance, and maximizing the yield stress. For SSFBs operated in continuous flow, a high flow rate would minimize the electronic resistance. We expect that our findings will be of relevance not just to SSFBs but also to other systems which use fractal carbon blacks such as flow supercapacitors. Additionally, we foresee electrochemical cycling to play an important role on the rheo-impedance of fluid electrodes (and vice-versa) and are currently 
investigating the same.

\subsection{Appendix}

In this section we describe a scheme for calculating the structure in an agglomerated suspension, using measured data for the viscosity and conductivity as input.

To describe the viscosity, we model the fractal agglomerates as effective hard spheres with a hydrodynamic radius $R_{h}$ which depends on the shear rate and history. The Krieger-Dougherty equation ${ }^{20}$ is used to calculate the hydrodynamic volume fraction $\phi_{h y d}$ from the viscosity $\eta$ :

$$
\eta=\eta_{0}\left(\frac{\phi_{h y d}}{\phi_{m h y d}}\right)^{-2.5 \phi_{h y d}}
$$

where $\eta_{0}$ is the solvent viscosity and $\phi_{m h y d}$ is the maximum hydrodynamic volume fraction. From scaling laws for fractal agglomerates made of spherical sub-units (of radius $a$ ), the number $N$ of subunits enclosed by a shell of radius $R$ is given by $^{45}$ :

$$
N=N_{0}\left(\frac{R_{c}}{a}\right)^{d_{f}}
$$

Note that we do not distinguish between subunits originating from different primary aggregates. Setting $N_{0}$ to unity for simplicity and invoking mass conservation, $R_{h}$ can be related to $d_{f} 45$.

$$
\begin{gathered}
R_{h}=\kappa a\left(\kappa^{-d_{f}} \frac{\phi_{\text {hyd }}}{\phi_{\text {particles }}}\right)^{\frac{1}{\left(3-d_{f}\right)}} \\
\kappa=\frac{R_{h}}{R_{c}}
\end{gathered}
$$


Where $\phi_{\text {particles }}$ is the physical volume fraction of the particles; in our case the volume fraction of the solvent filled $\mathrm{KB}$ sub units. $R_{c}$ the radius of the smallest sphere that completely encloses the agglomerate; the collision radius. $\kappa$ is the ratio of the hydrodynamic to collision radius and is $\beta / \sqrt{1+2 / d_{f}} 45$. This equation contains two unknowns $\left(R_{c}, d_{f}\right)$ and thus we use the impedance data to impose an additional constraint.

The electronic resistance of the CB suspension is governed by the macroscopic paths which electrons can take (percolation) and the conductivities of the paths themselves, i.e. the suspension behaves as a network of resistors. The effective medium approximation can be used to obtain an analytical expression for the conductivity of the entire system ${ }^{19}$. It consists of replacing the random conductances by a single value $G_{m}$ such that the overall electrical properties are unchanged. Sheared concentrated CB suspensions present a rather special case, as the concentration is high enough that conductive chains can form, however the shear keeps these chains from being continuous; despite 'percolation', the electrons still need to cross insulating gaps between agglomerates. We assume that CB agglomerates behave as conductive spheres whose centers are randomly distributed in a lattice. As the electronic conductivity of carbon is much higher than that of the solvent, we assume a binomial probability distribution of conductivities between lattice sites; If both neighboring sites are occupied the conductance $G_{l}$ will be finite; otherwise it will be zero. From the effective medium approximation it then follows that:

$$
G_{m}=\frac{\frac{z}{2} \frac{\phi}{\phi_{m}}-1}{\frac{z}{2}-1} G_{l}
$$


where $z$ is the coordination number of the lattice, $\phi$ is the lattice occupation fraction and $\phi_{m}$ its maximum value (taken equal to that of their hydrodynamic counterparts for simplicity). For a lattice edge length of $l$, the suspension resistivity $\rho$ then becomes:

$$
\rho=\frac{l}{G_{l}} \frac{\frac{z}{2}-1}{\frac{z}{2} \frac{\phi}{\phi_{m}}-1}
$$

In our case $l$ is equal to the total of the agglomerate diameter $2 R_{c}$ and the (typical) gap between agglomerates $d$. The latter is given by ${ }^{15}$ :

$$
d=2 R_{c}\left(\left(\frac{\phi_{m}}{\phi}\right)^{1 / 3}-1\right)
$$

The conductivity $\sigma_{l}$ of the 'gapped connection' depends on the geometry and the prevailing conduction mechanism. Agglomerates separated by very small gaps (a few $\mathrm{nm}$ ) conduct through fluctuation induced quantum tunneling ${ }^{39}$. For larger gaps the (low) intrinsic electronic conductivity of the solvent dominates. As inter-agglomerate distances of tens of nanometers are expected (shown later), $G_{l}$ is given by:

$$
G_{l}=\sigma_{s} \frac{a_{c t}}{d}
$$

Where $\sigma_{s}$ is the intrinsic electronic conductivity of the solvent and $a_{c t}$ is the charge transfer area. To determine $a_{c t}$ we assume that conduction is effective upto a depth $h$ into the agglomerate as shown in figure 3.13.

From the geometry of the system it is evident that:

$$
a_{c t}=F \pi 2 h R_{c} \quad h<<R_{c}
$$




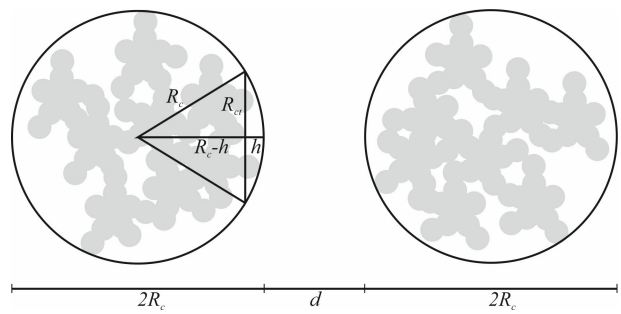

Figure 3.13: Geometry of the lattice cells.

Where $F$ accounts for the fact that the perimeter of the agglomerate is not completely occupied. To evaluate $F$ we consider a shell of thickness $d R_{c}$ around the fractal of radius $R_{c}$. The number of primary particles enclosed in this shell $d N$ is given by:

$$
d N=d_{f} \frac{R_{c}^{d_{f}-1}}{a^{d_{f}}} d R_{c}
$$

To get the area fraction $F$ we simply divide the projected area of these particles by the surface area of the sphere of radius $R_{c}$.

$$
a_{c t}=\frac{\pi}{2} \frac{d_{f} R_{c}^{d_{f}-2} h d R_{c}}{a^{d_{f}-2}}
$$

Collecting the above relations, we get an equation for the impedance of the suspension:

$$
\rho=k \frac{a^{d_{f}}\left(2 R_{c}+d\right) d}{d_{f} R_{C}^{d_{f}-2}} \frac{1-\frac{2}{z}}{\frac{Z}{2} \frac{\phi}{\phi_{m}}-1} \quad k=\frac{2}{a^{2} h d R_{c} \pi \sigma_{S}}
$$

Equation 3.3 and 3.11 can be numerically solved to obtain $d_{f}$ and $R_{c}$. To make a numercial analysis of the data in Fig. 3.5, some numerical constants need to be specified; these can be found in Table 3.1. To obtain the magnitude of $\phi_{\text {particles }}$ 
in Eq. 3.3, we use the assumption that a sufficiently prolonged shearing at 1000 $\mathrm{s}^{-1}$ the agglomerates are completely broken down.

\begin{tabular}{lll} 
Parameter & Value & Source \\
\hline$\eta_{0}$ & $4 \mathrm{mPas}$ & Ref. $^{8}$ \\
$a$ & $5 \mathrm{~nm}$ & Ref. $^{39}$ \\
$\phi_{\text {mhyd }}$ & 0.71 & High shear limit for \\
& & hard spheres \\
$\beta$ & 0.72 & Ref. $^{46}$ \\
$z$ & 12 & \\
$d_{f}$ primary aggregate & 1.8 & Ref. $^{7}$ \\
$R_{h}$ primary aggregate & $90 \mathrm{~nm}$ & DLS (See S.I. Fig. 3.14)
\end{tabular}

Table 3.1: Parameter estimates used in model. 


\subsection{Supplementary Information}

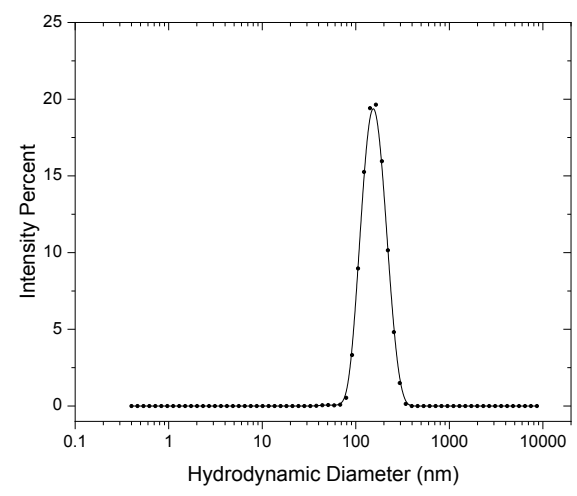

Figure 3.14: Characterization of a dilute $K B$ suspension in $E C: D M C$ (1:1, no salt) using by Dynamic Light Scattering with a Malvern Zetasizer. Prior to the measurement, the sample was subjected to ultrasonication, to break up the agglomerates.
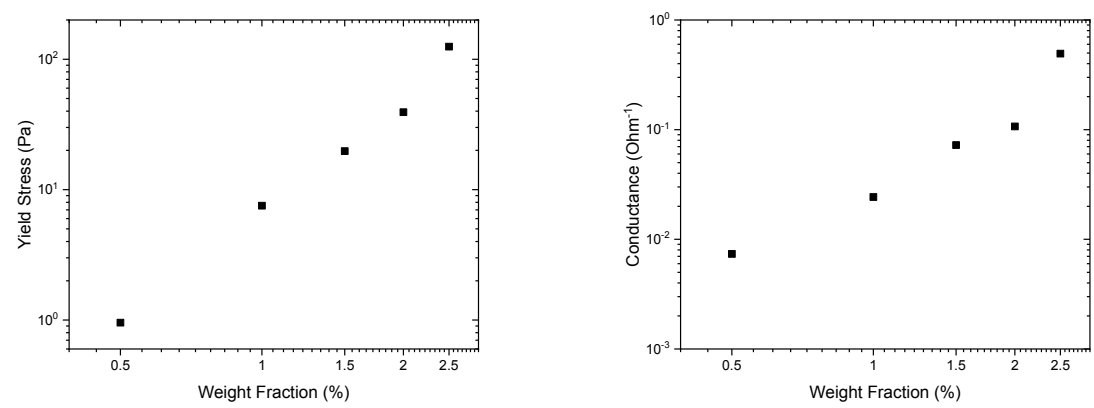

Figure 3.15: Concentration dependence of the yield stress and real part of the electrical impedance at $0.1 \mathrm{~Hz}$, for several KB slurries. Prior to the measurements, a preshear rate of $1000 \mathrm{~s}^{-1}$ was applied for $200 \mathrm{~s}$. 

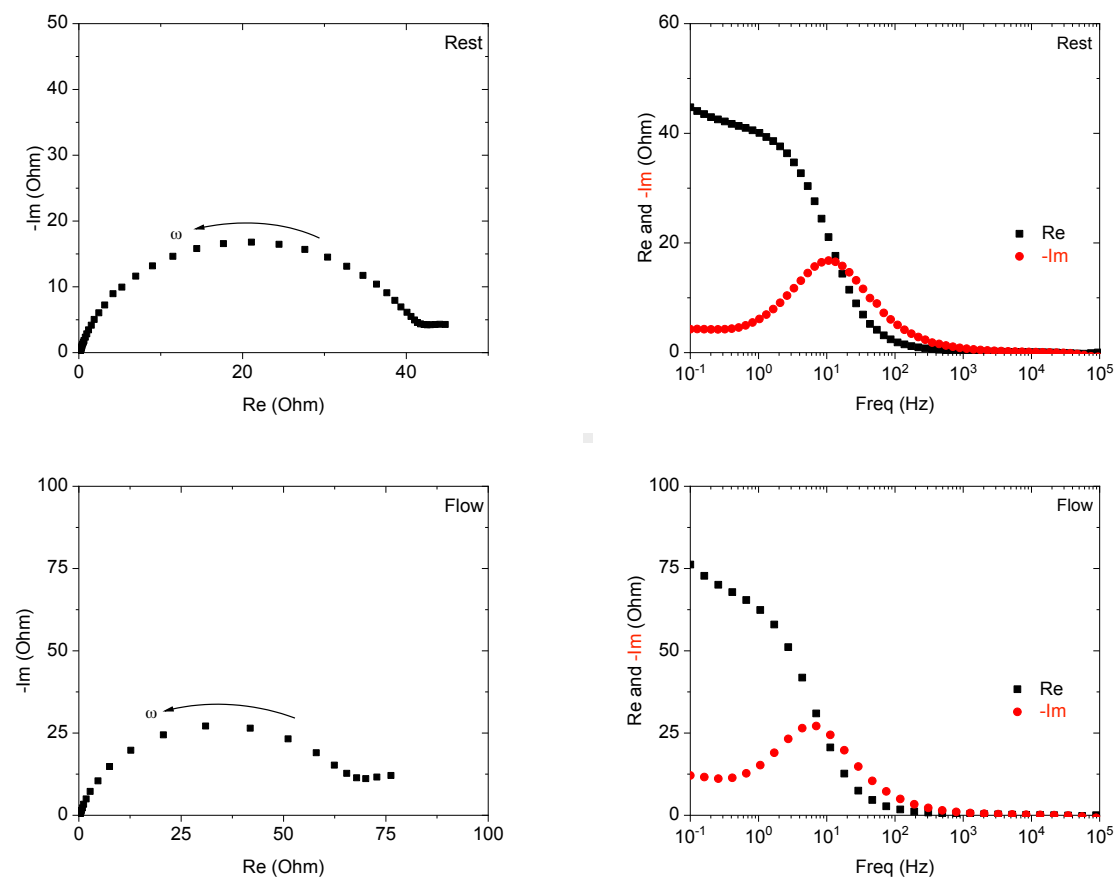

Figure 3.16: Top; Nyquist (Right) and Bode (Left) plots of the impedance response of different $K B$ suspensions (of the same make-up) at rest. Bottom; Nyquist(Right) and Bode(Left) plots of the response at a shear rate of $1000 \mathrm{~s}^{-1}$. At the lowest frequencies the impedance shows a slight deviation (incomplete secondary arc). This may be a consequence of a higher interfacial electron transfer impedance ${ }^{17}$ in this sample. 


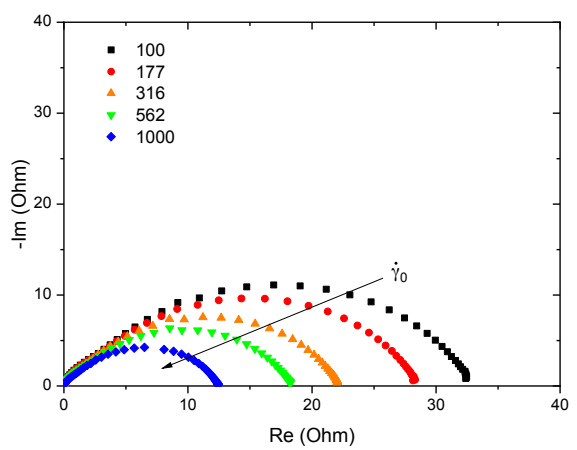

Figure 3.17: Impedance spectra of 1 wt \% KB suspensions, measured after cessation of shear. Samples were presheared for $1000 \mathrm{~s}$ at the indicated rates (lines to guide the eye).
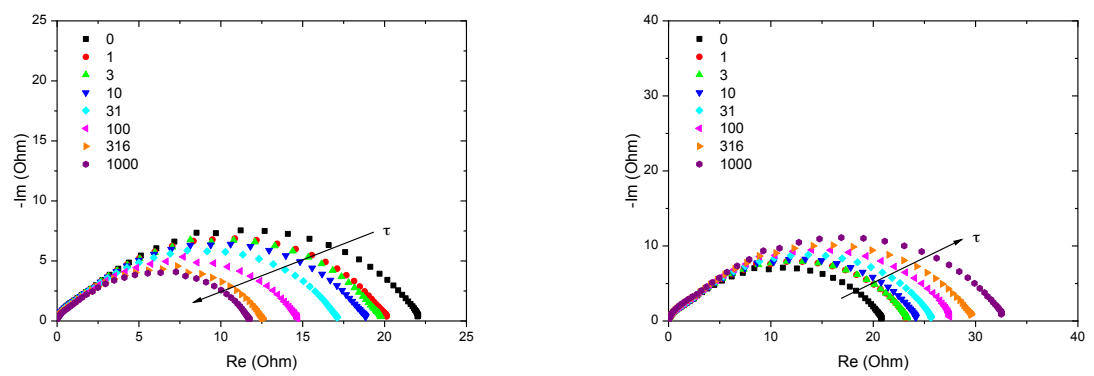

Figure 3.18: Impedance spectra of 1 wt \% KB suspensions, measured after cessation of shear. Mechanical history: samples were initially sheared at $316 \mathrm{~s}^{-1}$, after which the shear rate was either increased to $1000 \mathrm{~s}^{-1}$ (left) or $100 \mathrm{~s}^{-1}$ (right). The duration of the second shear period was varied; these durations (in seconds) are indicated in the panel. 


\section{Bibliography}

[1] H. A. Barnes. Thixotropy - a review. Journal of Non-Newtonian fluid mechanics, 70(1):1-33, 1997.

[2] E. Barsoukov and J. R. Macdonald. Impedance spectroscopy: theory, experiment, and applications. John Wiley \& Sons, 2005.

[3] M. Cerbelaud, B. Lestriez, R. Ferrando, A. Videcoq, M. Richard-Plouet, M. T. Caldes, and D. Guyomard. Numerical and experimental study of suspensions containing carbon blacks used as conductive additives in composite electrodes for lithium batteries. Langmuir, 30(10):2660-2669, 2014.

[4] H. N. Chen and Y. C. Lu. A high-energy-density multiple redox semi-solidliquid flow battery. Advanced Energy Materials, 6(8), 2016.

[5] R. Derooij, A. A. Potanin, D. Vandenende, and J. Mellema. Steady shear viscosity of weakly aggregating polystyrene latex dispersions. Journal of Chemical Physics, 99(11):9213-9223, 1993.

[6] R. Derooij, A. A. Potanin, D. Vandenende, and J. Mellema. Rheological behavior of weakly aggregating colloids - viscosity of the structure formed in a steady shear-flow. Colloid Journal, 56(4):476-486, 1994.

[7] J.-B. Donnet. Carbon black: science and technology. CRC Press, 1993.

[8] Y. R. Dougassa, J. Jacquemin, L. El Ouatani, C. Tessier, and M. Anouti. Viscosity and carbon dioxide solubility for lipf6, litfsi, and lifap in alkyl carbonates: lithium salt nature and concentration effect. The Journal of Physical Chemistry B, 118(14):3973-3980, 2014.

[9] M. Duduta, B. Ho, V. C. Wood, P. Limthongkul, V. E. Brunini, W. C. Carter, and Y. M. Chiang. Semi-solid lithium rechargeable flow battery. Advanced Energy Materials, 1(4):511-516, 2011.

[10] K. Dullaert and J. Mewis. Stress jumps on weakly flocculated dispersions: Steady state and transient results. Journal of colloid and interface science, 287(2):542-551, 2005.

[11] F. Y. Fan, W. H. Woodford, Z. Li, N. Baram, K. C. Smith, A. Helal, G. H. McKinley, W. C. Carter, and Y. M. Chiang. Polysulfide flow batteries enabled by percolating nanoscale conductor networks. Nano Letters, 14(4):2210-2218, 2014.

[12] D. B. Genovese. Shear rheology of hard-sphere, dispersed, and aggregated suspensions, and filler-matrix composites. Advances in colloid and interface science, 171:1-16, 2012.

[13] S. Hamelet, D. Larcher, L. Dupont, and J. M. Tarascon. Silicon-based non aqueous anolyte for li redox-flow batteries. Journal of the Electrochemical Society, 160(3):A516-A520, 2013.

[14] S. Hamelet, T. Tzedakis, J. B. Leriche, S. Sailler, D. Larcher, P. L. Taberna, P. Simon, and J. M. Tarascona. Non-aqueous li-based redox flow batteries. 
Journal of the Electrochemical Society, 159(8):A1360-A1367, 2012.

[15] T. Hao and R. E. Riman. Calculation of interparticle spacing in colloidal systems. Journal of colloid and interface science, 297(1):374-377, 2006.

[16] A. Helal, T. Divoux, and G. H. McKinley. Simultaneous rheo-electric measurements of strongly conductive complex fluids. arXiv preprint arXiv:1604.00336, 2016.

[17] J. Jamnik and J. Maier. Treatment of the impedance of mixed conductors equivalent circuit model and explicit approximate solutions. Journal of The Electrochemical Society, 146(11):4183-4188, 1999.

[18] M. Kawaguchi, M. Okuno, and T. Kato. Rheological properties of carbon black suspensions in a silicone oil. Langmuir, 17(20):6041-6044, 2001.

[19] S. Kirkpatrick. Classical transport in disordered media: scaling and effectivemedium theories. Physical Review Letters, 27(25):1722, 1971.

[20] C. Kruif, E. Van Iersel, A. Vrij, and W. Russel. Hard sphere colloidal dispersions: Viscosity as a function of shear rate and volume fraction. The Journal of chemical physics, 83(9):4717-4725, 1985.

[21] Z. Li, K. C. Smith, Y. J. Dong, N. Baram, F. Y. Fan, J. Xie, P. Limthongkul, W. C. Carter, and Y. M. Chiang. Aqueous semi-solid flow cell: demonstration and analysis. Physical Chemistry Chemical Physics, 15(38):1583315839, 2013.

[22] L. Madec, M. Youssry, M. Cerbelaud, P. Soudan, D. Guyomard, and B. Lestriez. Surfactant for enhanced rheological, electrical, and electrochemical performance of suspensions for semisolid redox flow batteries and supercapacitors. ChemPlusChem, 80(2):396-401, 2015.

[23] A. Medalia and E. Hagopian. Rheology of dispersant-free aqueous slurries of carbon black. Rheologica Acta, 3(2):100-111, 1963.

[24] J. Mewis. Thixotropy-a general review. Journal of Non-Newtonian Fluid Mechanics, 6(1):1-20, 1979.

[25] J. Mewis and N. J. Wagner. Thixotropy. Advances in Colloid and Interface Science, 147:214-227, 2009.

[26] J. Mewis and N. J. Wagner. Colloidal suspension rheology. Cambridge University Press, 2012.

[27] C. O. Osuji, C. Kim, and D. A. Weitz. Shear thickening and scaling of the elastic modulus in a fractal colloidal system with attractive interactions. Physical Review E, 77(6), 2008.

[28] C. O. Osuji and D. A. Weitz. Highly anisotropic vorticity aligned structures in a shear thickening attractive colloidal system. Soft Matter, 4(7):13881392, 2008.

[29] G. Ovarlez, L. Tocquer, F. Bertrand, and P. Coussot. Rheopexy and tunable yield stress of carbon black suspensions. Soft Matter, 9(23):5540-5549, 2013. 
[30] A. Potanin. Thixotropy and rheopexy of aggregated dispersions with wetting polymer. Journal of Rheology (1978-present), 48(6):1279-1293, 2004.

[31] A. Potanin, R. De Rooij, D. Van den Ende, and J. Mellema. Microrheological modeling of weakly aggregated dispersions. The Journal of chemical physics, 102(14):5845-5853, 1995.

[32] A. A. Potanin, R. Derooij, D. Vandenende, and J. Mellema. Microrheological modeling of weakly aggregated dispersions. Journal of Chemical Physics, 102(14):5845-5853, 1995.

[33] E. H. Purnomo, D. van den Ende, J. Mellema, and F. Mugele. Rheological properties of aging thermosensitive suspensions. Physical Review E, 76(2):021404, 2007.

[34] J. J. Richards, A. D. Scherbarth, N. J. Wagner, and P. D. Butler. Mixed ionic/electronic conducting surface layers adsorbed on colloidal silica for flow battery applications. ACS Applied Materials \& Interfaces, 8(36):2408924096, 2016.

[35] C. J. Rueb and C. F. Zukoski. Viscoelastic properties of colloidal gels. Journal of Rheology, 41(2):197-218, 1997.

[36] G. Schramm. A practical approach to rheology and rheometry. Haake Karlsruhe, 1994.

[37] P. Sheng, E. Sichel, and J. Gittleman. Fluctuation-induced tunneling conduction in carbon-polyvinylchloride composites. Physical Review Letters, 40(18):1197, 1978.

[38] R. Sherman, L. Middleman, and S. Jacobs. Electron transport processes in conductor-filled polymers. Polymer Engineering \& Science, 23(1):36-46, 1983.

[39] E. K. Sichel, J. I. Gittleman, and P. Sheng. Transport properties of the composite material carbon-poly(vinyl chloride). Physical Review B, 18(10):57125716, 1978.

[40] K. C. Smith, Y. M. Chiang, and W. C. Carter. Maximizing energetic efficiency in flow batteries utilizing non-newtonian fluids. Journal of the Electrochemical Society, 161(4):A486-A496, 2014.

[41] T. F. Tadros. Rheology of dispersions: principles and applications. John Wiley \& Sons, 2011.

[42] V. A. Tolpekin, M. H. G. Duits, D. van den Ende, and J. Mellema. Aggregation and breakup of colloidal particle aggregates in shear flow, studied with video microscopy. Langmuir, 20(7):2614-2627, 2004.

[43] T. Vanvliet, H. J. M. Vandijk, P. Zoon, and P. Walstra. Relation between syneresis and rheological properties of particle gels. Colloid and Polymer Science, 269(6):620-627, 1991.

[44] E. Ventosa, D. Buchholz, S. Klink, C. Flox, L. G. Chagas, C. Vaalma, 
W. Schuhmann, S. Passerini, and J. R. Morante. Non-aqueous semi-solid flow battery based on na-ion chemistry. p2-type naxni0.22co0.11mn0.66o2nati2(po4)(3). Chemical Communications, 51(34):7298-7301, 2015.

[45] W. Wolthers, M. Duits, D. Van Den Ende, and J. Mellema. Shear history dependence of the viscosity of aggregated colloidal dispersions. Journal of Rheology (1978-present), 40(5):799-811, 1996.

[46] W. Wolthers, D. Van den Ende, M. Duits, and J. Mellema. The viscosity and sedimentation of aggregating colloidal dispersions in a couette flow. Journal of Rheology (1978-present), 40(1):55-67, 1996.

[47] M. Youssry, L. Madec, P. Soudan, M. Cerbelaud, D. Guyomard, and B. Lestriez. Non-aqueous carbon black suspensions for lithium-based redox flow batteries: rheology and simultaneous rheo-electrical behavior. Physical Chemistry Chemical Physics, 15(34):14476-14486, 2013.

[48] M. Youssry, L. Madec, P. Soudan, M. Cerbelaud, D. Guyomard, and B. Lestriez. Formulation of flowable anolyte for redox flow batteries: Rheoelectrical study. Journal of Power Sources, 274:424-431, 2015.

[49] Y. Zhang, A. Narayanan, F. Mugele, M. C. Stuart, and M. H. G. Duits. Charge inversion and colloidal stability of carbon black in battery electrolyte solutions. Colloids and Surfaces a-Physicochemical and Engineering Aspects, 489:461-468, 2016. (Chapter 2 of this thesis). 



\title{
Chapter 4
}

\section{Cycling-Rheoimpedance}

\section{Device}

\author{
Aditya Narayanan, Frieder Mugele, Michel Duits
}

The research described in this Chapter has been published as:

Device for rheometry, impedance spectroscopy, and electrochemistry on fluid electrodes, Review of scientific instruments, 90(2):025112, 2019

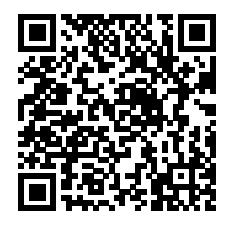




\subsection{Abstract}

We describe the extension of a rheometer to enable in-situ impedance spectroscopy and electrochemical cycling. Key advantages of this instrument over traditional flow-channel based methods for studying fluid electrodes, are the possibilities to monitor the rheological properties during cycling, as well as to control the mechanical history of the sample. We describe two electrochemical configurations of the instrument, allowing fluid electrodes to be studied as full and half-cells. To demonstrate the systems capabilities, we present characterizations of 4 different fluid electrode systems.

\subsection{Introduction}

Novel solutions for grid energy storage and production such as Semi Solid Flow Batteries $(\mathrm{SSFB})^{1}$, Flow Super-capacitors ${ }^{10}$ and Flow Capacitive Mixing Generators $^{9}$ are receiving a strong interest in current research and development ${ }^{2}$. Semi-solid flow architectures use fluid electrodes with active particles that store charge either through faradic or (pseudo) capacitive processes and conductive particles that impart electronic conductivity dispersed in an electrolyte solution. The conductive particles are typically colloidal carbon blacks. They transfer electrons through a percolated (agglomerate) network, which can even be formed during flow. In the quiescent state, these networks also prevent active particle sedimentation by providing a finite yield stress to the suspension ${ }^{6,7}$.

One major challenge that comes with the development of these systems lies in the dispersed state of the colloidal particles. In contrast to traditional batteries, a fluid electrode can adapt its structure to externally imposed fields such as gravity, 
mechanical shear or electrochemical potential. The response of the system can be a modification of the fluid structure without altering the particles themselves, but it can also take place via changes in the particles' surfaces and colloidal interactions. These changes at the particle and overall structure level can strongly influence the performance of the fluid electrode. Not only shear rate, but also shear history have been found to significantly change the electronic conductivity and rheological properties of the electrodes, due to a restructuring of particle agglomerates ${ }^{6,8,14}$. Electrochemical operation of SSFB fluids was shown to induce surface layers such as SEI on the particles, thereby strongly affecting the low frequency electrical impedance of the fluid ${ }^{7,13}$. In Flow Capacitive Mixing electrodes, electrochemically induced changes in salt concentration ${ }^{2}$ can affect colloidal interactions and hence the agglomeration behavior ${ }^{16}$ and microstructure.

Each of these examples underlines that the understanding (and subsequent performance optimization) of colloidal fluid electrodes, requires the ability to control both the mechanical and electric fields, as well as the availability of in-situ characterization tools. At the minimum, two different types of electrochemical sensing and control are needed: the ability to apply a DC bias for electrochemical cycling, and a small AC perturbation for Impedance Spectroscopy, both on suitable counter electrodes. Besides that, the rheological capabilities should include at least measurements of the yield stress and viscosity flow curve. Such versatility cannot be offered by existing instruments; flow-channel setups capable of electrochemical testing ${ }^{1,4,5,10,13}$ do not allow a well-defined control over mechanical history, while existing combined rheology-impedance spectroscopy instruments ${ }^{3,6,11,12,14,15}$ do not allow electrochemical cycling. 
In this paper, we describe a novel setup capable of simultaneous rheometry, impedance spectroscopy and electrochemical cycling. For electrochemical cycling two (counter) electrode configurations are described. The first, applicable to Semi Solid Flow Battery electrodes uses a lithium foil as a counter electrode. The second uses a larger volume of a second fluid as the counter electrode. This configuration is particularly suited for studying Flow Super Capacitor electrodes that require a very high surface area counter-electrode, and other flow systems in a full cell configuration.

\subsection{Global Setup}

Figure 4.1 shows a schematic of the apparatus. It consists of a parallel plate geometry for rheometry with both shearing surfaces (CC1 and CC2) acting also as current collectors for impedance spectroscopy. This geometry was chosen to achieve a uniform electric field across the sample (FE1), at the expense of a constant strain field. The rheometer applies torques and measures strains on the upper geometry.

The bottom stationary shearing surface (CC2) is a perforated disk that allows the fluid electrode to interact with a counter electrode below, which is used for electrochemical cycling. A polymeric membrane that is permeable to solvent and ions but not to the colloidal particles, separates the two electrodes. Depending on the application, two counter electrode configurations are possible. The first is the Semi-Solid Flow Battery half-cell which uses a lithium metal foil as the counter electrode. The second configuration is the Flow Supercapacitor / Semi Solid Flow Battery full cell which uses a second fluid as a counter electrode. In 


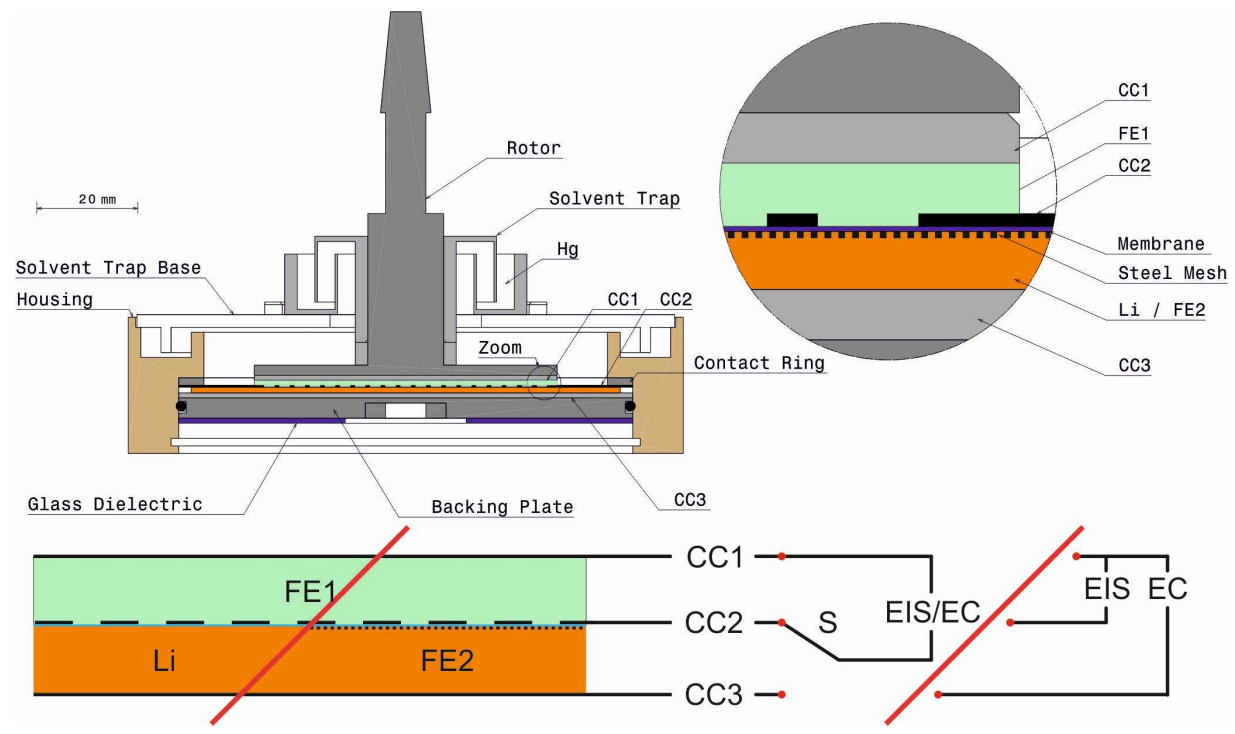

Figure 4.1: Top: Cross section of the apparatus. The encircled inset shows a zoom-in. Bottom: Schematic of the measurement geometry and external electric circuit in two configurations, separated by a slash: the LHS corresponds to a halfcell, the RHS to a full cell. Meaning of symbols:

CC1 upper current collector and shearing surface (grey)

CC2 current collector and lower shearing surface (black)

CC3 lower current collector (light grey)

EC electrochemical cycling

EIS electrical impedance spectroscopy

FE1 fluid electrode 1 (green)

FE2 fluid electrode 2 (orange)

$\mathrm{Li} \quad$ lithium foil (orange)

S switch

either case, the fluid electrode under study (between $\mathrm{CC} 1$ and $\mathrm{CC} 2$ ) is in direct contact with two ionically blocking but electronically reversible metal current collectors. This configuration is particularly advantageous for impedance measurements as it allows a direct probing of the upper fluid electrode's electronic 
resistance (besides some interfacial contributions); i.e. the low frequency limit of the real impedance ${ }^{6,7}$.

\subsubsection{Detailed Description}

A Haake Rheostress RS 600 (geometrically equivalent to the RS 6000 model) controlled stress rheometer (not shown), forms the base and frame of the instrument. The upper rheometry geometry is a combination of a rotating body belonging to the rheometer (a 'rotor') and a disk. The former is a commercial (Haake) stainless steel electro-rheology rotor with a ceramic inter-connect to provide electrical isolation from the rheometer spindle. To the bottom of the rotor, a $60 \mathrm{~mm}$ stainless steel disk (CC1) was glued using V-Blocks for alignment. A very small amount of (non-conductive) cyanoacrylate superglue was used to achieve a strong bonding between the rotor and disk, while preserving electrical contact. To electrically connect this upper rheometry geometry to the outside, with minimal electrical noise and mechanical resistance, a steel solvent trap is used with mercury as a working fluid. The variation in impedance of the mercury connection due to rotation, was measured to be less than $10 \mathrm{~m} \Omega$. The contribution to the measured torque was negligible for all measurements to be shown. The stationary part of the solvent trap is placed on a splittable $3 \mathrm{D}$ printed solvent trap base that sits on a polypropylene housing.

The subsequent parts are assembled into the polypropylene housing which is placed on the base of the rheometer. A thin stainless-steel disk with $(\sim 1 \mathrm{~mm}$ radius) perforations (CC2) is used as the bottom shearing surface. It rests on a metal contact ring which is electrically connected to the outside via a screw. A permeable polymeric membrane is placed below the perforated disk to elec- 
tronically separate the (upper) fluid electrode FE1 from the lower components (including the counter electrode).

The placement of the lower components depends on the configuration. For the Semi-Solid Flow Battery half-cell configuration, a lithium foil disk is placed below the membrane on top of a third current collector plate (CC3). A thick lithium disk is used to give the membrane mechanical support. For the Flow Supercapacitor/Semi Solid Flow Battery full cell configuration, a stainless-steel mesh is placed below the membrane to provide mechanical support. A spacer (not shown) and the third current collector along with the mesh create a compartment that is filled with the second fluid electrode (FE2).

A metal backing plate with an O-ring seals the cell and is electrically connected to the outside via a screw. The backing plate is also used to align the lower rheometry geometry with the rheometer base and the spindle, using an alignment pin. Below the backing plate is a thin glass disk that acts as a dielectric, isolating the counter electrode from the rheometer body. The relatively high thermal conductivity of the glass compared to other dielectrics allows for effective temperature measurement and control from the rheometer base. A retainer spring (not shown) in a groove of the polypropylene housing presses it and all the lower geometry parts onto the rheometer base.

There are two possible configurations for electrochemistry and impedance spectroscopy that can be used with the instrument; single and dual potentiostat. The single potentiostat configuration uses relays to switch between electrochemical measurement and control. During electrochemical cycling switch S is 'down' and the potential of $\mathrm{CC} 1$ (and the fluid electrode) is controlled versus CC3, while $\mathrm{CC} 2$ is left floating. During spectroscopic measurements switch $\mathrm{S}$ is 'up' and 
the impedance spectra are measured between CC1 and CC2 with CC3 floating. The second dual potentiostat configuration allows simultaneous impedance measurement and electrochemical control. One potentiostat controls the potential of CC2 versus CC1 for impedance spectroscopy and measures the currents through it. The second does the same for CC3 (versus CC1). It is important that the potentiostat in control of CC3 has a lower bandwidth than the AC impedance measurement, to prevent cross feedback. Thus, the impedance measurement is typically limited to higher frequencies unless the potentiostat connected to CC3 is set to open circuit.

\subsubsection{Assembly and Sample loading}

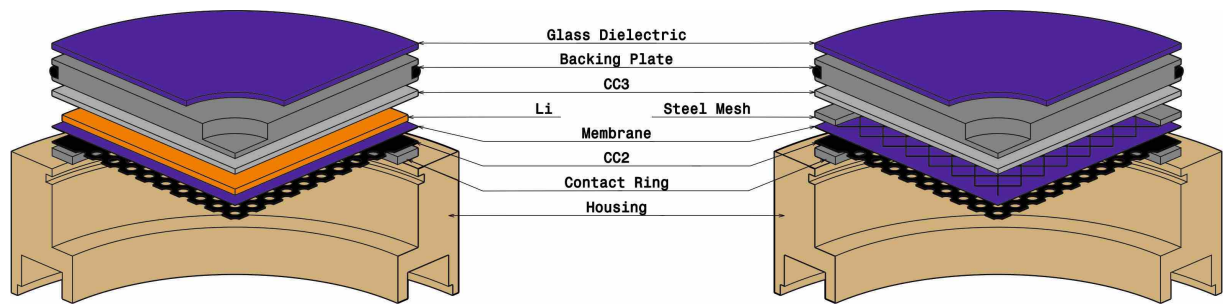

Figure 4.2: Sections demonstrating the assembly of the device in (left) half-cell and (right) full-cell configurations.

Fig. 4.2 Sections demonstrating the assembly of the device in (left) half-cell and (right) full-cell configurations. The lower rheometry geometry is first assembled, in a top down manner, into the inverted polypropylene base as shown in Fig 4.2. After the contact ring, perforated plate and solvent wetted membrane are placed, the counter electrode is assembled. In the case of the SSFB half-cell, the lithium foil disk and then the third current collector are placed. For the SSFB/FSC fullcell, the steel mesh with spacer is placed. A side screw (not shown) is used to 
hold the pressed spacer and mesh against the membrane, flattening it. An excess of the fluid electrode 2 (not shown) is introduced and the third current collector is pressed onto the spacer. Excess slurry is then wiped off. For both configurations, the backing plate is then pressed against the third current collector. The glass dielectric and retaining spring (not shown) are placed and the bottom geometry is installed on the rheometer base. The rotor is then screwed on the rheometer spindle.

After zeroing the rheometer gap, the sample (fluid electrode 1) is introduced. The gap is closed, excess sample is trimmed off, and the solvent trap is assembled and filled with mercury.

\subsubsection{Rheological Verification}

The modified measurement geometry was verified using two viscosity standards at various settings for the gap, i.e. the distance between $\mathrm{CC} 1$ and $\mathrm{CC} 2$. For all gaps, the calibration oils gave viscosities that remained constant within $5 \%$ (standard deviation) as the shear rate was varied (logarithmically) from 10 to $1000 \mathrm{~s}^{-1}$. Figure 4.3 shows the (shear-rate averaged) viscosity as a function of the gap height; the variation is less than $10 \%$. These results are similar to those obtained with the standard bottom plate of the instrument.

\subsection{Application to flow systems}

We demonstrate the versatility of the instrument by presenting the evolution of the fluid yield stress and Low Frequency Real Impedance (LFRI) on electrochemical cycling for four fluid electrode systems. We directly present the LFRI over a 


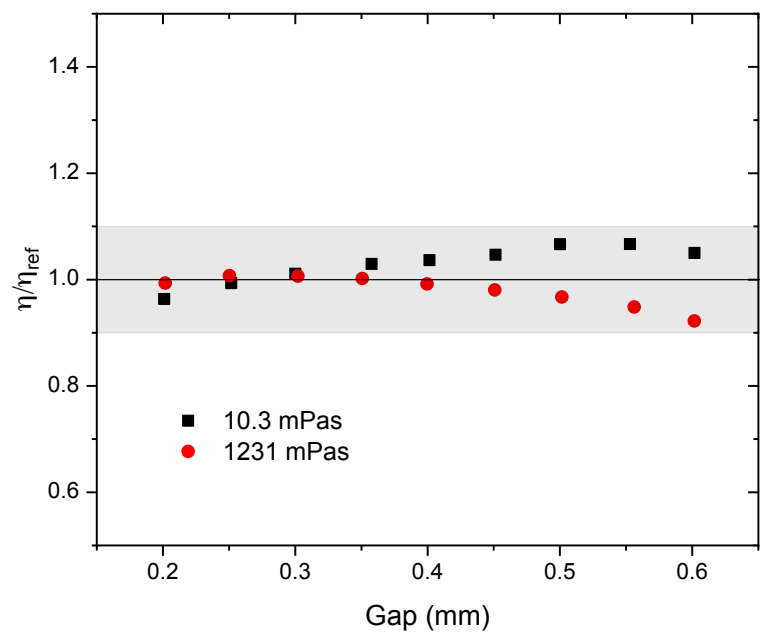

Figure 4.3: Viscosity versus plate-plate gap for two calibration oils. The black lines are the reference viscosities. The grey bars represent a $10 \%$ error.

fit electronic resistance as it does not rely on models of the EIS spectra (shown in SI) which can vary with system and charge state. We have previously demonstrated $^{7}$ that the LFRI is a reasonable measure of the trends in the electronic resistance.

\subsubsection{SSFB fluid electrodes in half-cell configuration}

Fig. 4.4 shows the evolution of the yield stress and Low Frequency Real Impedance (LFRI) of a SSFB anolyte (Lithium Titanate; LTO based) and catholyte (Lithium Nickel Cobalt Manganese oxide; LiNCM based) before and during electrochemical cycling. Details of the electrode materials and preparation are given in the SI. To demonstrate the importance of mechanical pre-shear (and subsequent rest), 

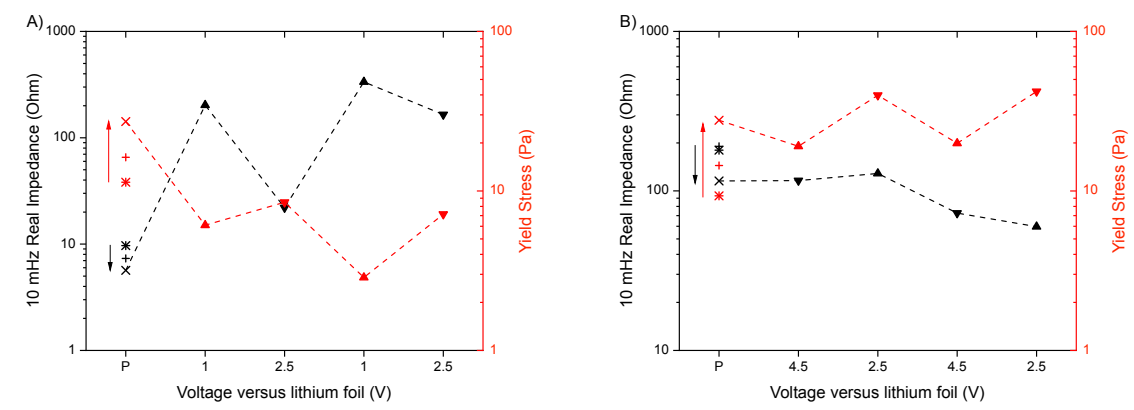

Figure 4.4: Low frequency real impedance and Yield stress of anolyte (A) and catholyte $(B)$ versus charge state, where $P$ indicates the pristine state, and the other states are defined via the reached voltage. Arrows mark increasing pre-shear rates $\left(100,316,1000 \mathrm{~s}^{-1}\right)$ for the pristine state. See SI (Fig. 4.7) for impedance spectra.

we first consider the samples in the electrochemically pristine state. We applied a pre-shear at the indicated rate for $200 \mathrm{~s}$ and allowed a rest time of $200 \mathrm{~s}$. For both anolyte (Fig. 4.4 A) and catholyte (Fig. 4.4 B), it is observed that a higher pre-shear rate leads to a higher yield stress and a lower LFRI. Rates higher than $1000 \mathrm{~s}^{-1}$ do not lead to a significant enhancement of the effect. This indicates a complete breakup of the carbon black network, before allowing the primary units to agglomerate again during the subsequent rest ${ }^{3,6}$, thereby defining a re-

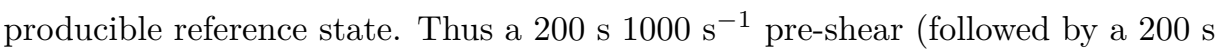
rest) was applied before all measurements.

In subsequent electrochemical cycling experiments, the single potentiostat configuration was used. Each fluid electrode (FE1) was cycled in the half cell configuration versus a lithium foil in separate experiments. Experimental details hereof are included in the SI. For the anolyte, the low frequency real impedance shows 
very strong alternations with the state of charge. Additionally, there is an increase in LFRI per (charged or discharged) state, when electrochemically cycled. The yield stress also shows strong alternations in the opposite direction, with a lower yield stress correlating to a higher impedance and vice versa. Cycling the catholyte also has significant effects with an increased yield stress and lowered impedance in the charged state. The effects on the catholyte are however much weaker than those of the anolyte. The increases in the impedance and decreases in yield stress (observed within the accepted operating range of the materials ${ }^{2,13}$ ) are very detrimental to SSFB fluid electrodes. The observed trends can be partially explained by formation of surface layers (Solid Electrolyte Interface) on the conductive carbons during charging, that partially dissolve on discharging ${ }^{7}$. It is of note that the effect of different pre-shears on the pristine electrode yield stress and LFRI is similar in magnitude to that of a full charge discharge cycle. This highlights the importance of a well-defined mechanical history to fluid electrode measurements.

\subsubsection{FSC fluid electrode in full-cell configuration}

Fig. 5 shows the evolution of the yield stress and LFRI of a non-aqueous (propylene carbonate based) and an aqueous flow super capacitor fluid electrode when electrochemically cycled. The fluid electrodes contain conductive carbon and no electrochemically active particles. Details of the electrode materials and preparation are given in the SI. The counter electrode in both cases is a much larger volume ( 4 times) of the same fluid electrode. The fluid electrodes under study were pre-sheared at $1000 \mathrm{~s}^{-1}$ for $200 \mathrm{~s}$ followed by a rest time of $200 \mathrm{~s}$ before all measurements. 

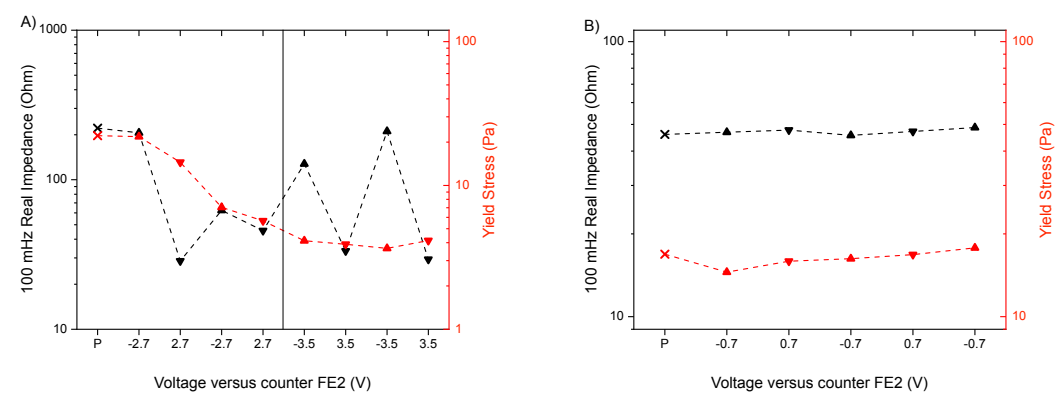

Figure 4.5: Evolution of the LFRI and Yield stress of a non-aqueous (A) and aqueous (B) flow super capacitor fluid electrode (without electroactive particles) when cycled. In the non-aqueous case, the vertical line demarcates two voltage regimes. See SI (Fig. 4.8, 4.9) for impedance spectra.

During electrochemical cycling experiments, the dual potentiostat configuration was used. The non-aqueous fluid electrode's LFRI shows strong alternations, with the negative potential state consistently presenting higher LFRI values. The alternations diminish with cycle number in the $2.7 \mathrm{~V}$ window and increase in the $3.5 \mathrm{~V}$ one. In all cases however the LFRI is actually lower than that of the pristine state. The yield stress does not show any correlation with the LFRI. Instead it decreases every half cycle up to some lower limit. The aqueous fluid electrode on the other hand does not exhibit any significant effect of electrochemical cycling. As these systems do not show detrimental effects (and even show somewhat positive ones), they may be viable candidates for FSC electrodes.

\subsection{Summary}

We have described a modification to a (typical) commercial rheometer that extends its measurement capabilities for colloidal suspensions, to in situ impedance 
spectroscopy and electrochemical cycling. Example experiments firstly confirm the importance of controlled pre-shear in order to define a reproducible reference state, and secondly show strong effects of electrochemical cycling on the fluid electrodes' electrical and mechanical properties. This highlights the usefulness and relevance of the setup to study suspension fluid electrodes. As the apparatus can be set up with a modest amount of effort on commercial rheometers, we expect it to be of use to others involved in fluid electrode research. We have provided downloadable CAD files of all the parts online. 


\subsection{Supplementary Information}

\subsubsection{Materials and sample preparation}

Ethylene carbonate (EC), dimethyl carbonate (DMC) and propylene carbonate (PC) were obtained from Sigma Aldrich (anhydrous, 99\%+ purity). Binary mixtures of EC and DMC were 1:1 by volume. LP30 (EC:DMC 1:1 with 1M LiPF 6 ) was obtained from BASF. Ketjen Black EC 600JD powder (KB) was obtained from AkzoNobel. LTO, $\mathrm{Li}_{4} \mathrm{Ti}_{5} \mathrm{O}_{12}$ powder was obtained from Südchemie. LiNCM powder was provided by the Helmholtz Institute, Karlruhe Institute of Technology. $\mathrm{LiPF}_{6}$ was obtained from Alfa Aesar. $\mathrm{Na}_{2} \mathrm{SO}_{4}$ and $\mathrm{TEA}-\mathrm{BF}_{4}$ were purchased from Sigma Aldrich. Lithium foil was purchased from Alfa Aesar (99.9\%). Sample preparations and experiments for SSFB fluid electrodes were carried out in an MBraun Argon-filled glove box $\left(\mathrm{O}_{2}, \mathrm{H}_{2} \mathrm{O}\right.$ below 1 ppm). The composition of the electrodes is given in table 4.1. For the SSFB fluid electrodes, the dry particles were first wetted by solvent. Afterwards, salt was added via a concentrated solution (as in ref. [1]). For other electrodes, salt solutions were directly added to the dry particles. The samples were then homogenized by rotor stator mixing (Ultraturrax) at $15000 \mathrm{rpm}$ for $2 \mathrm{~min}$, and loaded in the rheo-impedance setup.

\subsubsection{Electrochemical measurements and control}

For the single potentiostat configuration, a Zurich Instruments HF2IS impedance spectroscope was used in a four-terminal mode for electrochemical control and impedance measurements (as in ref. ${ }^{7}$ ). For the dual potentiostat configuration, a Biologic VSP-300 was used. Custom LabVIEW programs were used to synchronize the spectroscope / potentiostat and rheometer. Impedance spectroscopy 


\begin{tabular}{lllll}
$\begin{array}{l}\text { Fluid } \\
\text { Electrode }\end{array}$ & Solvent & Electrolyte & Carbon & Active particle \\
\hline $\begin{array}{l}\text { SSFB } \\
\text { Anolyte }\end{array}$ & EC:DMC 1:1 (v:v) & $1 \mathrm{M} \mathrm{LiPF} 6$ & $1 \% \mathrm{~KB}$ & $5 \% \mathrm{LTO}$ \\
$\begin{array}{l}\text { SSFB } \\
\text { Catholyte }\end{array}$ & EC:DMC 1:1 (v:v) & $1 \mathrm{M} \mathrm{LiPF}_{6}$ & $1 \% \mathrm{~KB}$ & $5 \%$ LiNCM \\
$\begin{array}{l}\text { SFC } \\
\text { aqueous }\end{array}$ & milliQ water & $1 \mathrm{M} \mathrm{Na}_{2} \mathrm{SO}_{4}$ & $2 \% \mathrm{~KB}$ & None \\
$\begin{array}{l}\text { SFC non- } \\
\text { aqueous }\end{array}$ & $\mathrm{PC}$ & $1 \mathrm{M} \mathrm{TEA}-\mathrm{BF}_{4}$ & $1.5 \% \mathrm{~KB}$ & None
\end{tabular}

Table 4.1: Composition of fluid electrodes used. All percentages are $w / w$.

measurements were made with an amplitude of $50 \mathrm{mV}$.

\subsubsection{Rheoimpedance protocol}

SSFB electrodes were (dis-) charged galvanostatically using a current of $1.5 \mathrm{~mA}$. All other electrodes were (dis-) charged using a twenty minute potentiostatic step. Thereafter EIS measurements were performed, taking into account the mechanical pre-shear protocol mentioned in the main text. Next the yield stress was measured (Fig. 4.6) by ramping up the shear stress (62 s per stress decade) while measuring the strain. The $\log ($ strain) versus $\log$ (stress) curve was fitted with two straight lines and the stress at the intersection was taken to be the yield stress (as in ref. ${ }^{6}$ ). 


\subsubsection{Stress Strain Curves (Yield Stress)}

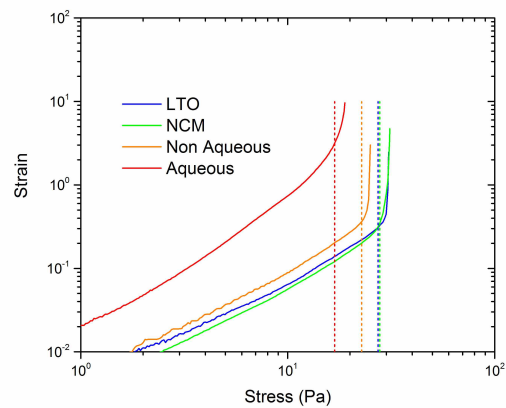

Figure 4.6: Measured strain versus applied stress of the various fluid electrodes, in the pristine state. The dashed vertical lines indicate the extracted yield stress.

\subsubsection{Impedance Spectra}
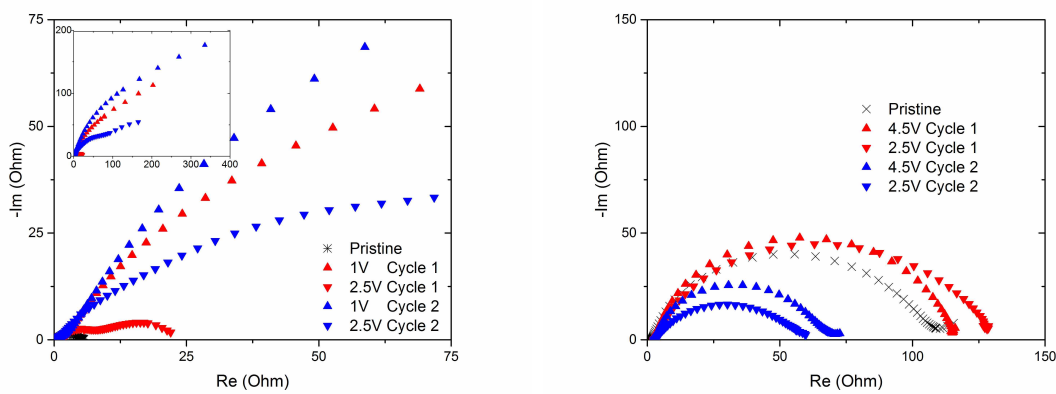

Figure 4.7: Nyquist plots of the impedance spectra of SSFB anolyte (Left) and catholyte (Right) when cycled. 

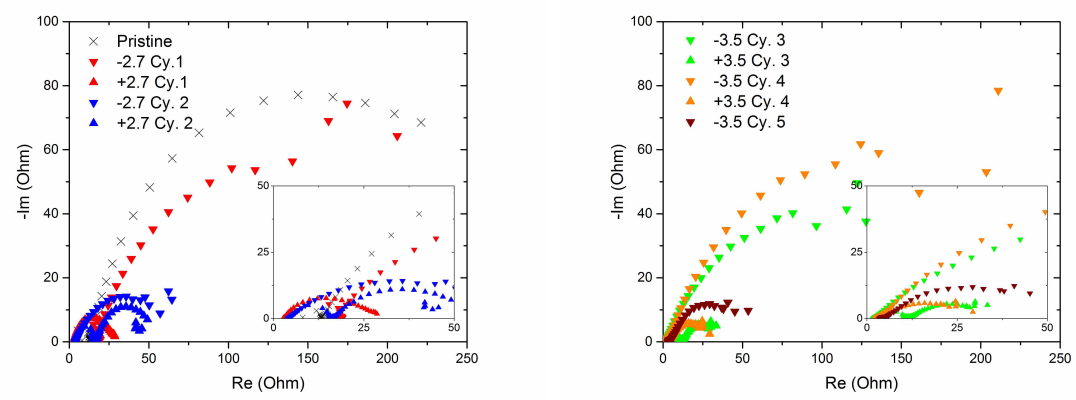

Figure 4.8: Nyquist plots of the impedance spectra of a non-aqueous flow super capacitor fluid electrode when cycled. Left: Initial cycles Right: Subsequent cycles.

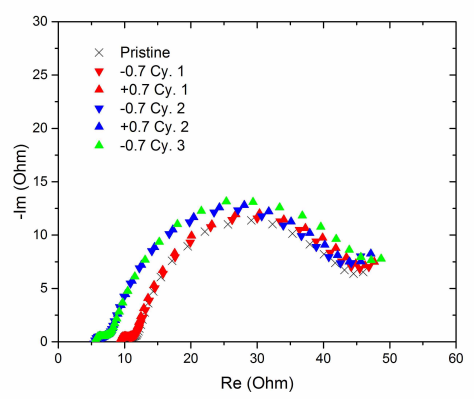

Figure 4.9: Nyquist plots of the impedance spectra of an aqueous flow super capacitor fluid electrode when cycled. 


\section{Bibliography}

[1] M. Duduta, B. Ho, V. C. Wood, P. Limthongkul, V. E. Brunini, W. C. Carter, and Y. M. Chiang. Semi-solid lithium rechargeable flow battery. Advanced Energy Materials, 1(4):511-516, 2011.

[2] K. B. Hatzell, M. Boota, and Y. Gogotsi. Materials for suspension (semisolid) electrodes for energy and water technologies. Chemical Society Reviews, 44(23):8664-8687, 2015.

[3] A. Helal, T. Divoux, and G. H. McKinley. Simultaneous rheo-electric measurements of strongly conductive complex fluids. arXiv preprint arXiv:1604.00336, 2016.

[4] S.-i. Jeon, H.-r. Park, J.-g. Yeo, S. Yang, C. H. Cho, M. H. Han, and D. K. Kim. Desalination via a new membrane capacitive deionization process utilizing flow-electrodes. Energy \& Environmental Science, 6(5):1471-1475, 2013.

[5] Z. Li, K. C. Smith, Y. Dong, N. Baram, F. Y. Fan, J. Xie, P. Limthongkul, W. C. Carter, and Y.-M. Chiang. Aqueous semi-solid flow cell: demonstration and analysis. Physical Chemistry Chemical Physics, 15(38):1583315839, 2013.

[6] A. Narayanan, F. Mugele, and M. H. G. Duits. Mechanical history dependence in carbon black suspensions for flow batteries: a rheo-impedance study. Langmuir, 2017. (Chapter 3 of this thesis).

[7] A. Narayanan, D. Wijnperlé, F. Mugele, D. Buchholz, C. Vaalma, X. Dou, S. Passerini, and M. Duits. Influence of electrochemical cycling on the rheoimpedance of anolytes for li-based semi solid flow batteries. Electrochimica Acta, 251:388-395, 2017. (Chapter 5 of this thesis).

[8] G. Ovarlez, L. Tocquer, F. Bertrand, and P. Coussot. Rheopexy and tunable yield stress of carbon black suspensions. Soft Matter, 9(23):5540-5549, 2013.

[9] S. Porada, D. Weingarth, H. Hamelers, M. Bryjak, V. Presser, and P. Biesheuvel. Carbon flow electrodes for continuous operation of capacitive deionization and capacitive mixing energy generation. Journal of Materials Chemistry A, 2(24):9313-9321, 2014.

[10] V. Presser, C. R. Dennison, J. Campos, K. W. Knehr, E. C. Kumbur, and Y. Gogotsi. The electrochemical flow capacitor: a new concept for rapid energy storage and recovery. Advanced Energy Materials, 2(7):895-902, 2012.

[11] J. J. Richards, J. B. Hipp, J. K. Riley, N. J. Wagner, and P. D. Butler. Clustering and percolation in suspensions of carbon black. Langmuir, 2017.

[12] J. J. Richards, N. J. Wagner, and P. D. Butler. A strain-controlled rheosans instrument for the measurement of the microstructural, electrical, and mechanical properties of soft materials. Review of Scientific Instruments, 88(10):105115, 2017. 
[13] E. Ventosa, G. Zampardi, C. Flox, F. La Mantia, W. Schuhmann, and J. Morante. Solid electrolyte interphase in semi-solid flow batteries: a wolf in sheep's clothing. Chemical Communications, 51(81):14973-14976, 2015.

[14] M. Youssry, L. Madec, P. Soudan, M. Cerbelaud, D. Guyomard, and B. Lestriez. Non-aqueous carbon black suspensions for lithium-based redox flow batteries: rheology and simultaneous rheo-electrical behavior. Physical Chemistry Chemical Physics, 15(34):14476-14486, 2013.

[15] M. Youssry, L. Madec, P. Soudan, M. Cerbelaud, D. Guyomard, and B. Lestriez. Formulation of flowable anolyte for redox flow batteries: Rheoelectrical study. Journal of Power Sources, 274:424-431, 2015.

[16] Y. Zhang, A. Narayanan, F. Mugele, M. A. C. Stuart, and M. H. Duits. Charge inversion and colloidal stability of carbon black in battery electrolyte solutions. Colloids and surfaces A: Physicochemical and engineering aspects, 489:461-468, 2016. (Chapter 2 of this thesis). 


\title{
Chapter 5
}

\section{Electrochemical history}

\section{dependence}

\author{
Aditya Narayanan, Daniel Wijnperlé, Frieder Mugele, \\ Daniel Buchholz, Christoph Vaalmaa, Xinwei Dou, Ste- \\ fano Passerini, Michel Duits
}

The research described in this Chapter has been published as:

Influence of electrochemical cycling on the rheo-impedance of anolytes for Li-based Semi Solid Flow Batteries, Electrochimica acta, 251:388-395, 2017

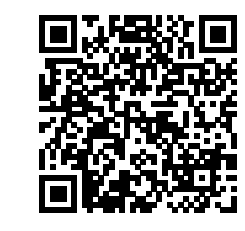




\subsection{Abstract}

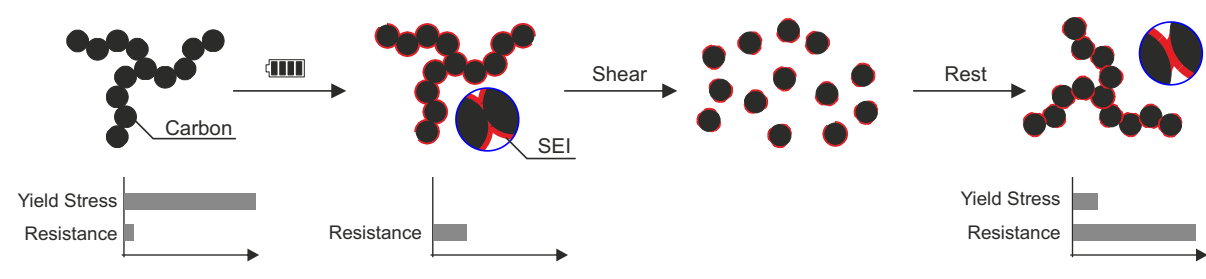

The recently launched concept of Semi-Solid Flow Batteries (SSFBs) shows a strong potential for flexible energy storage, but the liquid-dispersed state of the electrode materials introduces several aspects of which a scientific understanding is lacking. We studied the effect of electrochemical cycling on the rheological and electrical properties of a SSFB anolyte containing $\mathrm{Li}_{4} \mathrm{Ti}_{5} \mathrm{O}_{12}$ (LTO) and Ketjen Black (KB) particles in EC:DMC solvent with $1 \mathrm{M} \mathrm{LiPF}_{6}$, using an adapted rheometer that allows in situ electrochemical cycling and electrical impedance spectroscopy. Charging (lithiation) caused a reduction in the electronic conductivity, yield stress and high shear viscosity of the fluid electrode. For mildly reducing voltages $(1.4 \mathrm{~V})$, these changes were partially reversed on discharging. For more reducing voltages these changes were stronger and persistent. The finding of comparable trends for a fluid electrode without the LTO, lends support to a simplistic interpretation, in which all trends are ascribed to the formation of a surface layer around the conductive KB nanoparticles. This Solid Electrolyte Interphase (SEI) insulates particles and reduces the van der Waals attractions between them. SEI layers formed at less reducing voltages, partially dissolve during the subsequent discharge. Those formed at more reducing voltages, are thicker and permanent. As these layers increase the electronic resistance of the fluid electrode by (more than) an order of magnitude, our findings highlight sig- 
nificant challenges due to SEI formation that still need to be overcome to realize SSFBs.

\subsection{Introduction}

Semi-Solid Flow Batteries (SSFBs), as recently introduced by Duduta et $\mathrm{al}^{9}$, comprise a promising addition to the spectrum of rechargeable battery systems. The advantages of SSFBs over conventional batteries lie in the decoupling of power (cell size) and energy (tank size), and the potential for adjusting the chemistry of the system during operation. In particular non-aqueous SSFB systems are interesting, since they offer much higher energy densities as compared to more conventional aqueous redox flow systems ${ }^{9}$. The ability of non-aqueous SSFBs to provide and store energy in a flexible way makes them particularly promising for grid applications.

However, a key aspect in which SSFBs are yet unproven is their performance after repeated electrochemical cycling. While many SSFBs use the same materials $^{9,13}$ as conventional lithium-ion batteries, they may potentially degrade in different ways due to the dispersed state of the solid matter. In SSFBs (de)lithiation takes place in electrochemically active particles while the electrons are transported to the current collectors via conductive nanoparticles (CNPs). The occurrence of both particles in the (sub)micron size range has several consequences: i) the surface-to-volume ratio is relatively large, and ii) Brownian motion and interparticle interactions now play a role. Electrochemistry induced changes can therefore manifest themselves in different ways. They can directly affect individual particles (e.g. electronic conductivity, lithium uptake) but also collective 
effects are possible, because colloidal particles show a tendency for self-assembly into a microstructure. The colloidal interactions, which drive this assembly, are likely to be affected by the electrochemical cycling, and since the structure is kept dynamic by Brownian motion and/or shear flow, the microstructure may adapt to electrochemical changes.

While the precise microstructure of SSFB fluids is still to be ascertained, the generally accepted view ${ }^{9,13,19}$ is that in the absence of flow, the CNPs assemble into a branched percolating network. This network provides electronic conduction and sustains static forces, thereby resisting the sedimentation of particles. In flow, the network gets broken down into agglomerates with a size that depends on the shear rate ${ }^{19,27,28}$. The contribution of active particles to the microstructure is less understood. They are expected to behave like a disordered fluid that surrounds the CNP network. This lack of order is inferred from the insignificance of both attractive and long-ranged repulsive forces; the former since otherwise the viscosity would be very high, the latter from the strong screening by the dissolved salt ${ }^{29}$.

These differences between SSFBs and conventional lithium-ion batteries raise the question, how degradation processes such as volume and structural changes of the active materials upon (de-) lithiation $^{11}$ or the formation of solid electrolyte interface (SEI) ${ }^{12,26}$ affect SSFBs. Several consequences of such processes for fluid electrodes are conceivable. Considering the CNP network, both the nature of the interparticle contacts and their number density can change: the former as a consequence of surface processes, and the latter due to (for flow batteries inherent) mechanical rejuvenation: shear-induced fragmentation of the CNP network creates a possibility for the fragments to re-assemble into a different microstructure 


\subsection{INTRODUCTION}

when the fluid returns to the quiescent state ${ }^{19}$. Macroscopically, the electronic conductivity and the yield stress are likely affected by these microscopic processes. While critically relevant to SSFBs, the above phenomena can potentially also affect other types of battery systems that use self-assembling colloidal particles, such as polysulphide ${ }^{10}$ and carbon free ${ }^{23}$ flow batteries. Recent work on carbon slurry based iron redox flow batteries has shown electronic conductivity enhancement through changes in the interparticle contacts due to iron plating ${ }^{22}$.

The objective of the present work is twofold: to quantify changes in rheological and electrical performance due to repeated electrochemical cycling, and to gain a mechanistic understanding of these macroscopic changes. To achieve these goals, a commercial rheometer was extended to allow parallel electrical impedance measurements, as well as a controlled cycling of the fluid electrodes via the inclusion of a lithium counter electrode. The studied fluid electrode consists of a mixture of Ketjen Black (KB) and $\mathrm{Li}_{4} \mathrm{Ti}_{5} \mathrm{O}_{12}$ (LTO) particles dispersed in EC:DMC 1:1 with $1 \mathrm{M} \mathrm{LiPF}_{6}$. LTO has previously been identified as a promising active material for $\mathrm{SSFBs}^{21}$ as lithiation occurs at about $\sim 1.55 \mathrm{~V}$ vs $\mathrm{Li} / \mathrm{Li}^{+}$, within the safe operating range of the non-aqueous electrolyte ${ }^{13,16,25}$. To examine the role of electrochemical state (and history), we cycle the fluid electrode to a series of increasingly reducing voltages, measuring the rheological and electrical properties before and after each charge and discharge step. Comparisons are also made before and after mechanical rejuvenation, to probe the changes in self-assembly. To facilitate interpretation of the various changes we also make a comparison between the results for the KB-LTO electrode, and a fluid electrode without the LTO. 


\subsection{Materials and Methods}

\subsubsection{Fluid Electrode Preparation}

Ethylene carbonate (EC) and dimethyl carbonate (DMC) were obtained from Sigma Aldrich (anhydrous, 99\%+ purity). Binary mixtures of EC and DMC were 1:1 by mass. LP30 (EC:DMC 1:1 with $1 \mathrm{M} \mathrm{LiPF}_{6}$ ) was obtained from BASF. Ketjen Black EC 600JD powder (KB) was obtained from AkzoNobel. $\mathrm{Li}_{4} \mathrm{Ti}_{5} \mathrm{O}_{12}$ powder was obtained from Südchemie. Lithium foil was purchased from Alfa Aesar (99.9\%). All sample preparations and experiments were carried out in an MBraun Argon-filled glove box $\left(\mathrm{O}_{2}, \mathrm{H}_{2} \mathrm{O}\right.$ below $\left.1 \mathrm{ppm}\right)$. Two fluid electrodes were prepared: a mixture of $1 \mathrm{wt} . \% \mathrm{~KB}$ and $5 \mathrm{wt} . \% \mathrm{LTO}$, and a reference sample at 1 wt.\% KB. The dry particles were first wetted by EC-DMC solvent for 8 hours to improve their dispersibility; from an earlier study it is known that KB is colloidally unstable in carbonate solvent with large amounts of salt ${ }^{29}$. The $\mathrm{KB}$ reference contained $2.9 \mathrm{wt} . \% \mathrm{~KB}$ while for the mixture it was $2.6 \mathrm{wt} . \% \mathrm{~KB}$ and 13.4wt.\% LTO at this stage. Afterwards, $\mathrm{LiPF}_{6}$ salt (Alfa Aesar (98\%)) was added via a concentrated solution $\left(\mathrm{LP} 30+\mathrm{LiPF}_{6}\right)$ to reach a concentration of $1 \mathrm{M}$ (viscosity $4 \mathrm{mPas}^{8}$ ). After an additional $8 \mathrm{~h}$, the samples were homogenized by rotor stator mixing (Ultraturrax) at $15000 \mathrm{rpm}$ for $2 \mathrm{~min}$, and loaded in the rheo-impedance setup.

\subsubsection{Cycling-rheo-impedance setup}

Electrochemical cycling and rheo-impedance measurements were performed on a stress controlled rheo-meter (Haake RS 600) with a home-built adaptation (Fig. 5.1) comprising an extension of a previously described system ${ }^{19}$. Briefly, the 


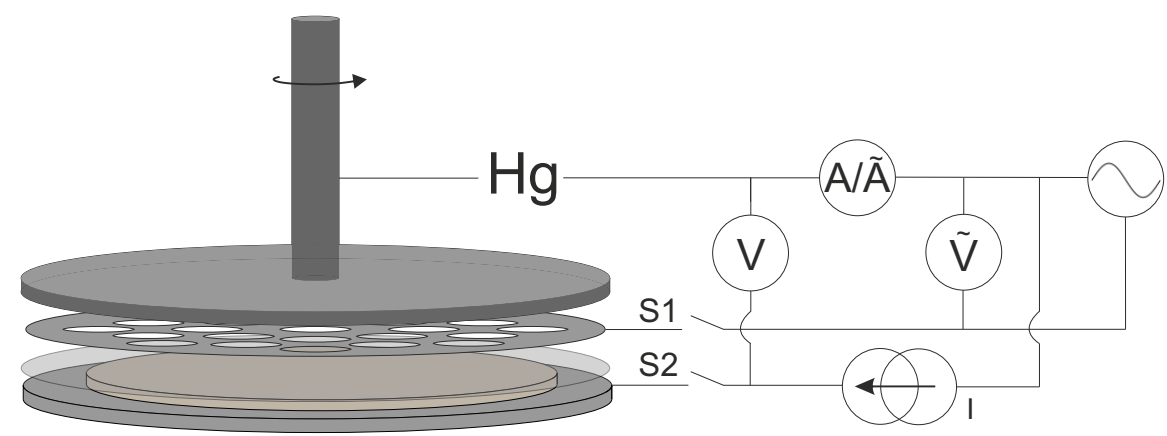

Figure 5.1: Schematic of rheo-impedance setup. dark grey: upper geometry, perforated plate, bottom current collector translucent gray: membrane, beige: lithium foil on current collector. Either switch S1 or S2 is closed, to allow EIS or electrochemical cycling.

$60 \mathrm{~mm}$ parallel plate geometry of the rheometer was used as a base. A copper plate attached to the upper rheometer rotor served as a shearing surface, current collector and electrode for electrical impedance spectroscopy (EIS). A perforated stainless steel (316) plate was used as the bottom shearing surface. Due to the small size $(1 \mathrm{~mm}$ radius) and fraction $(<40 \%)$ of the holes, rheological measurements could be performed with reasonable accuracy (within 5\%; test with 1.231 Pas and 0.01 Pas calibration oils). This plate also served as a second electrode for EIS. A Celgard 2500 separator was used to separate the perforated plate from a second compartment with a lithium foil (on a titanium current collector). This allowed the foil to be in contact the electrolyte but not the particles. In the solvent trap of the rheometer (not shown), mercury was used as a working fluid to ensure a low friction and low noise electrical connection to the rotating upper geometry ${ }^{19}$. During rheological tests all electrodes were disconnected. 
EIS measurements were performed (between the upper and perforated plate, with S1 closed and S2 open) in a four-terminal configuration. The perforated plate was excited by a sinusoidal voltage of $<50 \mathrm{mV}$ in the frequency range from $10 \mathrm{MHz}$ to $0.01 \mathrm{~Hz}$. Amplitude sweeps on the KB-only fluid had indicated that the response was linear (and hence the impedances the same) at least up to $100 \mathrm{mV}$ (Note: these voltages were applied at the HF2 output. The actual voltage across the sample was typically much smaller). Currents were measured by a transimpedance amplifier (HF2CA, Zurich Instruments) on the virtually grounded rotor. A buffer pre-amplifier (HF2TA, Zurich Instruments) was used to measure the potential difference between the perforated plate and the rotor. An impedance spectroscope (HF2IS, Zurich instruments) was used to extract the complex impedance from the current and voltage signals. The lithium electrode was allowed to float (it was disconnected from the external circuit) during these measurements. Frequency dependent parasitic impedances of the setup were calibrated out using the "open short" technique ${ }^{2,4}$.

Samples were electrochemically cycled (with S2 closed) using the lithium foil $\left(>30 \mathrm{~cm}^{2}\right)$ as a counter electrode and the Celgard membrane as an ion permeable medium. As the maximum current was low $\left(<50 \mu \mathrm{A} \mathrm{cm}^{-2}\right)$ the total polarization was below $50 \mathrm{mV}^{3,15}$. Currents were measured through a $50 \Omega$ resistor. The potential of the perforated plate was allowed to float during cycling. Galvanostatic and potentiostatic charging were performed using the impedance spectroscope. Custom LabVIEW codes were used to perform cycling and EIS and to synchronize them with rheological measurements. 


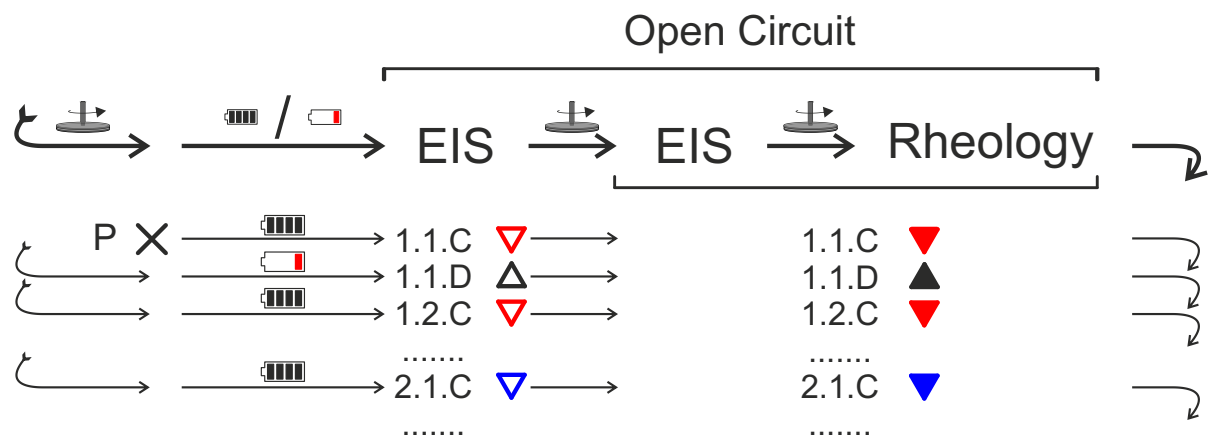

Figure 5.2: Schematic of the (repetitive) measurement protocol. Pictograms indicate mechanical rejuvenation and electro-chemical (dis)charging. The rejuvenation serves to create a reference state by breaking down the particle agglomerates and letting them re-assemble again. Since EIS does not involve mechanical deformation, measurements before and after rejuvenation can be compared.

\subsubsection{Experimental Protocol}

All surfaces in contact with the sample (excluding the lithium foil and the separator) were sand papered and thoroughly cleaned outside the glovebox prior to the experiment. They were subsequently wetted with DMC for 15 min prior to sample loading in the glovebox. Cycling-rheo-impedance experiments were performed with a gap of $250 \mu \mathrm{m}$ between upper current collector and perforated plate. To avoid sample variations due to differences in shear history ${ }^{19}$, we presheared each sample at $1000 \mathrm{~s}^{-1}$ for $200 \mathrm{~s}$, and subsequently allowed them 200 s of rest. This 'mechanical rejuvenation' was applied before each cycling (charging or discharging) step. Rheo-impedance measurements were carried out both before and after this treatment. The lithium electrode was disconnected during rheo-impedance measurements. A scheme of the protocol is given in Fig. 5.2.

To indicate the electrochemical history, we code our samples as follows: (Volt- 
age window number). (Cycle number). (Charge (lithiation)/ Discharge (delithiation) step). The pristine state is denoted as P. For example, code 2.3.C represents the state reached in the 2 nd voltage window after charging the fluid for the $3 \mathrm{rd}$ time under these conditions. Moreover, each sample has two mechanical states: before or after mechanical rejuvenation.

Samples were (dis-) charged galvanostatically using a current of $1.5 \mathrm{~mA}$. Assuming an LTO concentration of $(5 \mathrm{wt} . \%=) 0.121 \mathrm{~g} \mathrm{ml}^{-1}$ and a specific capacity of $175 \mathrm{mAh} \mathrm{g}^{-1}$, this corresponds to a rate of about $\mathrm{C} / 10$. Once the cutoff voltage was reached, the voltage was held until the current fell below $0.5 \mathrm{~mA}$. Thereafter EIS measurements were performed, taking into account the aforementioned mechanical protocol. Next the yield stress was measured by ramping up the shear stress (62 s per stress decade) while measuring the strain. The log(strain) versus $\log ($ stress $)$ curve was fitted with two straight lines and the stress at the intersection was taken to be the yield stress ${ }^{24}$. The flow curve was subsequently measured by pre-shearing at $1000 \mathrm{~s}^{-1}$ for $200 \mathrm{~s}$ and then slowly stepping the shear rate downwards from $1000 \mathrm{~s}^{-1}$ (at 20 steps per shear rate decade). After an equilibration time of $20 \mathrm{~s}$ the viscosity was averaged over one second. Six charge and discharge half-cycles were performed for each voltage window. At the end of the experiment, samples were recovered, dried at $60 \mathrm{C}$ and then analyzed post mortem outside the glovebox. 


\subsection{RESULTS AND DISCUSSION}

\subsection{Results and Discussion}

SSFB fluid electrodes conduct through ionic and electronic pathways ${ }^{9,27}$. As the metal current collectors that enclose the fluid electrode are ionically blocking but electronically reversible, these two contributions can (in principle) be separated using impedance spectroscopy ${ }^{14,19}$. The electronic resistance of the fluid electrode's percolated particle network (with some contributions from the current collector interface ${ }^{19,27}$ ) corresponds to the low-frequency limit of the real impedance. Experimental timescales do not always allow access to this limit, and therefore the low frequency real impedance (LFRI see Fig. 5.3 panel B) at $0.01 \mathrm{~Hz}$ was taken as practical measure (suitable for identifying trends) of the electronic resistance. Further justification of this approach will be presented in Fig. 5.4B, where we also fit LFRI values using an equivalent electrical circuit model.

Figure 5.3 shows the impedance, yield stress and viscosity (from now on termed together as rheo-impedance) of a fluid electrode containing 1wt.\% KB and 5wt.\% LTO, cycled between 1.4-2.5 V and 1.0-2.5 V. Most measurements (solid symbols) were performed after mechanical rejuvenation of the fluid; we will focus on these first.

In regime $\mathrm{I}$, with a less reducing cut-off of $1.4 \mathrm{~V}$, both the LFRI and the yield stress show an alternating behavior on cycling, with a higher LFRI and a lower yield stress at $1.4 \mathrm{~V}$ as compared to $2.5 \mathrm{~V}$. The difference in the LFRI at the two states of charge becomes progressively smaller upon cycling, while the difference in yield stress remains roughly the same. Subsequent cycling in regime II, with a cutoff of $1.0 \mathrm{~V}$, leads to strong changes. The LFRI triples, and further alternation is suppressed. The yield stress roughly halves, but here the 

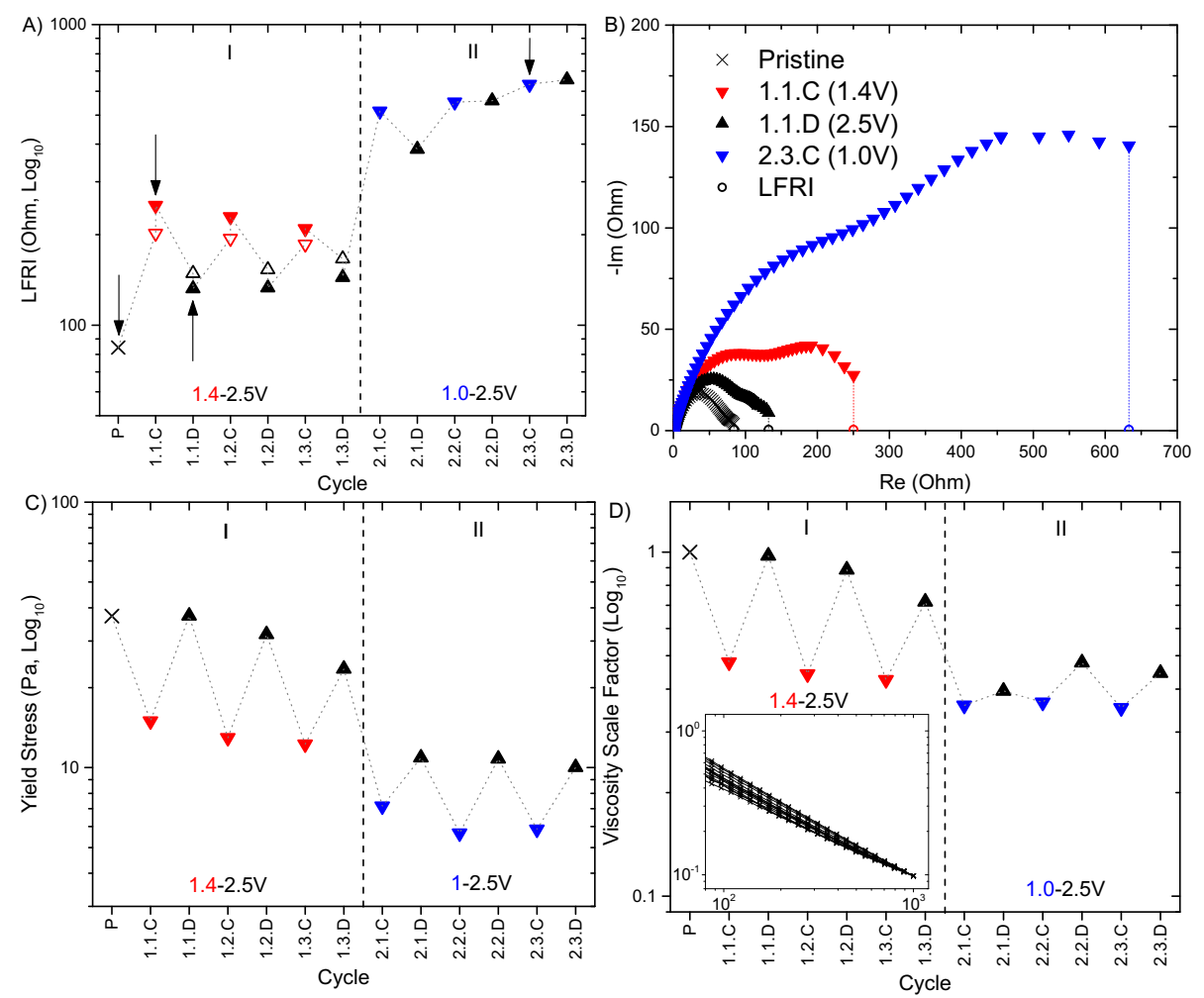

Figure 5.3: Rheo-impedance of 1wt.\% KB+5wt.\% LTO fluid electrode subject to cycling in different voltage ranges. $(X)$ : pristine state, $(\Delta)$ : discharged to $2.5 \mathrm{~V}$, $(\nabla)$ : charged to indicated voltage. Open symbols: not mechanically rejuvenated, Closed symbols: mechanically rejuvenated. A) Low frequency real impedance from EIS spectra obtained at the end of charge or discharge. B) Nyquist plots of data points marked with arrows in A. The dotted lines connect the lowest frequency impedance to the LFRI C) Yield stress. D) Viscosity scale factor: the factor with which the viscosity at $1000 \mathrm{~s}^{-1}$ has to be multiplied to coincide with that of the pristine state. The inset shows the flow curves (viscosity in Pas vs. shear rate in $\left.s^{-1}\right)$ after multiplication with the VSFs. 


\subsection{RESULTS AND DISCUSSION}

alternations remain (Fig. 5.3C). The loss of alternations in the measured LFRI may be due to experimental limitations: as shown by the Nyquist plot (Fig. $5.3 \mathrm{~B})$, the time constant of the low frequency arc shows a large increase, thereby compromising the sensitivity of the LFRI to the electronic resistance.

Changes in the rheological properties are reflected in not just the yield stress but also the flow curves. Inspection of the latter reveals that the shear-rate dependence of the viscosity is rather similar for all samples (inset Fig. 5.3D). This allows representation of the effects of electrochemical cycling via a viscosity scale-factor VSF (main panel of Fig. 5.3D). The yield stress and VSF show a similar dependence on the electrochemical state; lithiation lowers both quantities and vice versa for delithiation. Changing to regime II, i.e. the cycling between 2.5 V-1.0 V, the yield stress and viscosity reduce. Again these changes correlate well with the higher LFRI, with the exception that alternations remain in the yield stress and VSF. This may be due to the aforementioned issue with the LFRI.

Interpretation of the progressive changes in rheo-impedance of the fluid after cycling is not trivial. One aspect hereof is that the duration of the charge and discharge was not the same for each cycle (see SI Fig. 5.8, and Table 5.1). For this reason, only relative changes caused by electrochemical cycling (and mechanical rejuvenation) will be discussed. Furthermore, the measurements are performed in a complex system, consisting of several instrumental parts and a multicomponent fluid electrode. Analyzing the electrical and rheological data in conjunction, the scope of interpretation can however be narrowed down. Importantly, the electrical and mechanical signals originate from the same system, comprising a bulk fluid between the same two metal plates (the upper current collector and 
the perforated plate).

This still leaves the question, whether contributions from the metal-fluid interfaces can be neglected or not. A significant interfacial contribution to the rheological signal would require a mechanically weak layer near the rheometer geometry walls (e.g. due to depletion of particles or weak particle-wall interactions). There are however no indications for this. First, the yield stress curves (see SI Fig. 5.10) indicate an initial elastic deformation and finite strain at yield (also for samples with a very high LFRI). This corresponds well to a gap-spanning network, whereas a weak interfacial layer would already yield (i.e. flow) at an infinitesimal strain. Secondly, the viscosities (at high shear rates) change appreciably with each cycling step, implying that the forces responsible for particle agglomeration should do the same ${ }^{19}$. This is only possible if the particles themselves undergo changes. The absence of a particle-depleted layer at the metal plates, as inferred from the rheology, suggests that the LFRI signal is dominated by the fluid bulk. From a different perspective, since the gap $(250 \mu \mathrm{m})$ between the measuring surfaces for EIS spans $\mathrm{O}(1000)$ particle diameters, the number of particle contacts involved in an electron conduction path has to be very large, as compared to the single particle-metal contact per current collector. It is thus appropriate to seek an explanation of the observations in Figure 5.3 in terms of changes to the particles (and not the enclosing metal surfaces).

Since SSFB electrodes are multi-component mixtures, the effects of electrochemical cycling are not limited to just one component. However, additional observations help identify the most dominant changes. Examination of the LTO particles (both in pristine state, and after cycling) revealed that no structural decomposition could be detected with XRD (see SI Fig. 5.11). The higher elec- 


\subsection{RESULTS AND DISCUSSION}

tronic conductivity of carbon blacks compared to LTO suggests that they will have a dominant influence on the suspension electronic conductivity. Moreover, the different rheologies of suspensions of only KB (yield stress, higher viscosity) and only LTO (no yield stress, lower viscosity) in the same solvent (see SI Fig. 5.12) suggests that the carbon black particles have a dominant influence on the rheology. In absence of an all-encompassing model for how electronic conductivity and yield stress are generated in the fluid electrodes, it is thus very reasonable to assume that the dominant contribution to both signals comes from the KB.

To demonstrate this further, we consider a similar measurement on a sample that contains only KB as a particulate component (Fig. 5.4). This sample was cycled in four voltage ranges, where the first two correspond to those of the KB-LTO fluid electrode (see SI Fig. 5.9 and Table 5.1). In regime I (1.4 V cutoff), the behavior of the KB fluid electrode is similar to that of the KB-LTO fluid electrode. In the subsequent, more reducing regimes II and III (with cutoff voltages of 1.0 and $0.8 \mathrm{~V}$ respectively), the differences between the 'charged' (reduced) and 'discharged' states progressively become larger, spanning almost an order of magnitude for both the LFRI and yield stress. The alternation of both properties appears to be repeatable in regimes II and III. However, cycling in regime IV with a cutoff of $0.6 \mathrm{~V}$ (well outside the stability window of the electrolyte) results in a massive increase in the LFRI and decrease in the yield stress and VSF. No clear trends are observed in the LFRI or rheological properties for subsequent cycles.

While the voltage ranges appear slightly shifted, the behavior of the KB-only fluid electrode qualitatively agrees with that of the KB-LTO mixture. We can thus attribute the behavior of both systems to the KB network. Within this 

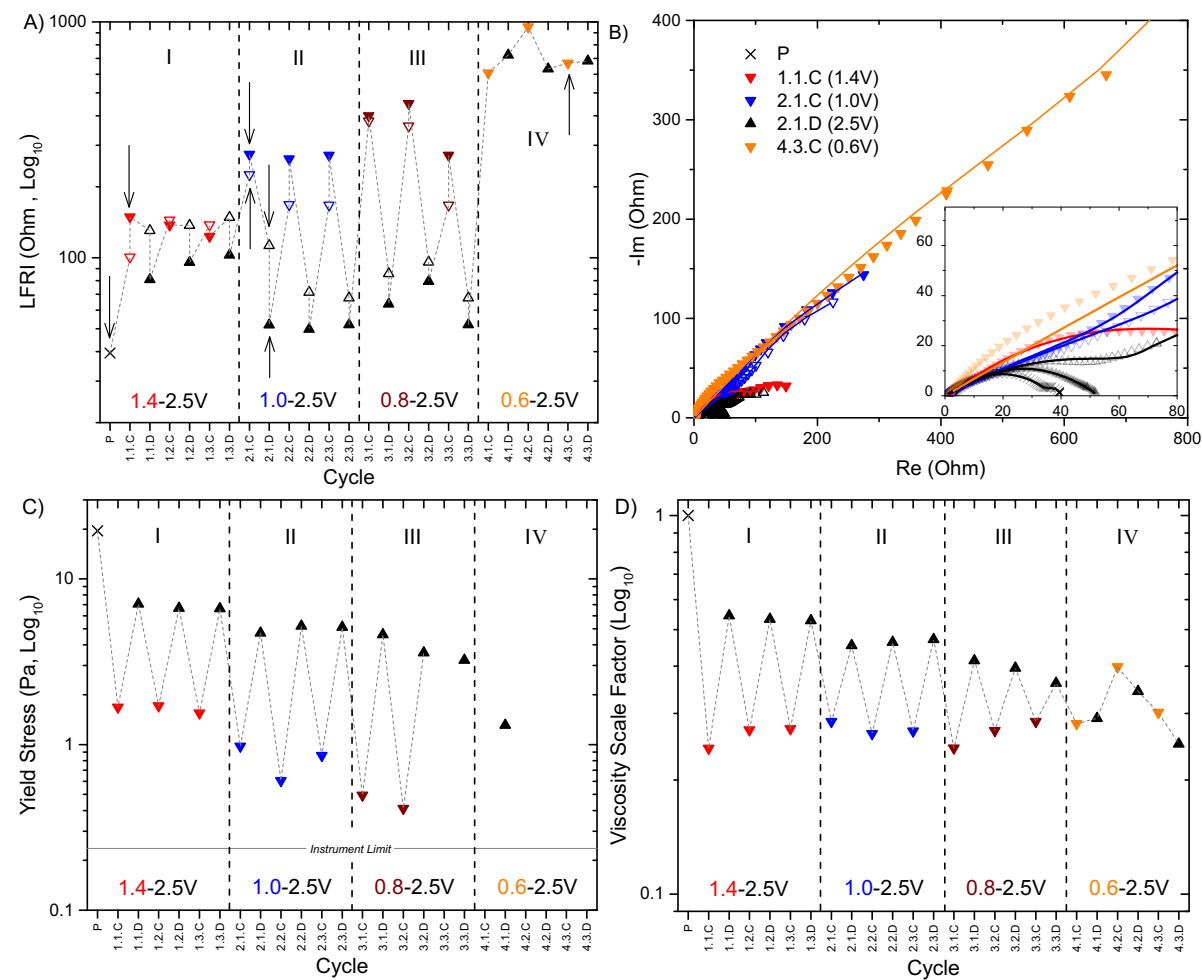

Figure 5.4: Rheo-impedance of 1 wt\% KB fluid electrode subject to cycling. Symbols are the same as Fig. 5.3. A) LFRI. B) Nyquist plots of data points marked with arrows in $A$ and fits (described later) using the equivalent circuit in fig. 5.6 C) Yield stress. D) Viscosity scale factor, defined similarly as in Fig. 5.3.

focused interpretation, the LFRI trends in Figs 5.3 and 5.4 can be rationalized by the formation of an insulating SEI layer around the KB particles upon exposure to reducing voltages, and a partial dissolution of this layer during delithiation (2.5 V). Recent studies have demonstrated that the composition and properties of the SEI depend on the potential versus lithium where it is formed ${ }^{1,7,17}$. At higher voltages, a sparse and less insulating SEI layer composed of organic compounds 


\subsection{RESULTS AND DISCUSSION}

(more prone to dissolution) is formed. At lower potentials; a thicker, denser, less soluble, and more insulating layer composed of inorganic compounds gets formed.

Consistency with the observed changes in the rheological properties implies that the layer must also weaken the attractive forces that hold the KB network together. In the electrostatically screening environment of the $1 \mathrm{M}$ salt solution this is possible through reduced van der Waals attractions. Assuming that the (typical) contact geometry between two sticking KB units remains the same, this would suggest a lowered Hamaker constant.

It may seem surprising that we observe strong effects of a SEI layer within the 'safe' operating range of the electrolyte. This may be due to an uneven current distribution caused by the inhomogeneity of fluid electrodes. This could lead to variations in local particle states of charge, triggering reductive electrolyte decomposition and SEI formation ${ }^{6,18,20}$.

On cycling the fluid electrode to lower voltages, a thicker SEI forms, with a drastic effect on the electronic resistance. It should be noted that the increase in the electronic resistance due to charging (lithiation), is larger than indicated by the LFRI. This is easily recognized from Figures $5.3 \mathrm{~B}$ and $5.4 \mathrm{~B}$, in which the low-frequency semi-circle is far from complete at $0.01 \mathrm{~Hz}$, in particular for the lithiated state. The large difference (more than one order of magnitude) in the LFRI between $1.0 \mathrm{~V}-2.5 \mathrm{~V}$ and $0.8 \mathrm{~V}-2.5 \mathrm{~V}$ of the fluid electrode only containing $\mathrm{KB}$ indicates that the difference in electronic conductance must be huge, even for voltages $\geq 1.0 \mathrm{~V}$ vs the Li counter electrode. At lower (more reducing) voltages, the effect of the irreversible process on the electronic resistance is probably even larger.

To further examine the formation of surface layers, we compare the LFRI be- 


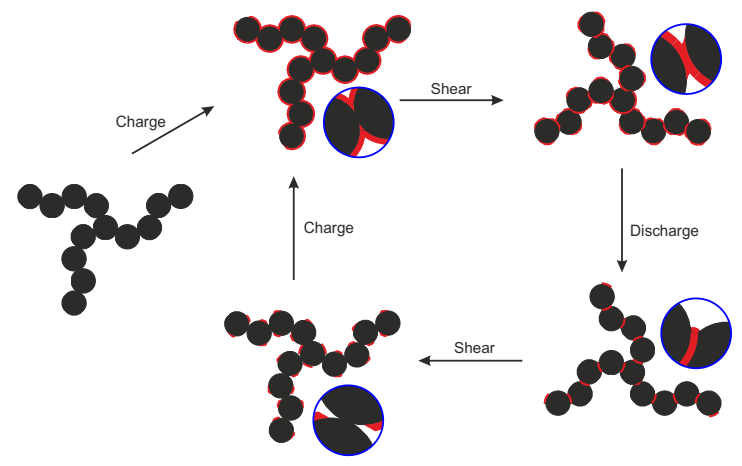

Figure 5.5: Proposed mechanism to explain the observed combined effect of (dis)charging to higher potentials and subsequent mechanical rejuvenation on the LFRI (i.e. the conductivity of the KB network). Red color indicates (in an exaggerated way) the presence of an SEI layer, which grows during charging, and shrinks during discharging. Particle contacts are less strongly affected by layer deposition or dissolution. Note: in reality the primary KB particles are fractallike, and re-assembly after shear leads to a different (but statistically equivalent) network.

fore (open symbols) and after (closed symbols) mechanical rejuvenation in Fig. 5.3 and 5.4. For both samples it is clear that restructuration by shear (followed by rest), consistently increases the LFRI after charging and decreases it after discharging. Assuming that the pre-shear breaks down all agglomerates, and that the re-agglomeration process is not impeded by energy barriers (diffusion limited agglomeration ${ }^{5}$ ), the micro-structure after rejuvenation will (statistically) be the same. However, the conductivity of interparticle contacts will have changed. This is because the contacts between single particles of the network are less likely to be affected by the formation or dissolution of SEI, as they are less exposed to the electrolyte solution. Consequently, less SEI is formed at the contacts during charging (lithiation) and likewise less is dissolved during discharging (delithia- 


\subsection{RESULTS AND DISCUSSION}

tion). Mechanical rejuvenation leads to randomization of the contacts. Thus, the subsequently formed contacts will contain mainly maximally grown (or maximally dissolved) SEI. This mechanistic explanation, illustrated in Figure 5.5, thus supports that the formation of the insulating SEI is partially reversible at higher potentials, i.e. lower state of charge. Ellipsometry measurements (see SI Fig. 5.13) of SEI growth and dissolution on a carbon thin film corroborate this. We remark here that the observed reversibility very likely depends on the duration that the fluid electrode potential is held outside of the electrochemical stability window of the electrolyte. Also, as the reversibility of the SEI (and its composition) depends on the potential at which it is formed, this mechanism is no longer valid at very low potentials $(0.6 \mathrm{~V})$ where permanent SEI is formed.

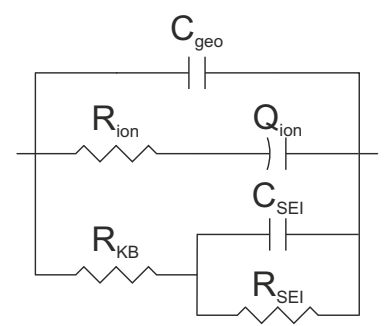

Figure 5.6: Equivalent circuit used to fit the data of Fig. 5.4 A.

The identification of SEI as the cause of impedance changes makes it interesting to extract the electronic resistances by modelling the impedance spectra of the KB only system using a simplified equivalent circuit (Fig. 5.6). The ionic contribution can be modeled as an ionic resistance $R_{\text {ion }}$ in series to a constant phase element $Q_{i o n}$ that represents the double layer capacitances of the electrolyte interfaces. To model the electronic part, we use a resistor RKB that represents the summed KB intra-particle resistances in series with a parallel resistor RSEI and 
capacitor CSEI that accounts for the summed interparticle impedances (due to SEI). A capacitor $C_{g e o}$ in parallel to the rest of the circuit represents the geometric capacitance of the system. The fits (Fig. 5.4 B inset, additional fits in SI) show good agreement with the spectra.

We also note that the measured LFRI and the fitted electronic resistance (the sum of the KB inter and intra particle resistances) in Fig. 5.7 A show the same trends, thereby justifying our earlier given interpretation of the changes in LFRI. Clearly, and as expected, the measured LFRI values under predict the electronic resistance for most lithiated states. In Fig. $5.7 \mathrm{~B}$ we decompose the fitted LFRI values into the contributions $R_{K B}$ and $R_{S E I}$. This comparison shows that the most important changes in the total electronic resistance comes from the interparticle resistance.
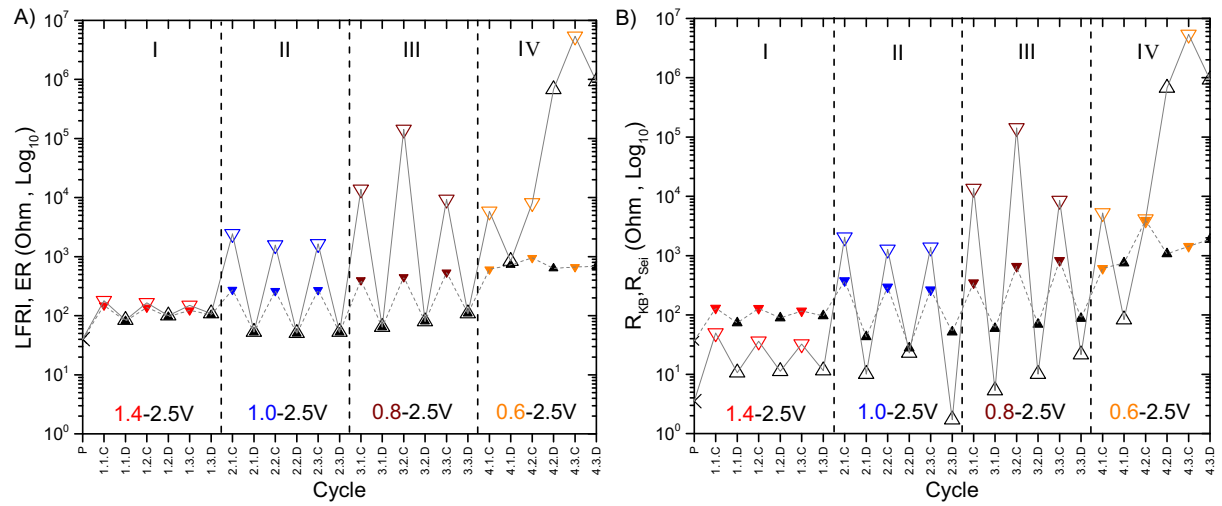

Figure 5.7: Results of the fits of spectra of the mechanically rejuvenated samples in Fig. 5.4A. A) Comparison of total electronic resistance and LFRI. B) Comparison of fit summed $K B$ interparticle $\left(R_{S E I}\right)$ and intraparticle $\left(R_{K B}\right)$ electronic resistances. 


\subsection{Conclusions}

Our study of the effect of cycling on the rheo-impedance of a LTO-based SSFB anolyte has produced several new insights. Two key properties are very sensitive to the electrochemical state and history: lithiation causes the electronic resistance (LFRI) to increase and the yield stress to decrease, and vice versa for delithiation. A lithiation voltage below $1.0 \mathrm{~V}$ vs a Li electrode causes a drastic increase in LFRI. A suspension of only KB particles responds in a similar way to electrochemical state and history, indicating that the observed effects of cycling can be largely attributed to the KB. A simple mechanistic picture that captures most findings, is one in which the KB particles get covered by a SEI layer during charge (lithiation). For less reducing, i.e. higher voltages, a part of this layer dissolves during discharge. The layer electrically insulates the KB particles and diminishes their attractions. The contact points between the KB units are less affected by the layer growth, similar to conventional solid lithium batteries. However, in SSFBs, a mechanical rejuvenation of the structure takes place every time the fluid gets pumped, leading to the incorporation of the thicker layers into the KB backbone. For strongly reducing voltages a permanent SEI layer is formed.

The implications of our findings for SSFBs are significant. A low electronic resistance is crucial to battery performance, and a sufficient yield stress is required to suspend active materials. Both these properties are adversely affected by SEI formation under the explored experimental conditions. Our findings show that further research into chemistries with absolutely no (insulating) layer formation would be required to realize SSFBs. 


\subsection{Supplementary Information}

Discussion of Table 5.1: The contribution of LTO to the capacity can potentially be estimated by subtracting the contribution of the KB-only system from that of the KBLTO fluid electrode. It is clear that for the initial cycles of the 2.5-1.0 $\mathrm{V}$ window (2.1.C and 2.1.D) the KB-LTO mixture has a much higher capacity. The difference in capacity is close to the LTO's theoretical capacity of $7.8 \mathrm{mAh}$. This along with the voltage plateau in the KB-LTO charge curves (absent in the KB-only ones) indicates that a significant fraction of the LTO participates during the initial cycle at $1.0 \mathrm{~V}$. In the previous and subsequent cycles, the capacities of $\mathrm{KB}$ and $\mathrm{KB}-\mathrm{LTO}$ are similar suggesting that most of the charging current is used to form (partially reversible) SEI.

While the above trends are clear, caution is required while making quantitative comparisons of the capacities of the two fluid electrodes. They exhibit different impedances and thus overpotentials while charging. This could explain why the KB only fluid electrode (with a lower initial LFRI) has a higher capacity than the mixture for the first cycle of the 2.5-1.4 V window. Other complicating factors that cannot be generally excluded are small leakage currents, and self-discharge during rheo-impedance tests. While these uncertainties make a quantitative analysis of the amount of (dis)charge difficult, they should have no implications for our interpretation of cycling induced changes in the rheology and LFRI. 


\begin{tabular}{|c|c|c|c|c|c|c|c|c|c|c|c|c|c|c|c|c|c|c|}
\hline & \multicolumn{3}{|c|}{ x.1.C } & \multicolumn{3}{|c|}{ x.1.D } & \multicolumn{3}{|c|}{$\mathrm{x} .2 . \mathrm{C}$} & \multicolumn{3}{|c|}{$\mathrm{x} .2 . \mathrm{D}$} & \multicolumn{3}{|c|}{$\mathrm{x} .3 . \mathrm{C}$} & \multicolumn{3}{|c|}{ x.3.D } \\
\hline & Galv. & Pot. & Tot. & Galv & Pot. & Tot. & Galv. & Pot. & Tot. & Galv & Pot. & Tot. & Galv. & Pot. & Tot. & Galv. & Pot. & Tot. \\
\hline & & & & & & & & & $2.5-1$ & $.4 \mathrm{~V}$ & & & & & & & & \\
\hline KBLTO & 1.5 & 0.9 & 2.4 & 0.7 & 0.5 & 1.2 & 0.6 & 0.9 & 1.5 & 1.0 & 0.5 & 1.5 & 0.6 & 1.3 & 1.9 & 1.4 & 0.6 & 2.0 \\
\hline $\mathrm{KB}$ & 2.4 & 1.3 & 3.7 & 0.7 & 0.4 & 1.1 & 0.7 & 0.5 & 1.2 & 0.6 & 0.3 & 0.9 & 0.7 & 0.4 & 1.1 & 0.6 & 0.3 & 0.9 \\
\hline KBLTO- & & & -1.2 & & & 0.1 & & & 0.3 & & & 0.5 & & & 0.8 & & & 1.1 \\
\hline & & & & & & & & & $2.5-$ & $1 \mathrm{~V}$ & & & & & & & & \\
\hline KBLTO & 5.6 & 6.6 & 12.2 & 0.2 & 12.1 & 12.3 & 0.5 & 2.4 & 2.9 & 0.4 & 3.2 & 3.6 & 0.4 & 2.5 & 2.9 & 0.2 & 3.0 & 3.2 \\
\hline $\mathrm{KB}$ & 2.9 & 2.2 & 5.0 & 1.9 & 1.1 & 3.0 & 0.7 & 1.9 & 2.6 & 1.4 & 0.6 & 2.0 & 0.8 & 1.7 & 2.5 & 1.3 & 0.6 & 1.9 \\
\hline $\begin{array}{l}\text { KBLTO- } \\
\text { KB }\end{array}$ & & & 7.2 & & & 9.3 & & & 0.3 & & & 1.6 & & & 0.4 & & & 1.4 \\
\hline & & & & & & & & & $2.5-0$ & $.8 \mathrm{~V}$ & & & & & & & & \\
\hline KB & 1.4 & 2.4 & 3.9 & 0.7 & 1.4 & 2.0 & 3.1 & 2.3 & $\begin{array}{l}\mathbf{5 . 4} \\
2.5-0\end{array}$ & $\begin{array}{r}0.7 \\
.6 \mathrm{~V}\end{array}$ & 1.2 & 1.9 & 5.4 & 3.7 & 9.2 & 1.0 & 0.9 & 1.9 \\
\hline KB & 1.9 & 0.9 & 2.8 & 0.8 & 0.1 & 0.9 & 0.1 & 13.6 & 13.6 & 0.1 & 0.0 & 0.1 & 0.1 & 0.0 & 0.1 & 0.1 & 0.0 & 0.1 \\
\hline $\begin{array}{l}\text { Table } 5.1 \\
\text { theoretical }\end{array}$ & har & 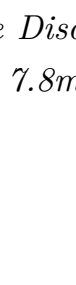 & $A h$. & t & 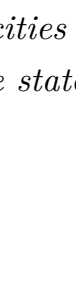 & 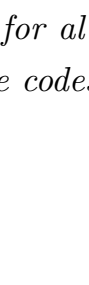 & & 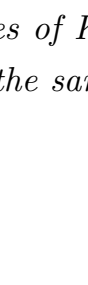 & $R I$ & 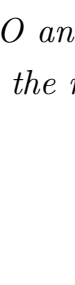 & . & . & $i d$ & & & $\begin{array}{l}\text { Units } \\
\text { oltag }\end{array}$ & Ah. & $\begin{array}{l}\text { LTO } \\
w) .\end{array}$ \\
\hline
\end{tabular}



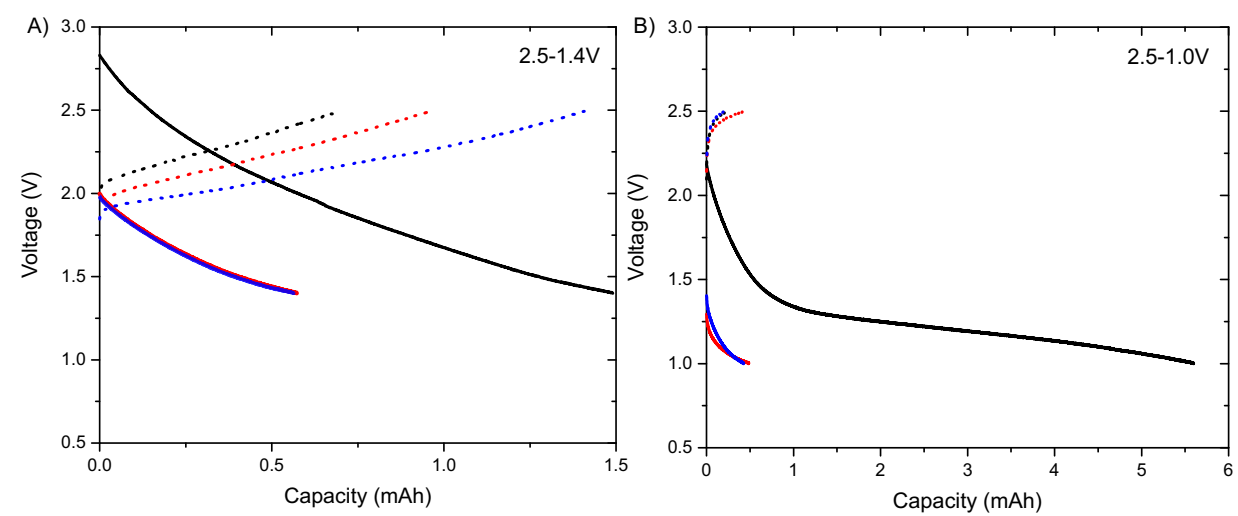

Figure 5.8: Galvanostatic charge and discharge profiles of the mixed KB (1 wt\%) + LTO (5 wt\%) suspension for two voltage windows (performed subsequently, in the order of lettering). Within each window, the first cycle is indicated black, the second in red and the third in blue. Charging is indicated by solid lines, discharging by dotted lines. 

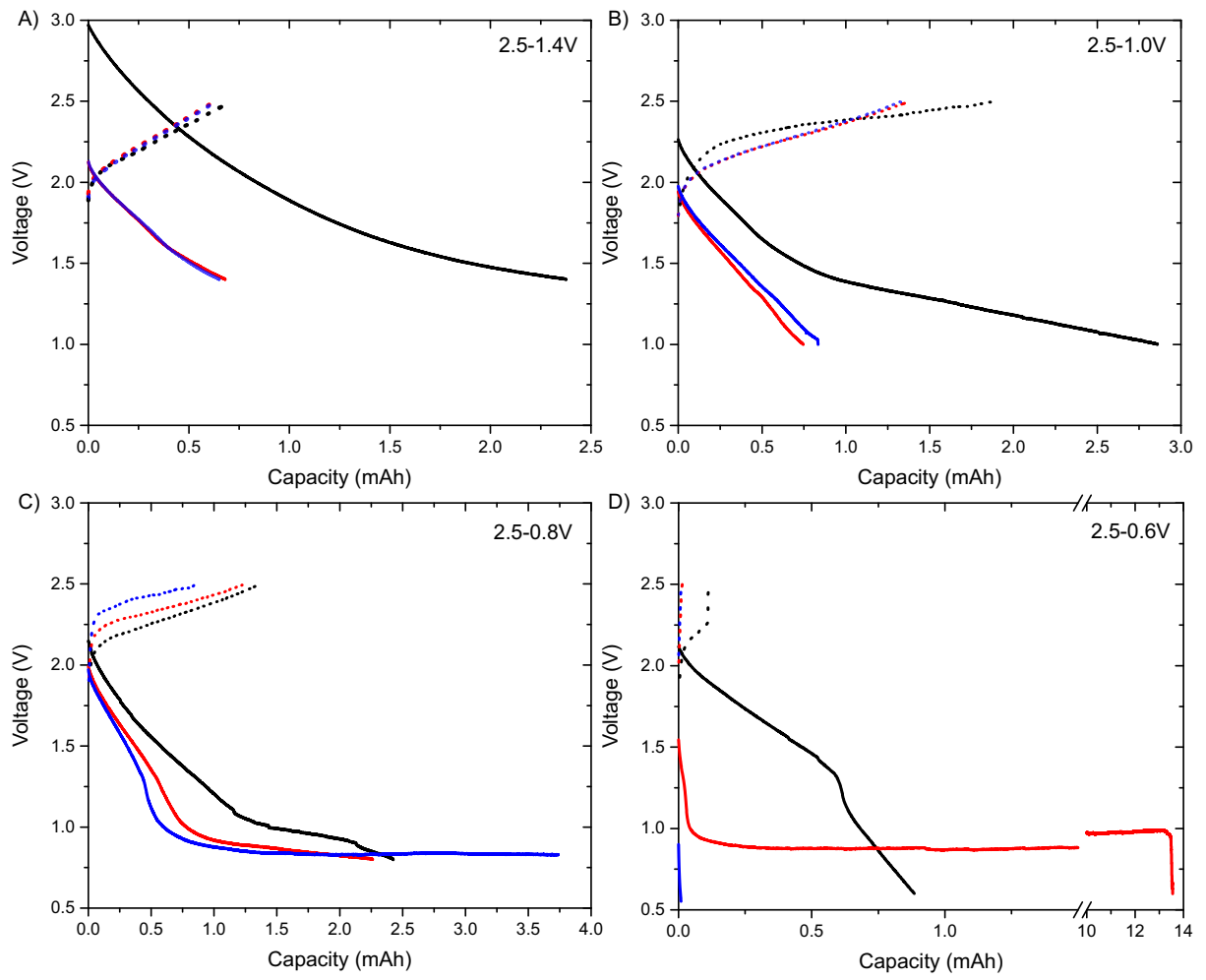

Figure 5.9: Galvanostatic charge and discharge profiles of KB suspension over four voltage windows (performed in the order of lettering). Within each window, the first cycle is indicated black, the second in red and the third in blue. Charging is indicated by solid lines, discharging by dotted lines. 


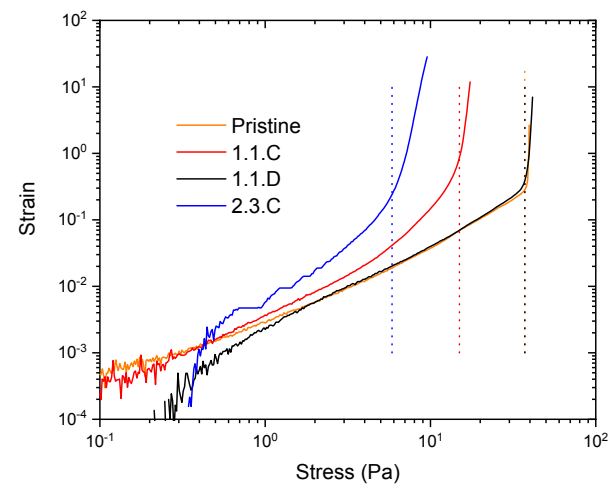

Figure 5.10: Measured strain versus applied stress of the mixed KB+LTO suspension, for the states indicated in Fig. 5.8. The dashed vertical lines indicate the extracted yield stress.

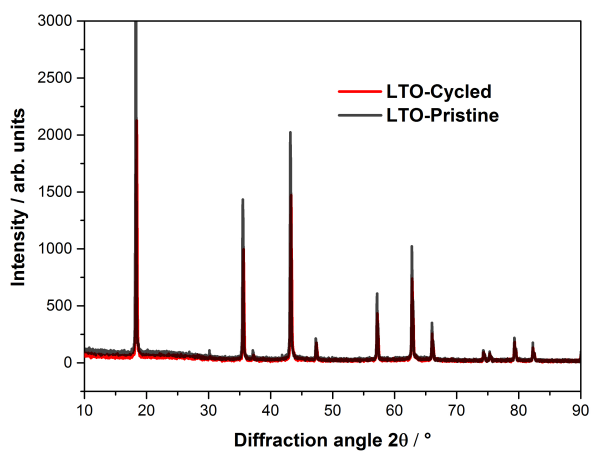

Figure 5.11: X-ray diffractograms (Bruker D8 Advance using CuK $\alpha$ radiation) of pristine and cycled (dried after cycle 2.3.D) active material. 


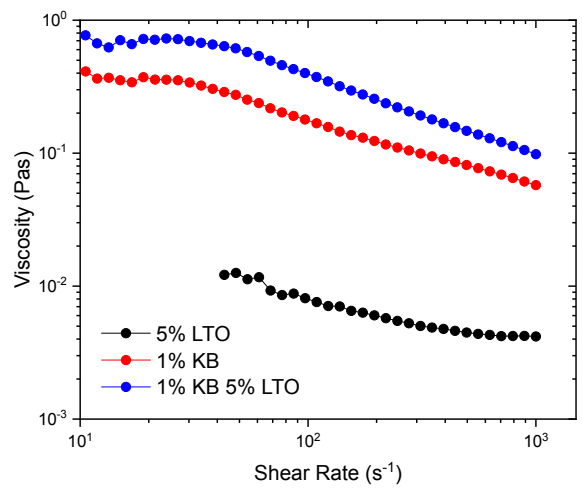

Figure 5.12: Viscosities versus shear rate (flow curves) of suspensions of $K B$, $L T O$ and $K B+L T O$ in EC:DC $+1 M L_{L P F}$. Concentrations are expressed in $w t . \%$.

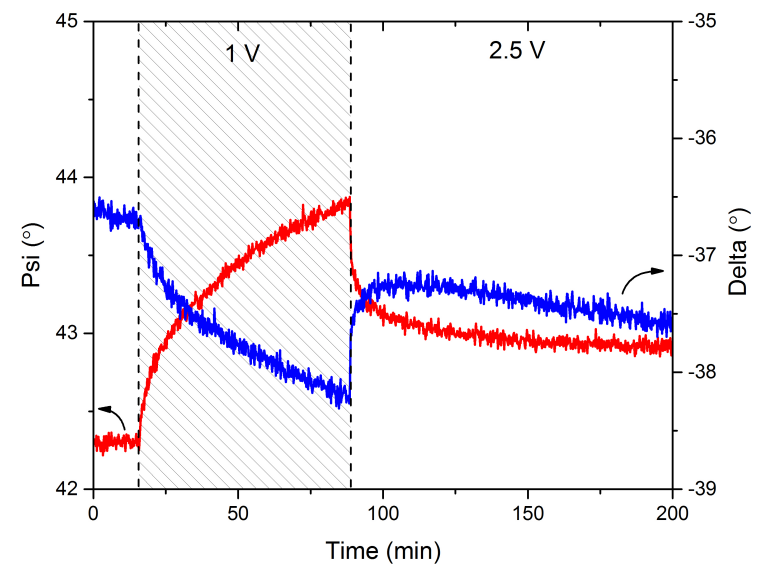

Figure 5.13: Time dependence of the ellipsometric angles of a carbon-coated multi-layer sample in EC:DMC 1:1 + $1 M \mathrm{LiPF}_{6}$, during subsequent exposure to 1.0 and $2.5 \mathrm{~V}$ versus a Li counter electrode. Psi angles are indicated in red, while Delta angles are shown in blue. 
Discussion of Fig. 5.13: The sample consisted of a thin layer $(\sim 81 \mathrm{~nm})$ of sputtered carbon on copper, immersed in EC:DMC 1:1 + 1M LiPF 6 . The counter electrode was a lithium foil. Data was collected on a Woollam M2000 eliipsometer (wavelength: $800 \mathrm{~nm}$ ). A Q-Sense 401 ellipsometry cell was modified to include an electrical connection to the sensor surface to allow in situ electrochemistry. Allowing the pristine sample to be at open circuit potential (absence of a current) results in almost constant Psi and Delta angles, suggesting the absence of either dissolution of layers or deposition of new material. Strong and ongoing changes in both ellipsometric angles are observed when the voltage is set to a $1.0 \mathrm{~V}$ ('charging'), while setting the voltage to $2.5 \mathrm{~V}$ ('discharging') results in a partial recovery of both Psi and Delta angles.

Since the copper and carbon do not dissolve under these conditions, the changes in the ellipsometric angles during exposure to $1.0 \mathrm{~V}$ must be due to the deposition of a new material on the substrate. This makes it likely that the partial reversal of these changes on exposure to $2.5 \mathrm{~V}$ are due to a partial dissolution of this layer.

We acknowledge Prof. Nieck Benes of the Films in Fluids group and Prof. Weibe de Vos of the membrane Science and Technology Group at the University of Twente for granting access to the ellipsometer. 


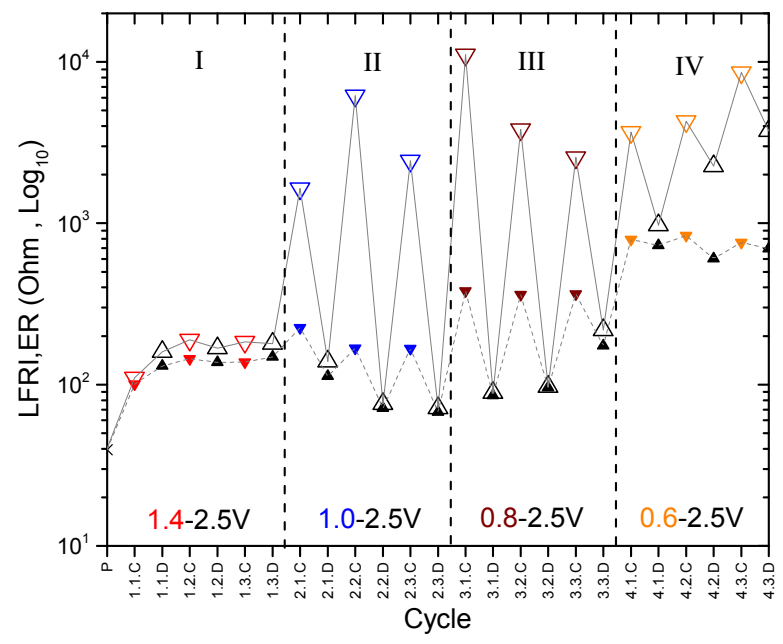

Figure 5.14: Comparison of the LFRI (closed symbols) and the fit Electronic Resistance (ER, open symbols) for the not mechanically rejuvinated states for the $K B$ only sample. The equivalent circuit used to obtain ER is indicated in Fig. 5.7 of the main manuscript. 


\section{Bibliography}

[1] S. J. An, J. Li, C. Daniel, D. Mohanty, S. Nagpure, and D. L. Wood. The state of understanding of the lithium-ion-battery graphite solid electrolyte interphase (sei) and its relationship to formation cycling. Carbon, 105:52-76, 2016.

[2] E. Barsoukov and J. R. Macdonald. Impedance spectroscopy: theory, experiment, and applications. John Wiley \& Sons, 2005.

[3] B. Burrows and R. Jasinski. The li/li+ reference electrode in propylene carbonate. Journal of the Electrochemical Society, 115(4):365-367, 1968.

[4] L. Callegaro. Electrical impedance: principles, measurement, and applications. CRC Press, 2012.

[5] M. Cerbelaud, B. Lestriez, R. Ferrando, A. Videcoq, M. Richard-Plouet, M. T. Caldes, and D. Guyomard. Numerical and experimental study of suspensions containing carbon blacks used as conductive additives in composite electrodes for lithium batteries. Langmuir, 30(10):2660-2669, 2014.

[6] W. C. Chueh, F. El Gabaly, J. D. Sugar, N. C. Bartelt, A. H. McDaniel, K. R. Fenton, K. R. Zavadil, T. Tyliszczak, W. Lai, and K. F. McCarty. Intercalation pathway in many-particle lifepo4 electrode revealed by nanoscale state-of-charge mapping. Nano letters, 13(3):866-872, 2013.

[7] J. Collins, G. Gourdin, M. Foster, and D. Qu. Carbon surface functionalities and sei formation during li intercalation. Carbon, 92:193-244, 2015.

[8] Y. R. Dougassa, J. Jacquemin, L. El Ouatani, C. Tessier, and M. Anouti. Viscosity and carbon dioxide solubility for lipf6, litfsi, and lifap in alkyl carbonates: lithium salt nature and concentration effect. The Journal of Physical Chemistry B, 118(14):3973-3980, 2014.

[9] M. Duduta, B. Ho, V. C. Wood, P. Limthongkul, V. E. Brunini, W. C. Carter, and Y. M. Chiang. Semi-solid lithium rechargeable flow battery. Advanced Energy Materials, 1(4):511-516, 2011.

[10] F. Y. Fan, W. H. Woodford, Z. Li, N. Baram, K. C. Smith, A. Helal, G. H. McKinley, W. C. Carter, and Y.-M. Chiang. Polysulfide flow batteries enabled by percolating nanoscale conductor networks. Nano letters, 14(4):2210-2218, 2014.

[11] C. R. Fell, L. Sun, P. B. Hallac, B. Metz, and B. Sisk. Investigation of the gas generation in lithium titanate anode based lithium ion batteries. Journal of The Electrochemical Society, 162(9):A1916-A1920, 2015.

[12] X. Han, M. Ouyang, L. Lu, J. Li, Y. Zheng, and Z. Li. A comparative study of commercial lithium ion battery cycle life in electrical vehicle: Aging mechanism identification. Journal of Power Sources, 251:38-54, 2014.

[13] K. B. Hatzell, M. Boota, and Y. Gogotsi. Materials for suspension (semisolid) electrodes for energy and water technologies. Chemical Society Re- 
views, 44(23):8664-8687, 2015.

[14] J. Jamnik and J. Maier. Treatment of the impedance of mixed conductors equivalent circuit model and explicit approximate solutions. Journal of The Electrochemical Society, 146(11):4183-4188, 1999.

[15] F. La Mantia, C. Wessells, H. Deshazer, and Y. Cui. Reliable reference electrodes for lithium-ion batteries. Electrochemistry Communications, 31:141$144,2013$.

[16] L. Lombardo, S. Brutti, M. A. Navarra, S. Panero, and P. Reale. Mixtures of ionic liquid-alkylcarbonates as electrolytes for safe lithium-ion batteries. Journal of Power Sources, 227:8-14, 2013.

[17] P. Lu, C. Li, E. W. Schneider, and S. J. Harris. Chemistry, impedance, and morphology evolution in solid electrolyte interphase films during formation in lithium ion batteries. The Journal of Physical Chemistry C, 118(2):896903, 2014.

[18] J. Nanda, J. Remillard, A. O’Neill, D. Bernardi, T. Ro, K. E. Nietering, J. Go, and T. J. Miller. Local state-of-charge mapping of lithium-ion battery electrodes. Advanced Functional Materials, 21(17):3282-3290, 2011.

[19] A. Narayanan, F. Mugele, and M. H. G. Duits. Mechanical history dependence in carbon black suspensions for flow batteries: a rheo-impedance study. Langmuir, 2017. (Chapter 3 of this thesis).

[20] T. Nishi, H. Nakai, and A. Kita. Visualization of the state-of-charge distribution in a licoo2 cathode by in situ raman imaging. Journal of The Electrochemical Society, 160(10):A1785-A1788, 2013.

[21] T. Ohzuku, A. Ueda, and N. Yamamoto. Zero-strain insertion material of li li1/3ti5/3 o-4 for rechargeable lithium cells. Journal of the Electrochemical Society, 142(5):1431-1435, 1995.

[22] T. J. Petek, N. C. Hoyt, R. F. Savinell, and J. S. Wainright. Slurry electrodes for iron plating in an all-iron flow battery. Journal of Power Sources, 294:620-626, 2015.

[23] Z. Qi and G. M. Koenig. A carbon-free lithium-ion solid dispersion redox couple with low viscosity for redox flow batteries. Journal of Power Sources, 323:97-106, 2016.

[24] G. Schramm. A practical approach to rheology and rheometry. Haake Karlsruhe, 1994.

[25] E. Ventosa, G. Zampardi, C. Flox, F. La Mantia, W. Schuhmann, and J. Morante. Solid electrolyte interphase in semi-solid flow batteries: a wolf in sheep's clothing. Chemical Communications, 51(81):14973-14976, 2015.

[26] J. Vetter, P. Novák, M. Wagner, C. Veit, K.-C. Möller, J. Besenhard, M. Winter, M. Wohlfahrt-Mehrens, C. Vogler, and A. Hammouche. Ageing mechanisms in lithium-ion batteries. Journal of power sources, 147(1):269- 
281, 2005.

[27] M. Youssry, L. Madec, P. Soudan, M. Cerbelaud, D. Guyomard, and B. Lestriez. Non-aqueous carbon black suspensions for lithium-based redox flow batteries: rheology and simultaneous rheo-electrical behavior. Physical Chemistry Chemical Physics, 15(34):14476-14486, 2013.

[28] M. Youssry, L. Madec, P. Soudan, M. Cerbelaud, D. Guyomard, and B. Lestriez. Formulation of flowable anolyte for redox flow batteries: Rheoelectrical study. Journal of Power Sources, 274:424-431, 2015.

[29] Y. Zhang, A. Narayanan, F. Mugele, M. C. Stuart, and M. H. Duits. Charge inversion and colloidal stability of carbon black in battery electrolyte solutions. Colloids and surfaces A: Physicochemical and engineering aspects, 489:461-468, 2016. (Chapter 2 of this thesis). 


\section{Chapter 6}

\section{Surface Layers}

Aditya Narayanan, Frieder Mugele, Michel Duits

The research described in this Chapter has been published as:

Electrochemically induced changes in $\mathrm{TiO} 2$ and Carbon films, studied with QCM-D, Advanced Energy Materials, 2019

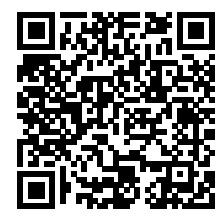




\subsection{Abstract}

Semi-solid fluid electrode-based battery (SSFB) and supercapacitor technologies are seen as very promising candidates for grid energy storage. However, unlike for traditional batteries, their performance can quickly get compromised by the formation of poorly conducting Solid Electrolyte Interphase on the particle surfaces. In this work we examine SEI film formation in relation to typical electrochemical conditions, by combining Cyclic Voltammetry (CV) with Quartz Crystal Microbalance-Dissipation monitoring (QCM-D). Sputtered layers of typical SSFB materials like Titanium dioxide $\left(\mathrm{TiO}_{2}\right)$ and Carbon, immersed in alkyl carbonate solvents, are cycled to potentials of relevance to both traditional and flow systems. Mass changes due to Lithium intercalation and SEI formation are distinguished by measuring the electrochemical current simultaneously with the damped mechanical oscillation. Both the $\mathrm{TiO}_{2}$ and amorphous Carbon layers show a significant irreversible mass increase on continued exposure to (even mildly) reducing electrochemical conditions. Studying the small changes within individual charge-discharge cycles, $\mathrm{TiO}_{2}$ shows mass oscillations, indicating a partial reversibility due to lithium intercalation (not found for Carbon). Viscoelastic signatures in the $\mathrm{MHz}$ frequency regime confirm the formation and growth of a soft layer, again with oscillations for $\mathrm{TiO}_{2}$ but not for Carbon. All these observations are consistent with irreversible SEI formation for both materials, and reversible Lithium intercalation for $\mathrm{TiO}_{2}$. Our results highlight the need for careful choices of the materials chemistry and a sensitive electrochemical screening for fluid electrode systems. 


\subsection{Introduction}

Novel solutions to grid energy storage such as Semi Solid Flow Batteries (SSFBs) and Flow Super-capacitors (FSCs) have gained strong interest in the recent past $^{12,13,18,28,31,37}$. Most of these systems store energy in electrode slurries of active and conductive particles suspended in an electrolyte. Here, the active particles store charge through capacitance or redox reactions, while the conductive particles form extended networks that electronically wire the active particles to current collectors. The liquid-suspended state of the particles offers great flexibility in operating the battery, but also poses new challenges. The reason lies in the dynamic structure of the fluid: imposed or thermal motions lead to rejuvenation of the particle assembly and hence also of the individual particle contacts. This repeated making and breaking of particle contacts causes the entire surface of each particle to be exposed to electrochemically induced changes. Since these surface modifications are incorporated in the (re-) assembled particle aggregates or networks, vital processes in SSFBs or FSCs can get compromised. ${ }^{21-23,50}$

It is well known that during electrochemical cycling, surface layers referred to as Solid Electrolyte Interphase (SEI) can form on solid electrode components (see e.g. ${ }^{26}$ for a recent review). These layers are formed by the decomposition of the electrolyte solution and impurities on the anode ${ }^{2,17,26,45}$. The SEI is typically ionically conductive and electronically insulating. SEI is a critical component of rechargeable Lithium ion batteries as it passivates the anode and thus allows operation at potentials that exceed the thermodynamic stability window of the electrolyte. While essential for solid Lithium ion batteries, such layer formation has been identified as potentially very detrimental to semi solid flow systems ${ }^{23,44}$. It increases the interparticle resistance (and overall cell resistance) and decreases 
the mechanical strength of the conductive particle network. A mitigation strategy suggested by some researchers is, to avoid SEI formation altogether by using materials that operate within the electrochemical stability window of the electrolytes $^{12,13,44}$. An example of this is the operation of Lithium Titanate (LTO) or $\mathrm{TiO}_{2}$ based SSFB anodes (Lithium intercalation potential: $\sim 1.5 \mathrm{~V}$ vs. $\mathrm{Li} / \mathrm{Li}^{+}$) at voltages above $1.0 \mathrm{~V}$ in alkyl carbonate solvents with $\mathrm{LiPF}_{6}{ }^{43}$. However, recent works have shown that significant detrimental effects due to layer formation can occur even within these conservative operating potential windows 22,23 .

While there have been many studies on the formation and optimization of SEI, the scope of almost all have been traditional solid Lithium batteries (e.g. 4,19,20,24,25,46,47). A few studies have found indications of SEI layer formation within commonly used operating windows; however, these (relatively weak) effects were mostly not investigated further, probably because their relevance for traditional batteries was low $9,10,41$. However for SSFBs and FSCs, even thin SEI layers are potentially detrimental, as explained above. Examination for the presence and effect of thin SEI layers through the use of very (surface-) sensitive techniques is hence warranted for SSFB and FSC systems.

Electrochemical Quartz Crystal Microbalance with Dissipation monitoring (eQCMD) is an emerging technique ${ }^{39,40}$ which combines the recent extensions of QCM with electrochemical functionality (i.e. eQCM) ${ }^{3,8,19}$ and dissipation mode (i.e. QCM-D) ${ }^{32,33}$. The acoustic shear wave of a quartz resonator is used to probe the mass changes and viscoelastic characteristics of electrochemically deposited layers. The central part of the setup consists of a quartz disc (sensor area 79 $\mathrm{mm}^{2}$ ) enclosed between two metallic layers, of which one is additionally coated with the material of electrochemical interest. This electrode is immersed in elec- 


\subsection{INTRODUCTION}

trolyte within an electrochemical cell. While the cell potential is controlled, the mass and mechanical properties of the material deposited on the electrode are monitored by piezo-electrically exciting the crystal and measuring the 'ring down' of the damped mechanical oscillation. This method is very suitable for in-situ probing of the formation and characteristics of SEI layers ${ }^{11,16,36,47}$.

In the present work we use eQCM-D to investigate two materials which have been studied in the context of SEI formation and growth in solid Li batteries: $\mathrm{TiO}_{2}{ }^{5,48}$ and Carbon ${ }^{4,7,19,25,49}$. Both these materials are also broadly available in the form of colloidal particles and are considered for use in fluid electrodes ${ }^{12,18,43}$. We examine these materials in the form of sputtered layers, immersed in alkyl carbonate solvents with $1 \mathrm{M} \mathrm{LiPF}_{6}$ as in traditional Lithium-ion batteries. While exploring typical voltages for these systems, we focus in particular on the mildly reductive regime where SEI formation is often ignored. Four increasingly reductive voltage windows are addressed, and many charge-discharge cycles are performed in each of them.

Another specific focus of the present work lies in the mechanical compliance of the growing SEI layer. Extracting these from the eQCM-D data is not trivial, since they have to be obtained by fitting to a viscoelastic model. Standard models such as Voigt-Kelvin are likely to be over simplistic ${ }^{36}$ while for more sophisticated models the number of fit parameters should be kept appropriately small. We take these issues into account, and also examine the effect of slightly different model choices on the fitted masses and compliances. Building on the outcome of our analysis, we find semi-quantitative trends in the compliances, and quantitative information on the mass evolution. Besides the gradual evolutions over many cycles, we also examine the changes within single charge-discharge cycles. As 
we will show, the latter allows us to obtain additional insights from correlations between the elastic compliance, the mass and the amount of injected charge.

\subsection{Materials and Methods}

\subsubsection{Materials}

Propylene Carbonate (PC), Ethylene Carbonate (EC) and Dimethyl Carbonate (DMC) were obtained from Sigma-Aldrich (anhydrous, 99\%+ purity). $\mathrm{LiPF}_{6}$ (98\% purity) and Lithium foil (99.9\% purity) were purchased from Alfa Aesar. All chemicals were stored in an Argon filled MBraun LABstar glovebox with $\mathrm{H}_{20}$ and $\mathrm{O}_{2}$ concentrations below $0.5 \mathrm{ppm}$. The electrolytes used in the experiments were $1 \mathrm{M} \mathrm{LiPF}_{6}$ salt solutions in $\mathrm{PC}$ (viscosity: $8 \mathrm{mPa} . \mathrm{s}$ ), unless mentioned otherwise.

\subsubsection{Sensor Preparation}

Gold coated $5 \mathrm{MHz}$ quartz QCM sensors (Renelux Crystal) were used. After cleaning they were first coated with $200 \mathrm{~nm}$ of sputtered copper. Amorphous Carbon films were then deposited by DC magnetron sputtering using a graphite (99.999\%) target disk in an Argon plasma at a pressure of $6.6 \mu$ bar. The film thickness was controlled via the deposition time, and verified to be $\sim 77 \pm 2 \mathrm{~nm}$ using ellipsometry. $\mathrm{TiO}_{2}$ films were prepared by reactive sputtering (DC power $500 \mathrm{~W})$ of a titanium $(99,999 \%)$ target . After the deposition of a few nanometers of pure Ti to aid adhesion, also Oxygen gas ( 6 standard $\left.\mathrm{cc} \mathrm{m}^{-1}\right)$ was admitted to the chamber, to deposit an amorphous $\mathrm{TiO}_{2}$ layer. Via control over the deposition time a thickness of $\sim 100 \mathrm{~nm}$ was obtained, with a peak-to-peak roughness $<10$ nm, as measured with AFM. A post-annealing step was performed at $500 \mathrm{C}$ in 
an atmospheric environment for 8 hours to crystallize the amorphous film to anatase $^{30}$.

\subsubsection{EQCM Cell}

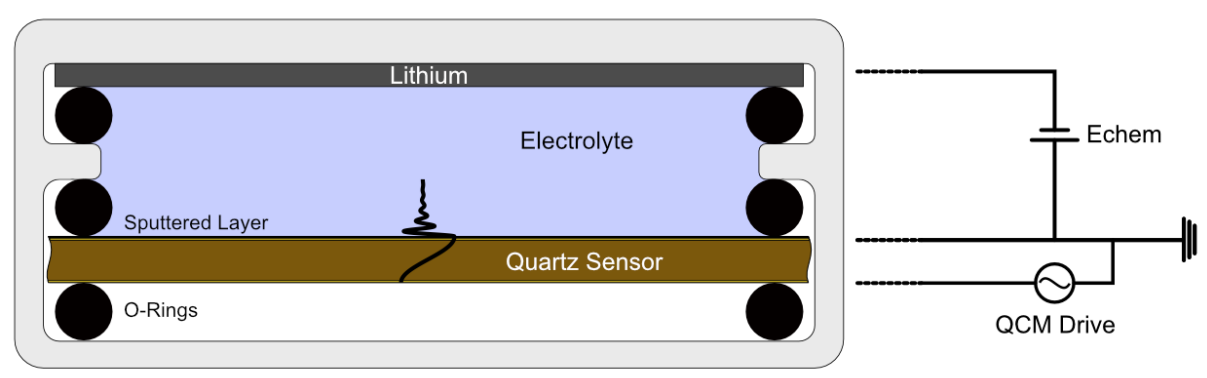

Figure 6.1: Cartoon of eQCM cell. See text for further details.

A custom eQCM cell (Fig. 6.1) was designed, to enable studying Lithium-based electrochemistry in alkyl carbonate solvents. The cell uses a modified bottom holder of the commercial QSense EQCM cell (Biolin Scientific). The top part was fabricated out of PEEK and has channels to allow introduction of the electrolyte into the chamber via PEEK microfluidic connections. Directly above the fluid chamber is a replaceable Lithium foil which acts as a counter electrode. The Lithium foil and quartz sensor are sealed against the chamber with identical Orings to keep their electrochemically active area the same $\left(1.15 \times 10^{-4} \mathrm{~m}^{2}\right)$. The eQCM cell was assembled in the glove box, completely sealed, and then connected to the QCM-D (Biolin Scientific) and electrochemical potentiostat outside. The electrode with the sputtered layer was at the common ground of both instruments. 


\subsection{QCM-D Analysis}

\subsubsection{Theory}

The QCM-D measures, through a ring down procedure, a complex resonance frequency $\tilde{f}$ that contains a real frequency $f$ and a half-bandwidth $\Gamma$. We note here that the Q-sense QCM-D instrument outputs a dissipation factor which is a frequency normalized bandwidth $(D=2 \Gamma / f)$.

$$
\tilde{f}=f+i \Gamma
$$

The instrument measures these values for multiple odd overtones $n$. In our experiments, we only consider the overtones from 5 to 13 as these are least affected by the mounting which can vary due to effects like O-ring swelling, etc. ${ }^{14}$

As the QCM sensor is loaded (with a film, e.g. SEI), its complex resonance frequency shifts. If the shift is small compared to the reference resonance frequency, we can use the small load approximation (Eq. 6.2) which states that the shift is proportional to the load impedance $\left(\tilde{Z}_{\text {Load }}\right)$ on the crystal surface: ${ }^{14}$

$$
\frac{\tilde{f}}{f_{0}} \approx \frac{-i}{\pi Z_{q}} \tilde{Z}_{\text {Load }}
$$

Where $f_{0}$ is the fundamental frequency of the unloaded crystal and $Z_{q}$ the acoustic shear wave impedance of the AT cut quartz. For a thin film in liquid, using the small load approximation and referencing the shifts to the unloaded* immersed crystal, the complex shift is given by: ${ }^{14}$

*sputtered layers are considered as part of the 'unloaded' crystal since the acoustic wave impedances of the sputtered layers are close to quartz as compared to layers deposited during the experiment. 


$$
\frac{\tilde{f}}{f_{0}} \approx \frac{-2 n f_{0} m_{f}}{Z_{q}}\left[1-2 \pi i n \frac{\tilde{J}_{f}(\omega)}{\rho_{f}} f_{0} \rho_{l} \eta_{l}\right]
$$

Where $n$ is the overtone number, $m$ a mass, $\rho$ a mass-density, $\tilde{J}_{f}(\omega)$ the complex compliance at the frequency $\omega=2 \pi n f$, and $\eta$ a viscosity. The subscript $f$ represents the deposited film while $l$ refers to the liquid.

The complex compliance of the film $\tilde{J}_{f}$ is defined as

$$
\tilde{J}_{f}(\omega)=J^{\prime}(\omega)-i J^{\prime \prime}(\omega)
$$

Where $J^{\prime}$ and $J^{\prime \prime}$ are the frequency dependent elastic and viscous compliances. We henceforth drop the $f$ subscript for the viscoelastic properties of the film. The pre-factor in Eq. 6.3 has the same form as the Sauerbrey equation (referenced to the bare crystal in liquid). Indeed, if the film has a zero viscoelastic compliance (a perfectly rigid film), the equation reduces to the Sauerbrey result. It can also be seen from Eqs. 6.3 and 6.4 that $J^{\prime}$ affects the half-bandwidth $\Gamma$, while $J^{\prime \prime}$ affects the resonance frequency $f$.

\subsubsection{Model Fitting}

In principle the above equations can be solved (using all overtones) to obtain the mass and viscoelastic properties, however as the mass is not known a priori, this is not trivial. To reduce the number of fit parameters, we impose relations between the viscoelastic compliances at different frequencies. Since viscoelastic relaxation spectra inherently show a very gradual frequency dependence and the QCM-D probes only one decade in frequency, we can assume power law forms for both compliances. This is approach is hardly restrictive, and certainly preferable 
to overly simplistic models such as Kelvin-Voigt, which ignore the possibility that a material can have multiple relaxations. The power law compliances are given by:

$$
\begin{gathered}
J^{\prime}(\omega)=J_{r e f}^{\prime}\left(\frac{f}{f_{\text {ref }}}\right)^{\beta^{\prime}} \\
J^{\prime \prime}(\omega)=J^{\prime \prime}{ }_{\text {ref }}\left(\frac{f}{f_{\text {ref }}}\right)^{\beta^{\prime \prime}}
\end{gathered}
$$

Where the reference frequency is chosen close to the middle of the operating range $(35 \mathrm{MHz})$. The viscoelastic exponents are determined by the spectral distance between the measurement frequency range and the layer material's intrinsic relaxation frequencies. Assuming that no drastic changes in the material composition occur during the growth of the SEI layer, the exponents $\beta^{\prime}$ and $\beta^{\prime \prime}$ can be considered as constant throughout the entire experiment. Since the elastic and viscous compliances are interrelated by the Kramers-Kronig relations, $\beta^{\prime}$ must lie between -2 and 0 and $\beta^{\prime \prime}$ between -1 and $1 .^{14,42}$

Making reasonable estimates for $\rho_{f}$, we are left with 5 unknown parameters $\left(m_{f}, J_{r e f}^{\prime}, \beta^{\prime}, J^{\prime \prime}{ }_{r e f}, \beta^{\prime \prime}\right)$ and 10 relations for 5 overtones. A method is now required to obtain reasonable initial guesses for these parameters, to avoid trapping in local minima during fitting. A first estimate for $m_{f}$ is obtained by fitting the Sauerbrey equation $\left(\tilde{J}_{f}=0\right)$ for all overtones. Using this value in the complex part of Eq. 6.3, we can directly calculate estimates for $J^{\prime}(\omega)$ from the various half bandwidth shifts, and thus ${J^{\prime}}_{r e f}$ and $\beta^{\prime}$. While it is tempting to use the same method to approximate $J^{\prime \prime}(\omega)$, this would give erroneous results since $m_{f}$ was already approximated from the frequency shifts. Instead we first assume a constant (i.e. frequency independent) $J^{\prime \prime}$, estimated from the real part of Eq. 
6.3 for the reference frequency. Using the above initial guesses, we systematically explore all combinations of viscoelastic exponents $\beta^{\prime}$ and $\beta^{\prime \prime}$ while allowing $m_{f}$, $J^{\prime}{ }_{r e f}$ and $J^{\prime \prime}{ }_{r e f}$ to vary as fit parameters.

In this scheme, the time dependent properties of the layer are found by fitting $m_{f}, J^{\prime}{ }_{r e f}$ and $J^{\prime \prime}{ }_{r e f}$ over the course of the entire experiment (i.e. voltage-time profile) while keeping $\beta^{\prime}$ and $\beta^{\prime \prime}$ fixed. This fitting operation is performed for 100 different $\left(\beta^{\prime}, \beta^{\prime \prime}\right)$ combinations, as obtained by varying each exponent over the entire possible range, in steps of 0.2. The total mean squared error (TMSE) of the fit is then mapped versus $\beta^{\prime}$ and $\beta^{\prime \prime}$, after which the $\left(\beta^{\prime}, \beta^{\prime \prime}\right.$ combination that produces the minimum TMSE is chosen to obtain the time-dependent $m_{f}$, $J^{\prime}{ }_{r e f}$ and $J^{\prime \prime}{ }_{r e f}$ signals. A detailed description of the model fitting, along with a discussion of the uncertainty in the fitted parameters can be found in the Supplementary Information (SI).

We mention here that we use in fact a slightly modified version of Eq. 6.3; a third order perturbation analysis ${ }^{14}$ equation that contains (small) corrections to deal with violations of the small load approximation. Frequency and halfbandwidth shifts that were large enough to necessitate this correction were mainly found in the last (i.e. forth) stage of the experiment. The SI (Section 6.7.1) contains a brief description of this equation. Also the effects of SEI multilayers and roughness are briefly discussed. 


\subsection{Results and Discussion}

\subsubsection{Measured QCM-D signals and model fits}

In Fig. 6.2 the direct output signals from the QCM-D instrument, i.e. the frequency- and half bandwidth-shifts of the various overtones for the 4 different voltage regimes are shown (solid lines and circles). The current responses of the Cyclic Voltammograms are shown in the SI (Fig. 6.17). The experiments on the different materials are grouped for comparison, as $\mathrm{TiO}_{2}$ is a good intercalation material while amorphous Carbon is not.

It is clear that for both materials, over timescales much longer than one $\mathrm{CV}$ cycle, the (kHz range) negative frequency shift gets progressively larger, with sudden changes in the slope at the points where a more reductive voltage regime is entered. The (Hz range) half-bandwidth shift also increases with time for most regimes; the only exception being the amorphous Carbon in the most reductive regime. A noticeable difference between the $\mathrm{TiO}_{2}$ and the Carbon is that the former material shows very clear oscillations in $\Delta f$ and $\Delta \Gamma$, in phase with the charge-discharge cycles (as we will see below).

Also shown in Fig. 6.2 are model fits to the data. All frequency-shift differences are below $50 \mathrm{~Hz}$ and half-bandwidth-shift differences are below $8 \mathrm{~Hz}$ (see SI Figs. 6.11-6.12). The root-mean-squared fitting error was below $25 \mathrm{~Hz}$ for all overtones, which corresponds to a Sauerbrey mass 'fitting error' of around $5 \times 10^{-7} \mathrm{~kg} \mathrm{~m}^{-2}$. This error is of the same magnitude as the measurement noise. The goodness of fits and trends were not affected by small variations of the grid fit parameters $\left(\beta^{\prime}, \beta^{\prime \prime}\right)$. See the SI for a discussion about the accuracy and robustness of the fitting. 

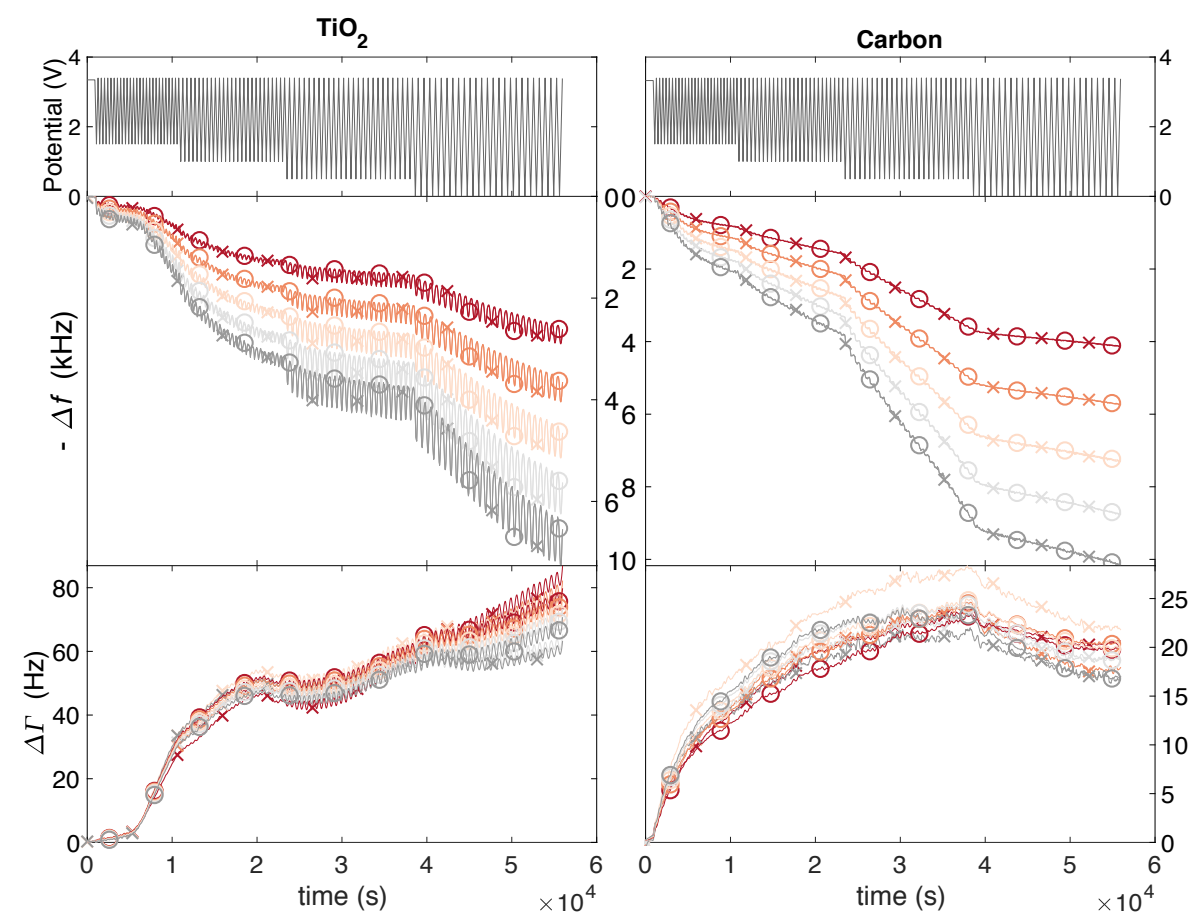

Figure 6.2: Applied cell potential (top), frequency shift (middle) and halfbandwidth shift (bottom) for $\mathrm{TiO}_{2}$ (left) and Carbon (right) electrodes when cycled over four voltage windows of 25 cycles each. The various odd overtones (5-13) are shown in different colors. Both experimental data and model fits are shown as solid lines, where the half-bandwidth shifts have been Savitzk-Golay (SG) smoothed to reduce noise. Markers have been added: (o) for experiments and (x) for fits. Note that the frequency shifts are in $\mathrm{kHz}$, while it is $\mathrm{Hz}$ for the half-bandwidth shifts. 


\subsubsection{Global changes in mass and viscoelastic compliance}

Assuming that the shifts are due to a uniform viscoelastic layer and using the model described in Section 6.4.2, we obtain the QCM areal mass density $\left(m_{f}\right)$ shown in Fig 6.3. Here $m_{f}$ is plotted along with a theoretical areal Lithium mass density $\left(m_{e}\right)$. To find $m_{e}$, we integrate the current and convert the obtained faradic charge to an areal mass of Lithium atoms. We henceforth refer to the areal mass as just the mass.
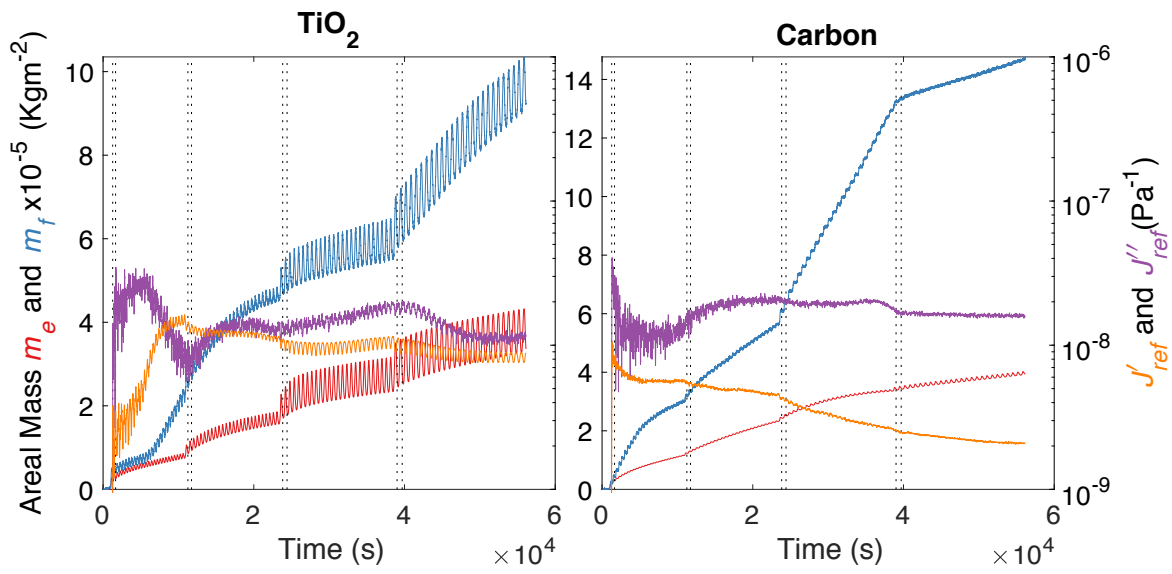

Figure 6.3: $m_{e}$ (red), $m_{f}$ (blue), $J^{\prime}{ }_{r e f}(35 M H z)$ (orange) and $J^{\prime \prime}{ }_{r e f}(35 M H z)$ (purple) derived from the model in Section 6.4.2 for the experiment in Fig. 6.2. Dashed vertical lines highlight the cycles examined in Fig 6.4. Viscoelastic compliances have been SG smoothed and share the same scale for both experiments.

For both materials, $m_{f}$ and $m_{e}$ are observed to grow irreversibly with time. In the first few cycles of the initial (i.e. least reductive) regime, the two signals are similar but well before the end of that regime, $m_{f}$ gets significantly larger than $m_{e}$, and the difference keeps growing. The irreversible mass increases clearly indicate the formation of a Solid Electrolyte Interphase. Possibly some irreversible 


\subsection{RESULTS AND DISCUSSION}

Lithium intercalation takes place too.

It is remarkable that for both the $\mathrm{TiO}_{2}$ and the Carbon, significant irreversible layer growth is found even in the least reducing voltage window of $1.5 \mathrm{~V}$, well within the (for traditional Li batteries) accepted operating window of the electrolyte. Assuming a layer density of $1500 \mathrm{~kg} \mathrm{~m}^{-2}$, we find that the layers on both surfaces are already several nm thick after a few CV cycles (the consequences of choosing a different layer density are discussed in SI sect. 6.7.1; there we show that $m_{f}$ remains essentially unaffected while $J^{\prime}{ }_{r e f}$ and $J^{\prime \prime}$ ref change but their trends do not). By the end of the 25 cycles a thickness of $\sim 20 \mathrm{~nm}$ is reached. While such thin layers are of minimal consequence in solid Lithium ion batteries, flow systems can be strongly affected by thin layers (especially if the latter are insulating). Electron conduction paths between current collectors and active particles generally involve a huge number of inter-particle contacts. When the particles are very close (as in a gel network), conduction is governed by either fluctuation-induced tunneling or by the limiting intrinsic electronic conductivity of the separating medium ${ }^{34,35,38}$. In the respective cases, there is an inverse exponential or inverse dependence of the conductivity on the insulating gap length. In traditional solid electrode systems, the inter-particle contacts themselves are static. Therefore, most contacts are exposed to little electrolyte or protected by the binder. The overall electrode resistance is then only slightly affected by thin SEI. In SSFB systems however, particle contacts are refreshed due to restructuring by flow. The incorporation of many of these thin insulating layers in the conduction path can thus have a dramatic effect on the electrode resistance. ${ }^{23}$

As a general trend, on continuing the cycling inside a given voltage window, the layer properties tend to saturate (i.e. the SEI growth rate tends to decrease). 
When a new, more reducing window is started, the growth rate generally increases again, presumably due to a greater potential drop over the pre-existing layer and/or the activation of new reactions. One exception can be found for the Carbon sample, where the growth rate strongly decreases right at the beginning of the $0 \mathrm{~V}$ window. Here, the SEI layer is calculated to be around $100 \mathrm{~nm}$ thick, which is still well below the penetration depth (the maximum sensing depth) of the shear wave through it $(\sim 900 \mathrm{~nm}$ at $65 \mathrm{MHz})$. The slowing down of the layer growth is thus real. This might be due to an almost complete passivation of the Carbon electrode.

Fig. 6.3 also shows the global variation of the elastic and viscous compliances, which are of comparable magnitude. In general, cycling at more reducing voltages diminishes both compliances, i.e. stiffens the layer. During the initial cycles when the layers are still very thin, the contribution of the compliance term to Eq. 6.3 is too small to resolve it with accuracy.

\subsubsection{Changes per Voltage cycle}

The second cycle of each voltage window of the experiment in Fig. 6.2 is plotted as a cyclic voltammogram in Fig 6.4. In addition to the current, also the changes in $m_{e}$ and $m_{f}$ (referenced to the beginning of the cycle) are plotted. The signals corresponding to $\Delta m_{f}$ are relatively noisy, as the mass changes within each cycle are small and close to the noise limit $\left(\sigma \approx 5 \times 10^{-7} \mathrm{kgm}^{-2}\right)$ of the QCM-D for this system.

In the $\mathrm{TiO}_{2}$ sample, both mass changes are largely reversible within a single cycle. Below $\sim 2.5 \mathrm{~V}$ the current starts to increase and consequently also $m_{e}$. $m_{f}$ closely follows the trend of $m_{e}$, suggesting that most of its growth is due to 
$\mathrm{TiO}_{2}$
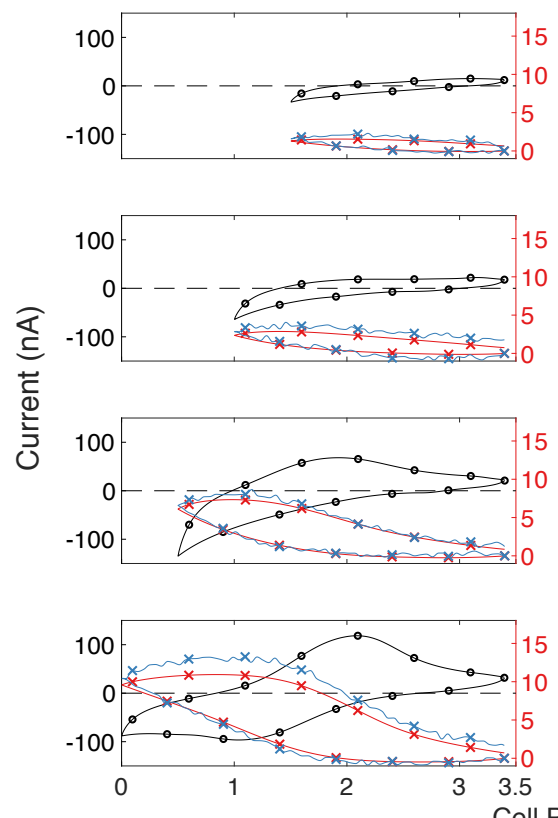

Carbon
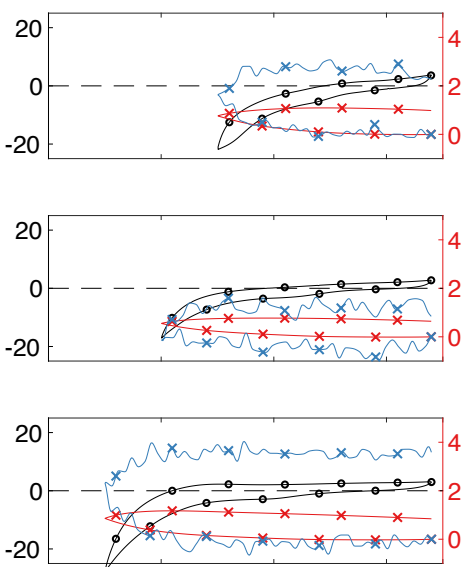

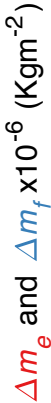

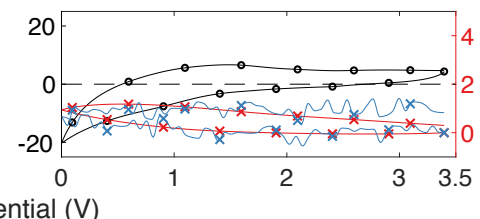

Figure 6.4: CVs with current (black o) and change in $m_{e}$ (red $x$ ), $m_{f}$ (blue $x$ ) for the second cycle of each window (highlighted in Fig. 6.3). In each cycle the potential vs. $\mathrm{Li} / \mathrm{Li}^{+}$starts at $3.4 \mathrm{~V}$ and returns there after a clockwise trajectory in the I-V plot. Also the $m$-V curves run clockwise. Left: $\mathrm{TiO}_{2}$, Right: Carbon. For each sample the axis scales are kept the same to allow comparison.

Lithium intercalation. On the reverse half cycle, the reduction in $m_{e}$ is also closely followed by $m_{f}$, again pointing at Lithium (de-)intercalation as the dominant process. At the end of the cycle there is a small overall positive growth in both the $m_{e}$ and $m_{f}$, in agreement with the trend over multiple cycles shown in Fig. 6.3. This net effect per cycle is thus significant, and is attributed to irreversible SEI growth, along with a possible contribution from irreversible intercalation. Of 
note is how close the values and trends of the changes of $m_{f}$ and $m_{e}$ are, lending confidence to the accuracy of the analysis.

For the Carbon sample, the currents and mass changes are significantly smaller than for $\mathrm{TiO}_{2}$, causing the $m_{f}$ signals to be even more noisy. The behavior is qualitatively different from $\mathrm{TiO}_{2}$ : while both $m_{f}$ and $m_{e}$ grow upon reducing the voltage, in the reverse part of the cycle only a slight decrease in $m_{e}$ is seen while $m_{f}$ appears to remain constant (or even grow a little). This clearly suggests that the growth of $m_{f}$ is not determined by reversible Lithium intercalation.

So far, a cycle has referred to the cell starting at $3.4 \mathrm{~V}$, going down to the low voltage boundary $(1.5 / 1.0 / 0.5 / 0.0 \mathrm{~V})$ and then back up to the initial voltage. However, in this potential based scheme, the growth or shrinkage of a layer is not complete at the end of a cycle; see Fig. 6.5. The layer changes the least when the current is zero. We henceforth use every third current zero crossing to demarcate cycles.

We now look at the changes in the viscoelastic compliance and the mass during the second cycle of each window (Fig. 6.6). Again, the changes are referenced to the beginning of the cycle. For the first voltage window, for both samples, the compliance signals are very noisy and difficult to extract, even though a change in mass is easily detected. This is because the layer is still extremely thin $(\sim 2$ $\mathrm{nm})$.

For the subsequent voltage widows, the $\mathrm{TiO}_{2}$ sample shows a noticeable change in the elastic compliance $J^{\prime}$ when the mass changes. When Lithium is intercalated into a host, it can change the structure and consequently the mechanical properties of the latter. Lithium intercalation is known to decrease the compliance of metal oxides and layered materials, while it increases the compliance of 

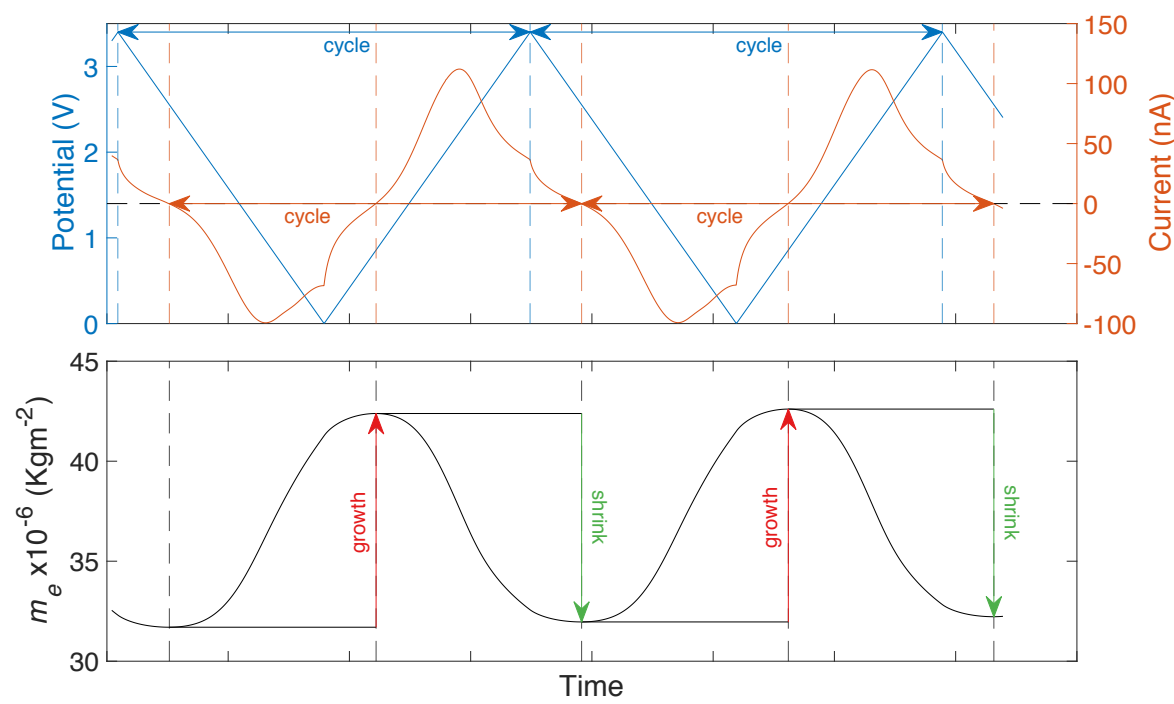

Figure 6.5: Top: Typical cell graph of potential and current versus time (here for $\mathrm{TiO}_{2}$ in the most reductive voltage window; 340 s per cycle). The blue arrows indicate voltage-based cycles while the orange ones designate current-based cycles. Bottom: The change in $m_{e}$ with the growth (red arrow) and shrinkage (green arrow).

materials it alloys with ${ }^{29}$. The changes in the elastic compliance may thus be related to Lithium intercalation. At the end of the cycles for $\mathrm{TiO}_{2}$, the elastic compliance almost returns to its original value after de-intercalation. However, the recovery is not full. This can be seen from the general reduction of the elastic compliance as cycling is continued (see Fig. 6.3).

We add here that for the $\mathrm{TiO}_{2}$ sample, the raw data show a strong change in the half-bandwidth shift (which is directly proportional to the layer's elastic compliance and mass; see Eq. 6.3) in the two distinct stages of the charge-discharge cycle. Here, the bandwidth shift decreases despite the layer mass increasing, and 


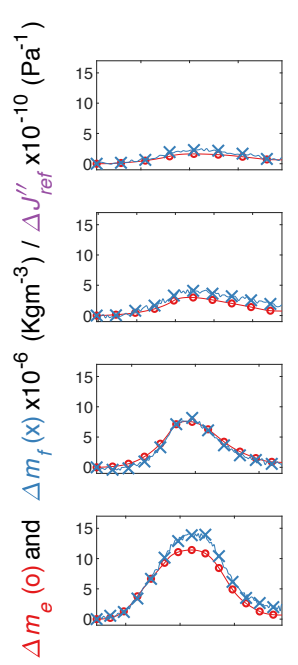

$\mathrm{TiO}_{2}$
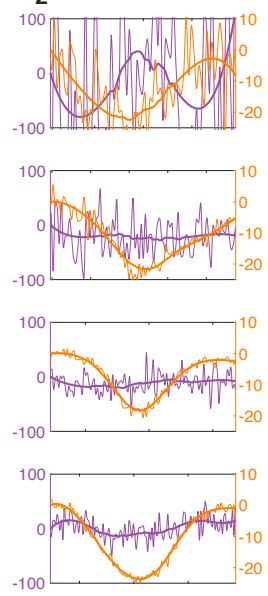

Carbon
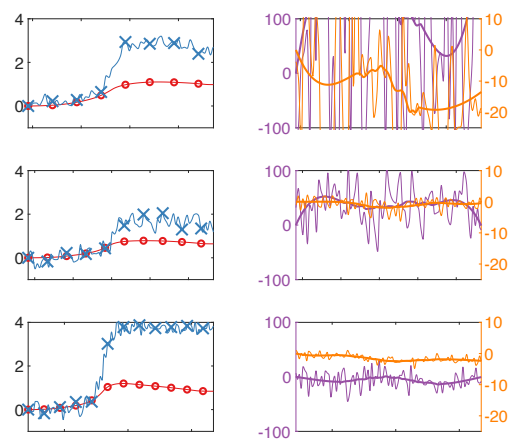

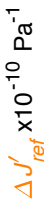

Figure 6.6: First column: Plots of the change in in $m_{e}$ (red o), $m_{f}$ (blue $x)$. Second column: the viscoelastic compliances $J^{\prime}{ }_{r e f}(35 \mathrm{MHz})$ (orange) and $J^{\prime \prime}{ }_{r e f}(35 \mathrm{MHz})$ (purple) versus time for the second cycle of each voltage window of the experiment in Fig. 6.2. For the compliances both the raw (light thin lines) and $S G$ smoothed (dark thick lines) data are shown. Left: $\mathrm{TiO}_{2}$ Right: Carbon.

vice versa. Thus, the trend in the compliance is not a spurious fitting effect induced by the changing layer mass.

A second observation for the $\mathrm{TiO}_{2}$ sample is that $\Delta m_{f}$ is not precisely equal to $\Delta m_{e}$, as would be expected for a perfectly reversible Lithium intercalation. In particular the amplitude of $\Delta m_{f}$ per half cycle is somewhat larger than $\Delta m_{e}$. While this may be due to effects like a slightly reversible SEI (eg. due to dissolution $)^{6,23,27,51}$, it is difficult to quantify due to the sensitivity to the normalization of the lithium mass to the active area. This area is not very precisely known due to effects like o-ring compression and swelling. 
For the Carbon sample, despite significant mass changes, the changes in compliance are only minor (while still leading to a small overall decrease per cycle, see Fig. 6.3). Lithium insertion in layered Carbons is expected to have a much stronger effect on the compliance than that in metal oxides. Our findings thus imply that the mass change of the Carbon layer is mainly due to SEI growth and not irreversible Lithium insertion.

\subsubsection{Correlation between changes in mass and compliance}

We extend our analysis of the charge-discharge cycles by correlating the net changes in $m_{f}, m_{e}$ and $J^{\prime}(35 M H z)$ per half-cycle. Defining the cycles as in Fig. 6.5, $\Delta m_{e}$ will inherently be positive in the first half-cycle, and negative in the second one. Accordingly, we define the first half-cycle as 'growth' and the second one as 'shrinkage' (which thus refers strictly to $m_{e}$ ). To facilitate comparison of the magnitudes, we take the negative of $\Delta m_{e}$ in the shrinkage regime and denote it as $\Delta_{s} m_{e}$. For notational consistency, $\Delta m_{e}$ in the growth regime is just replaced by $\Delta_{g} m_{e}$. Similar definitions are applied to $\Delta m_{f}$ and $\Delta J^{\prime}$. It is noted here that $m_{f}$ does not have to change in the same direction as $m_{e}$; some cases were encountered where $m_{f}$ increased during 'shrinkage'. This was however an exception. For the far majority of our data, the diagnosis becomes more straightforward with these definitions. For example, comparisons between $\Delta_{g} m_{f}$ and $\Delta_{s} m_{f}$ (of the same cycle) allow examination of the reversibility of mass deposition, while differences between $\Delta_{g} m_{e}$ and $\Delta_{s} m_{e}$ indicate irreversible electron transfer.

Fig. 6.7 shows the (half-cycle) changes in $m_{f}, m_{e}$ and $J^{\prime}(35 \mathrm{MHz})$ for the entire experiment, for $\mathrm{TiO}_{2}$ (left panel) and Carbon (right panel). Important 
differences between the two materials become immediately evident. For the $\mathrm{TiO}_{2}$ sample, $\Delta m_{e}$ is similar to the total mass change, for nearly all half-cycles and irrespective of the (growth or shrinkage) stage. The near equality of $\Delta_{g} m_{e}$ and $\Delta_{s} m_{e}$ indicates a high degree of reversibility, which would also be expected for Lithium intercalation. The changes in $m_{f}$ are largely reversible as well, albeit less so than for $m_{e}$ (this causes the overall $m_{f}$ to grow over $m_{e}$ as seen in Fig. 6.3). Looking at the elastic compliance $J^{\prime}$, a strong anti-correlation with both $m_{e}$ and $m_{f}$ is observed. This corroborates Lithium intercalation.

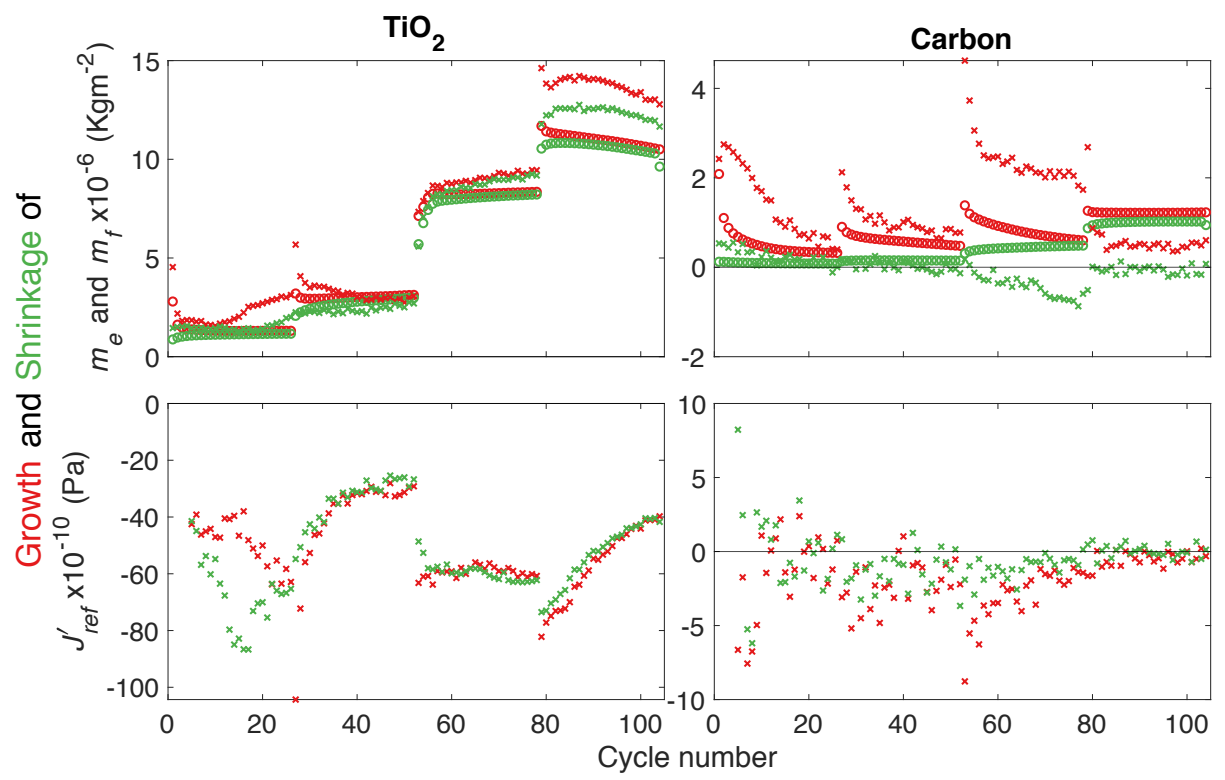

Figure 6.7: Cycle by cycle growth (red) and shrinkage (green) of Top: $m_{f}(x)$, $m_{e}$ (o) and Bottom: $J^{\prime}(35 \mathrm{MHz})$ for $\mathrm{TiO}_{2}$ (left) and Carbon (right). $J^{\prime}$ data are omitted for the first four cycles for both experiments. Note that the vertical scales for the Carbon sample are much smaller than for $\mathrm{TiO}_{2}$. 
For the Carbon sample the mass changes are significantly smaller than for the $\mathrm{TiO}_{2}$. Here the $\Delta m_{e}$ signals are generally smaller than $\Delta m_{f}$ (except for the most reductive voltage window where $\Delta m_{f}$ has become very small). The changes in $m_{f}$ are less reversible (compared to $m_{e}$ ) also for this material. Meanwhile the changes in elastic compliance fluctuate randomly. The $m_{f}$ and $m_{e}$ growths are weakly correlated while the shrinkages are not. These correlations (further illustrated in SI Fig. 6.16) indicate (more clearly now) that the majority of the mass changes in the Carbon sample are due to irreversible SEI formation in the growth stage $\left(\Delta m_{e}>0\right)$.

\subsubsection{Cycling of Carbon directly to $0 \mathrm{~V}$}

We continue our study of the Carbon system in $\mathrm{PC}$ with $1 \mathrm{M} \mathrm{LiPF} 6$, by cycling directly over the entire voltage range as done in traditional Lithium battery systems: between $3.4 \mathrm{~V}$ and $0 \mathrm{~V}$. This corresponds to the $4^{\text {th }}$ voltage window in the previous experiment, but with a different electrochemical history since we now start with pristine Carbon (not yet covered by SEI).

Fig. 6.8 shows the masses and visco-elastic compliances extracted from the QCM-D and current measurements, similar to Fig. 6.3. During the initial cycles, there is significantly larger ( $\sim$ an order of magnitude) SEI growth per cycle as compared to cycling the pristine Carbon in a $1.5 \mathrm{~V}$ voltage window (Fig. 6.3). Again, the gradual mass changes are much stronger for $m_{f}$ than for $m_{e}$, while this time there is almost no reversibility in $m_{f}$ within the cycle. As the cycling is continued, the layer growth slows down, while approaching a slightly larger thickness as compared to Fig. 6.3. The elastic compliance is very similar to that in Fig. 6.3, while the viscous compliance is higher now; this could indicate 


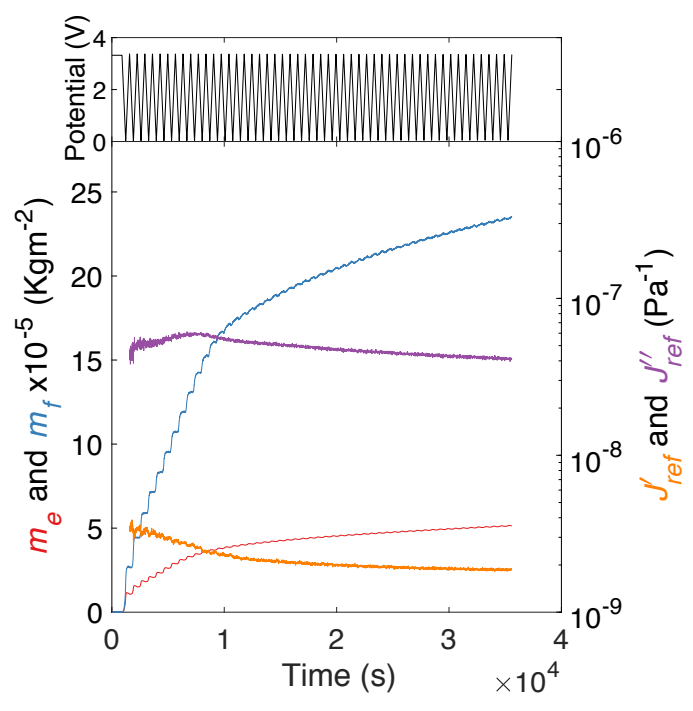

Figure 6.8: Carbon layer cycled to 0 V for 50 cycles. Top: Applied cell potential, Bottom: $m_{e}$ (red), $m_{f}$ (blue), $J^{\prime}(35 \mathrm{MHz})$ (orange) and $J^{\prime \prime}(35 \mathrm{MHz})$ (purple) versus time.

slight differences in the structure of the SEI formed in different voltage regimes. Importantly, cycling to strongly reducing voltages thus leads to quicker formation of an irreversible thick SEI, that is potentially catastrophic to flow systems.

\subsubsection{Cycling of Carbon in EC:DMC electrolyte}

In Fig. 6.9 we examine the behavior of the Carbon layer in a different electrolyte: EC-DMC with $1 \mathrm{M} \mathrm{LiPF}_{6}$, subsequently exploring two voltage windows: 3.4-1.5 $\mathrm{V}$ and 3.4-0 V. Though the growth in $m_{e}$ is similar to that in Fig. 6.3, the growth in $m_{f}$ is much larger. Thus, more SEI mass per unit charge is deposited in ECDMC as compared to PC. In addition, the viscoelastic compliances are much higher (along with the half bandwidth shift). Due to the latter, the QCM-D was 


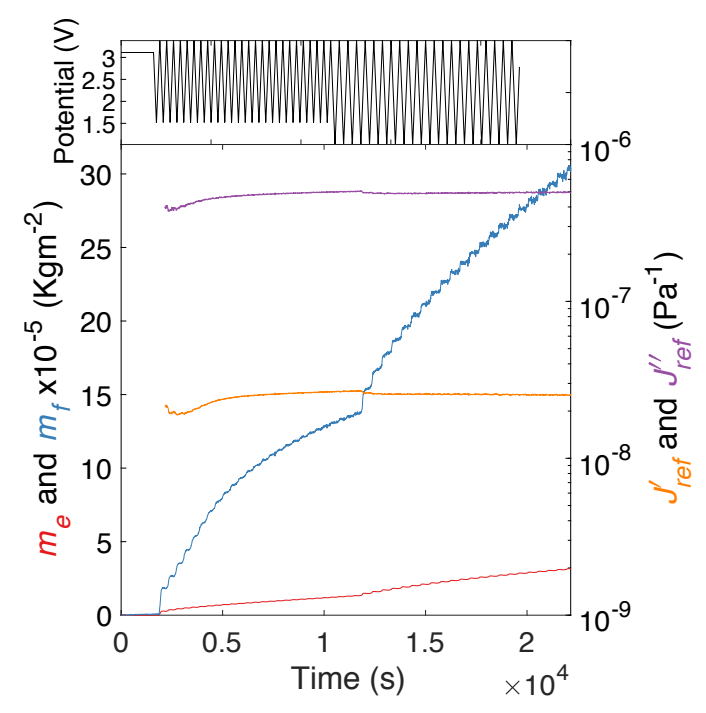

Figure 6.9: Carbon layer in EC DMC $1 M \mathrm{LiPF}_{6}$ cycled over two voltage windows. Top: Applied cell potential, Bottom: $m_{e}$ (red), $m_{f}$ (blue), $J^{\prime}(35 \mathrm{MHz})$ (orange) and $J^{\prime \prime}(35 \mathrm{MHz})$ (purple) versus time.

unable to track the experiment beyond the $49^{\text {th }}$ cycle. Assuming a similar mass density, the layer is around $15 \mathrm{~nm}$ thick after just the first cycle, and grows to over $200 \mathrm{~nm}$ at the end of the experiment. The partial reversibility in $m_{f}$ per cycle, evidenced by temporary mass decreases, largely disappears already after the first few cycles. Thus, in EC:DMC a permanent, 'fluffier' and heavier SEI than that in PC is formed at the same voltages. Such a layer, if insulating, would almost completely block electron transfer in a flow system. We note here that due to the much higher layer compliance; the model fits and outputs are very sensitive to the viscoelastic parameter choices unlike the previous experiments. 


\subsection{Conclusions}

Using the sensitive eQCM-D we have shown the formation of surface layers on Carbon and $\mathrm{TiO}_{2}$ surfaces (that emulate fluid electrode particles) in an alkyl carbonate solvent with dissolved $\mathrm{LiPF}_{6}$ during electrochemical cycling. Applying increasingly reductive potentials, the same gradual evolutions are observed for $\mathrm{TiO}_{2}$ and Carbon: an irreversible growth in deposited mass, and the formation of a viscoelastic layer. Focusing on the changes within individual charge-discharge cycles reveals additional information. For $\mathrm{TiO}_{2}$ the mass changes per cycle are dominated by reversible Li intercalation but the net effect of the cycle is a systematic mass growth, attributed to SEI. Also for Carbon a systematic mass growth is observed, but without clear indications for reversible intercalation. Correlating the changes in the mass and elastic compliance per cycle, we find a strong anti-correlation for $\mathrm{TiO}_{2}$ and a lack of correlation for Carbon. This underlines that while both intercalation and SEI growth lead to mass deposition, the former reduces the elastic compliance while the latter does not.

Our finding that even when cycling to $1.5 \mathrm{~V}$ vs Lithium ( a voltage considered to be within the operating window of most alkyl carbonate electrolytes) surface layers tens of nanometers thick form, is of significance for slurry-based electrodes, whose performance can be destroyed by thin insulating layers. Operating at less conservative voltages results in an even more SEI layer formation and layer thicknesses up to $\mathrm{O}(100 \mathrm{~nm})$. Our observations thus highlight the need for careful screening of systems for fluid electrode technologies, as they can be affected by SEI in operating regimes traditionally considered free of it. 


\subsection{Supplementary Information}

\subsubsection{Accuracy and robustness of the viscoelastic modeling.}

In this section we present some details of the model fitting, especially in relation to the viscoelastic exponents. We examine the $\mathrm{TiO}_{2}$ sample to check the modelling, and show some results for other systems later in the SI. Here, we point out an inherent limitation of the eQCM-D for studying SEI. The solid liquid interface is quite complex in Lithium batteries and is frequently comprised of multiple layers. Lithium intercalates into the outer material, thereby modifying it while an inner inorganic and outer organic SEI layer forms at the interface ${ }^{15}$. Additionally, these layers can vary spatially in homogeneity and porosity. While the acoustic shear waves of the QCM are sensitive to these factors, the reliance on a model makes these factors difficult to elucidate. This is further complicated by the fact that neither the structure nor the physical properties of the layer are known $a$ priori. In this section, in addition to exploring the robustness of our fits, we also explore the contributions these effects have on our system.

\section{Third order Perturbation Analysis}

$$
\frac{\Delta \tilde{f}}{f_{0}} \approx \frac{-2 n f_{0} m_{f}}{Z_{q}}\left[1-2 \pi i n \frac{\tilde{J}_{f}(\omega)}{\rho_{f}} f_{0} \rho_{l} \eta_{l}\right]
$$

The derivation of Equation 6.6 (Equation 6.3 in the main text) relies on the Small Load Approximation (SLA), which strictly applies only when layers are thin, and shifts are small ${ }^{14}$. 


$$
\frac{\Delta \tilde{f}}{f_{0}} \approx \frac{-2 n f_{0} m_{f}}{Z_{q}}\left[1-2 \pi i n \frac{\tilde{J}_{f}(\omega)}{\rho_{f}} f_{0} \rho_{l} \eta_{l}+(1+i) \sqrt{\frac{f_{0} \rho \eta_{l}}{n \pi Z_{q}^{2}}}+i \frac{2 \pi f_{0} n \rho_{l} \eta_{l}}{Z_{q}^{2}}\right]
$$

To cover for the regimes with thick SEI formation, we use a modified version of equation $6.3 / 6.6$ for our fitting. The third order perturbation equation ${ }^{14}$ (Eq. 6.7) contains (small) correction terms that avoid errors in the SLA due to large shifts. These errors usually show up in the n-dependence of $\tilde{J}_{f}(\omega)$. For our experimental data, we only see small differences in the model outputs of the two approaches. The third order perturbation equation mainly improves the fits for the half band-width shifts.

\section{Quality of overall fit, as a function of viscoelastic exponents}

We first focus on the effect of the viscoelastic exponents on the goodness of fit; the Root Mean Squared Error (RMSE):

$$
R M S E=\sqrt{\frac{1}{n} \sum_{n}\left(x_{\text {modeled }}-x_{\text {experimental }}\right)^{2}}
$$

Where $x$ is the frequency or half-bandwidth shift and the summation is over the whole experiment for overtones 5 through 13 . Fig. 6.10 shows maps of the RMSE of the frequency shift, bandwidth shift and the combination. During fitting, it is the RMSE of the combination that is minimized. As predicted by Equation $6.3 / 6.6$, the RMSE of the frequency shift is most dependent on $\beta^{\prime \prime}$ and that of the half-bandwidth shift on $\beta^{\prime}$. The overall RMSE shows a shallow minimum, implying that the choices of $\beta^{\prime}$ and $\beta^{\prime \prime}$ are not extremely critical (within a small range) to the fits. At the minimum, the RMSE is below $20 \mathrm{~Hz}$ which for our 
system at overtone 5 corresponds to a Sauerbrey mass of $6.5 \times 10^{-7} \mathrm{kgm}^{-2}$ (i.e. well below the found masses of o $10^{-5} \mathrm{kgm}^{-2}$. For the other overtones, the fitting error is similar.
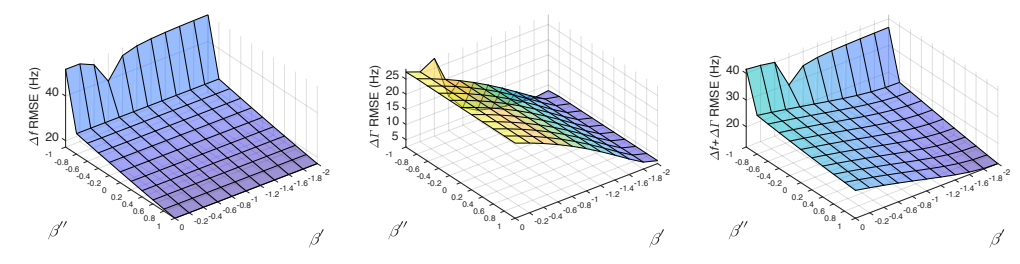

Figure 6.10: Maps of the Root Mean Squared Error (RMSE) for the grid-fit of the QCM data for $\mathrm{TiO}_{2}$ (all cycles in Fig. 6.2 of main text), allowing the exponents $\beta^{\prime}$ and $\beta^{\prime \prime}$ to vary independently within their physical bounds. Left: frequency, Center: half bandwidth shift and Right: combination

We now examine 25 model fits from the variation of the combination of $\beta^{\prime}$ and $\beta^{\prime \prime}$ around the best one. From the best combination, each exponent was varied in steps of 0.2 for a total range of 0.8 (40\% of total range) within the bounding values. Figures 6.11 and 6.12 show the experimental and modelled frequencyand half-bandwidth- shifts for these combinations.

From Fig. $6.11 \mathrm{~A}$ it is clear that there is a non-zero viscoelastic compliance, as the normalized frequency-shift curves do not superimpose. Fig. 6.12A shows that the half-bandwidth shifts almost superimpose, which implies that the layer's elastic compliance is nearly proportional to $n^{-2}$. This is consistent with the best fits being found for $\beta^{\prime}=-2$ and $\beta^{\prime \prime}=1$.

Fig. 6.11 also shows that for a reasonable range of the exponents, there is almost no visible difference in the modelled frequency-shifts. This implies that the viscous compliance makes only a minor contribution to the frequency shift. 

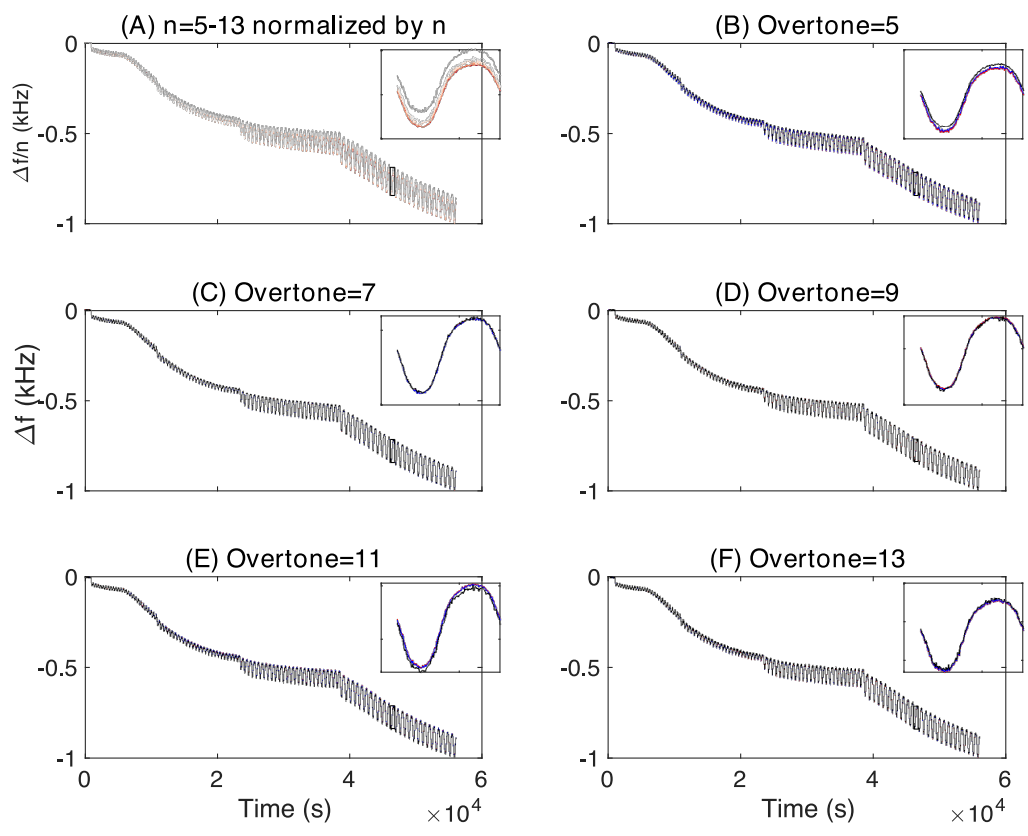

Figure 6.11: (A) Experimental frequency shifts normalized by overtone number. (B-F) Experimental frequency shift (black) and closest modeled shift (blue) and shifts for 24 nearby combinations of the viscoelastic exponents (red) for each overtone. Inset is a zoom of the boxed region.

As a consequence, the fitted layer mass should be largely decoupled from the fitted compliance and depend mainly on the frequency shift (approaching the Sauerbrey result: as $\tilde{J} \rightarrow 0$, the second term in the bracket of equation $6.3 / 6.6$ goes to zero).

While the RMSE values for the frequency and half bandwidth shift are similar, the differences in the modelled half-bandwidth curves for different exponents are larger. This is due to the proportional dependence of the half bandwidth shifts on the elastic compliance. 

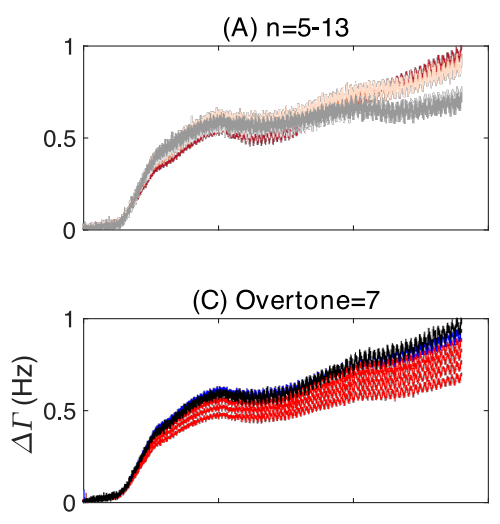

(E) Overtone $=11$

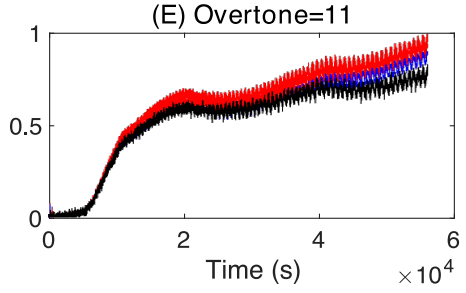

(B) Overtone $=5$

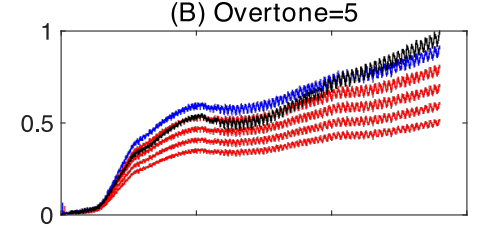

(D) Overtone=9

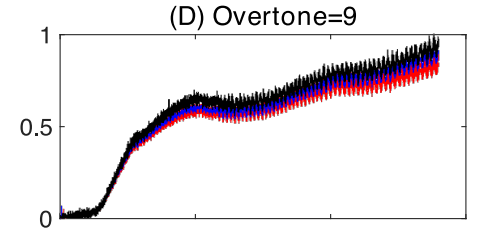

(F) Overtone $=13$

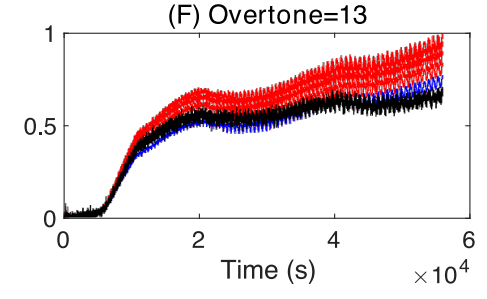

Figure 6.12: (A) Experimental half bandwidth shifts for all overtones. (B-F) Half bandwidth shifts per overtone from experiments (black), best model fit (blue) and 24 nearby combinations of the viscoelastic exponents (red).

\section{Fitted mass and compliances as a function of viscoelastic exponents}

To examine the uncertainties in the fit parameters (i.e. $m_{f}, J^{\prime}{ }_{r e f}$ and $J^{\prime \prime}{ }_{r e f}$, we calculate the Root Mean Squared Difference (RMSD):

$$
R M S D=\sqrt{\frac{1}{n} \sum_{n}\left(x_{\beta^{\prime}, \beta^{\prime \prime}}-x_{\text {best } \beta^{\prime}, \beta^{\prime \prime}}\right)^{2}}
$$

Where $x$ is the parameter for the choice of $\beta^{\prime}$ and $\beta^{\prime \prime}$ and the summation is over the whole experiment (as before). The RMSD shows us how far a predicted 
curve for a set of $\beta^{\prime}$ and $\beta^{\prime \prime}$ is from the curve predicted for the lowest RMSE. Figure 6.13 shows maps of the logarithm of the RMSD for $m_{f}, J^{\prime}$ ref and $J^{\prime \prime}$ ref . As expected from the form of Equation 6.6, the $m_{f}$ and $J^{\prime \prime}{ }_{r e f}$ RMSD depend mainly on $\beta^{\prime \prime}$ and the $J^{\prime}$ ref $\mathrm{RMSD}$ on $\beta^{\prime}$. The low values of the $m_{f}$ RMSD around the best fit compared to the mean $m_{f}$ throughout the experiment again confirm that $m_{f}$ is rather insensitive to the choice of the exponents.
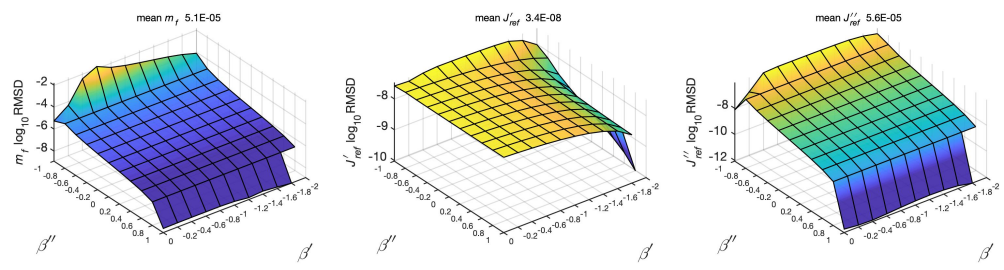

Figure 6.13: Maps of the logarithm of the Root Mean Squared Difference (RMSD) for Left: $m_{f}$, Center: $J^{\prime \prime}{ }_{\text {ref }}$ and Right: $J^{\prime}$ ref fitted over the whole experiment, as a function of the exponents $\beta^{\prime}$ and $\beta^{\prime \prime}$. Regions with a RMSD below $1 \%$ of the mean of the variable are in dark blue. For $J^{\prime}{ }_{\text {ref }}$ and $J^{\prime \prime}{ }_{\text {ref }}$ the first 1000 s are excluded from the RMSD calculation due to the small layer thickness and hence high uncertainty.

While the RMSD provides a good measure of how sensitively an extracted fit parameter depends on chosen constraints (i.e. the values taken for $\beta^{\prime}$ and $\beta^{\prime \prime}$ ), it does not show how this 'error' manifests in the time-dependent functions. In Fig. 6.14 we examine the outputs of $m_{f}, J^{\prime}$ ref and $J^{\prime \prime}{ }_{r e f}$ for different combinations of the exponents around the best choice. There is a negligible difference in the different $m_{f}$ curves, again indicating that the fitted value of $m_{f}$ should be accurate, given reasonable choices for the viscoelastic exponents. The $J^{\prime}{ }_{r e f}$ and $J^{\prime \prime}{ }_{r e f}$ curves on the other hand are visibly different. However, the different 
exponent choices just offset the curves. Thus, while their absolute values may be inaccurate, the trends in their values over the experiment should be reliable.
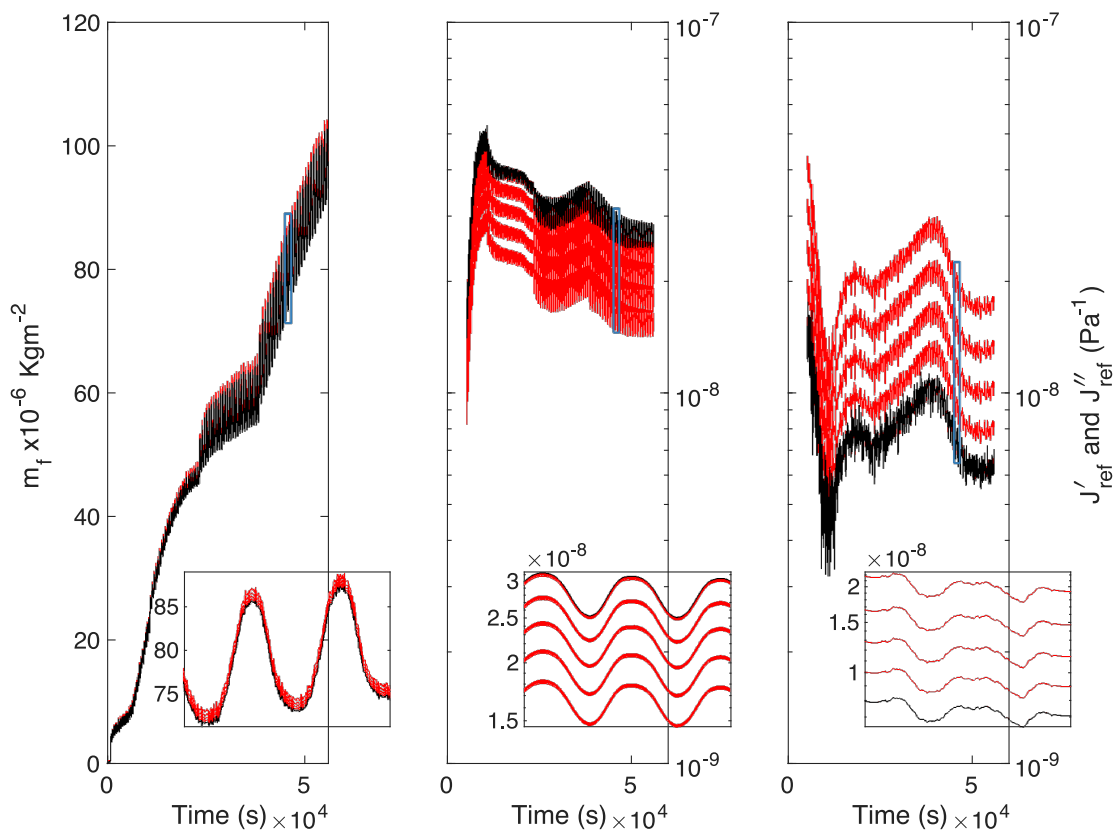

Figure 6.14: Extracted fit parameters (A) $m_{f},(B) J^{\prime \prime}{ }_{\text {ref }}$ and (A) $J^{\prime}{ }_{\text {ref }}$ for the best (black) and 24 next best (red) combinations of the viscoelastic exponents. Insets are a zoom of the boxed region. ${J^{\prime \prime}}^{\prime}{ }_{\text {ref }}$ and ${J^{\prime}}_{\text {ref }}$ curves have been SavitzGolay smoothed to aid visibility.

Fitted mass and compliances as a function of estimated layer density

We now consider the effect of the estimated layer density on the modelling results in Fig. 6.15. We performed the fitting for layer densities from 1000 to 2000 $\mathrm{Kgm}^{-3}$. Similar to the effect of the exponents, there is no effect of the layer 
density on $m_{f}$, while the $J^{\prime \prime}{ }_{\text {ref }}$ and $J^{\prime}{ }_{\text {ref }}$ curves are offset.
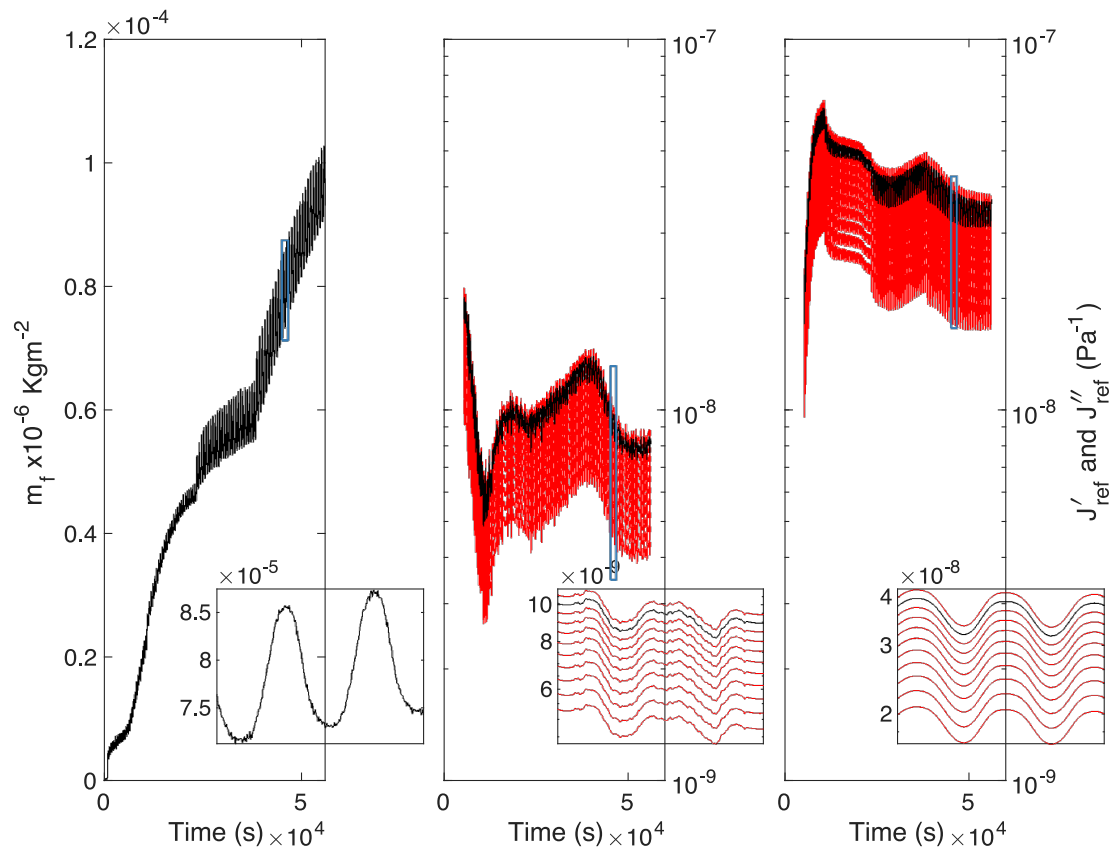

Figure 6.15: Model outputs $(A) m_{f},(B) J^{\prime \prime}{ }_{r e f}$ and $(C) J^{\prime}$ ref for eleven different layer densities (1000-2000 $\left.\mathrm{kgm}^{-3}\right)$. Insets are a zoom of the boxed region. $J^{\prime \prime}{ }_{\text {ref }}$ and $J^{\prime}{ }_{\text {ref }}$ curves have been Savitz-Golay smoothed to aid visibility.

\section{Effect of multilayers}

From the small load approximation, for a layer that has a compliance that varies with the thickness $z$, viz. a multilayer, if the densities are very close $\left(\rho_{f} \approx \rho_{l}\right)$ and the layer is thin ${ }^{14}$,

$$
\frac{\Delta \tilde{f}}{f_{0}} \approx \frac{-2 n \rho_{l} f_{0}}{Z_{q}} \int_{0}^{z_{t o t}}\left[1-i 2 \pi f_{0} \eta_{l} \tilde{J}_{f}(\omega, z)\right] d z
$$


From this equation, it is clear for a small viscous compliance $\left(\sim 1 \times 10^{-8} \mathrm{~Pa}^{-1}\right)$, the real part of the second term is small (order $10^{-4}$ ) and thus the effect of a multilayer structure on the predicted mass is very small. There will however be an effect on the predicted, now $z$-dependent, elastic compliance. Without prior knowledge on the actual multilayer structure, it is not possible to qualify this effect and the predicted compliance becomes an 'apparent compliance' for the multilayer as a whole.

\section{Effect of roughness}

The roughness found for typical SEI layers has a lateral scale $l_{r}$ much smaller than the shear wave penetration depth $\delta$. For a roughness of height $h_{r}$, assuming a rigid layer ${ }^{14}$,

$$
\frac{\Delta \tilde{f}}{f_{0}} \approx \frac{-1}{\pi Z_{q}} \sqrt{\pi f_{0} \rho_{l} \eta_{l}}\left[(1+i)+3 \sqrt{\pi} \frac{h_{r}^{2}}{l_{r} \delta}+2(i-1) \frac{h_{r}^{2}}{\delta^{2}}\right]
$$

The case $h_{r}=0$ corresponds to the bare crystal in liquid. For SEI, $h_{r}$ is of the order of a few $\mathrm{nm}, \delta$ close to $1000 \mathrm{~nm}$ and $l_{r} \mathrm{c}$ a few tens of nanometers ${ }^{1,41}$. Thus, the second two terms in the bracket are quite small leading to frequency shifts of a few to a few tens of Hertz and a bandwidth shift below 1 Hertz. This is well below the fitting error of the experiment and thus not expected to play a role. 


\subsubsection{Other Graphs}
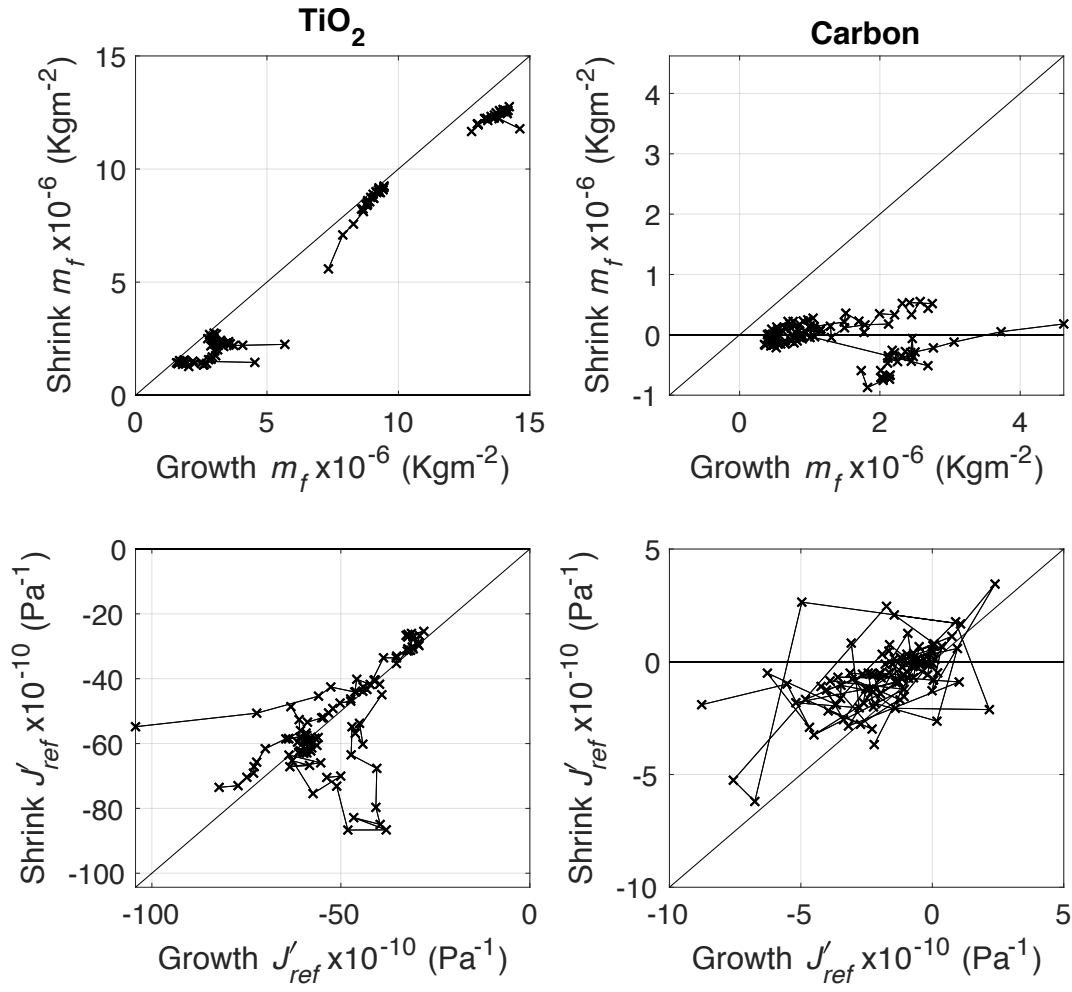

Figure 6.16: Correlation plots for the shrinkage and growth of $m_{f}$ and $J^{\prime}{ }_{r e f}$ for $\mathrm{TiO}_{2}$ and Carbon within the same charge-discharge cycle, based on Fig. 6.\%. The black line represents a 1:1 correlation. For $J^{\prime}{ }_{r e f}$ the first 4 cycles have been omitted. 

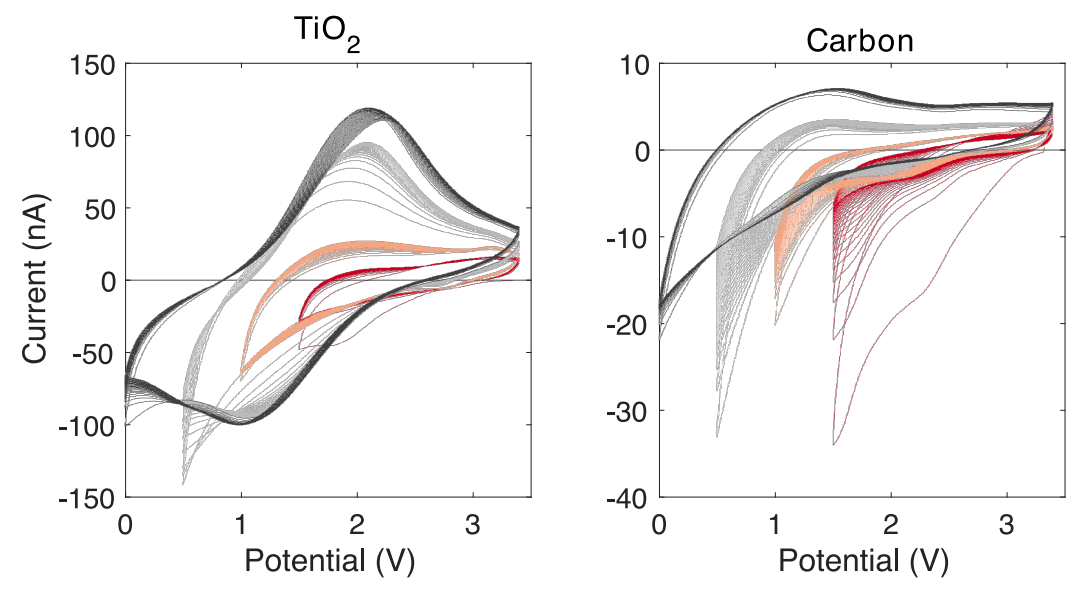

Figure 6.17: Cyclic Voltammograms for Left: $\mathrm{TiO}_{2}$ and Right: Carbon for the experiment in Figure 6.2 (main text). The voltage windows are $3.4 \mathrm{~V}$ to Red: 1.5 $V$ Teal: $1 \mathrm{~V}$ Gray: $0.5 \mathrm{~V}$ and Black: $0 \mathrm{~V}$. 


\section{Bibliography}

[1] D. Alliata, R. Kötz, P. Novák, and H. e. Electrochemical spm investigation of the solid electrolyte interphase film formed on hopg electrodes. Electrochemistry communications, 2(6):436-440, 2000.

[2] S. J. An, J. L. Li, C. Daniel, D. Mohanty, S. Nagpure, and D. L. Wood. The state of understanding of the lithium-ion-battery graphite solid electrolyte interphase (sei) and its relationship to formation cycling. Carbon, 105:52-76, 2016.

[3] D. Aurbach and A. Zaban. The application of eqcm to the study of the electrochemical-behavior of propylene carbonate solutions. Journal of Electroanalytical Chemistry, 393(1-2):43-53, 1995.

[4] S. Bhattacharya and A. T. Alpas. Micromechanisms of solid electrolyte interphase formation on electrochemically cycled graphite electrodes in lithiumion cells. Carbon, 50(15):5359-5371, 2012.

[5] D. Bresser, E. Paillard, E. Binetti, S. Krueger, M. Striccoli, M. Winter, and S. Passerini. Percolating networks of tio2 nanorods and carbon for high power lithium insertion electrodes. Journal of Power Sources, 206:301-309, 2012.

[6] H. Bryngelsson, M. Stjerndahl, T. Gustafsson, and K. Edström. How dynamic is the sei? Journal of Power Sources, 174(2):970-975, 2007.

[7] H. Buqa, R. I. R. Blyth, P. Golob, B. Evers, I. Schneider, M. V. S. Alvarez, F. Hofer, F. P. Netzer, M. G. Ramsey, M. Winter, and J. O. Besenhard. Negative electrodes in rechargeable lithium ion batteries - influence of graphite surface modification on the formation of the solid electrolyte interphase. Ionics, 6(3-4):172-179, 2000.

[8] D. A. Buttry and M. D. Ward. Measurement of interfacial processes at electrode surfaces with the electrochemical quartz crystal microbalance. Chemical Reviews, 92(6):1355-1379, 1992.

[9] A. C. Chu, J. Y. Josefowicz, and G. C. Farrington. Electrochemistry of highly ordered pyrolytic graphite surface film formation observed by atomic force microscopy. Journal of The Electrochemical Society, 144(12):41614169, 1997.

[10] A. v. Cresce, S. M. Russell, D. R. Baker, K. J. Gaskell, and K. Xu. In situ and quantitative characterization of solid electrolyte interphases. Nano letters, 14(3):1405-1412, 2014.

[11] V. Dargel, N. Shpigel, S. Sigalov, P. Nayak, M. D. Levis, L. Daikhin, and D. Aurbach. In situ real-time gravimetric and viscoelastic probing of surface films formation on lithium batteries electrodes. Nature Communications, 8 , 2017.

[12] M. Duduta, B. Ho, V. C. Wood, P. Limthongkul, V. E. Brunini, W. C. 
Carter, and Y. M. Chiang. Semi-solid lithium rechargeable flow battery. Advanced Energy Materials, 1(4):511-516, 2011.

[13] K. B. Hatzell, M. Boota, and Y. Gogotsi. Materials for suspension (semisolid) electrodes for energy and water technologies. Chemical Society Reviews, 44(23):8664-8687, 2015.

[14] D. Johannsmann. The quartz crystal microbalance in soft matter research. Fundamentals and modeling. Switzerland: Springer International Publishing, 2015.

[15] S.-P. Kim, A. C. Van Duin, and V. B. Shenoy. Effect of electrolytes on the structure and evolution of the solid electrolyte interphase (sei) in liion batteries: A molecular dynamics study. Journal of Power Sources, 196(20):8590-8597, 2011. (Chapter 2 of this thesis).

[16] P. G. Kitz, M. J. Lacey, P. Novak, and E. J. Berg. Operando eqcm-d with simultaneous in situ eis: New insights into interphase formation in li ion batteries. Analytical Chemistry, 91(3):2296-2303, 2019.

[17] H. Kumar, E. Detsi, D. P. Abraham, and V. B. Shenoy. Fundamental mechanisms of solvent decomposition involved in solid-electrolyte interphase formation in sodium ion batteries. Chemistry of Materials, 28(24):8930-8941, 2016.

[18] R. Lacroix, J. J. Biendicho, G. Mulder, L. Sanz, C. Flox, J. R. Morante, and S. Da Silva. Modelling the rheology and electrochemical performance of li4ti5o12 and lini1/3co1/3mn1/3o2 based suspensions for semi-solid flow batteries. Electrochimica Acta, 304:146-157, 2019.

[19] T. Liu, L. Lin, X. Bi, L. Tian, K. Yang, J. Liu, M. Li, Z. Chen, J. Lu, K. Amine, K. Xu, and F. Pan. In situ quantification of interphasial chemistry in li-ion battery. Nature Nanotechnology, 14(1):50-+, 2019.

[20] P. Lu, C. Li, E. W. Schneider, and S. J. Harris. Chemistry, impedance, and morphology evolution in solid electrolyte interphase films during formation in lithium ion batteries. Journal of Physical Chemistry C, 118(2):896-903, 2014.

[21] A. Narayanan, F. Mugele, and M. H. G. Duits. Mechanical history dependence in carbon black suspensions for flow batteries: A rheo-lmpedance study. Langmuir, 33(7):1629-1638, 2017. (Chapter 3 of this thesis).

[22] A. Narayanan, F. Mugele, and M. H. G. Duits. Device for rheometry, impedance spectroscopy, and electrochemistry on fluid electrodes. Review of Scientific Instruments, 90(2), 2019. (Chapter 4 of this thesis).

[23] A. Narayanan, D. Wijnperle, F. Mugele, D. Buchholz, C. Vaalma, X. Dou, S. Passerini, and M. H. G. Duits. Influence of electrochemical cycling on the rheo-impedance of anolytes for li-based semi solid flow batteries. Electrochimica Acta, 251:388-395, 2017. (Chapter 5 of this thesis).

[24] M. Y. Nie, D. P. Abraham, Y. J. Chen, A. Bose, and B. L. Lucht. Silicon 
solid electrolyte interphase (sei) of lithium ion battery characterized by microscopy and spectroscopy. Journal of Physical Chemistry C, 117(26):1340313412, 2013.

[25] M. Y. Nie, D. Chalasani, D. P. Abraham, Y. J. Chen, A. Bose, and B. L. Lucht. Lithium ion battery graphite solid electrolyte interphase revealed by microscopy and spectroscopy. Journal of Physical Chemistry C, 117(3):12571267, 2013.

[26] E. Peled and S. Menkin. Sei: past, present and future. Journal of The Electrochemical Society, 164(7):A1703-A1719, 2017.

[27] M. Pfanzelt, P. Kubiak, S. Jacke, L. Dimesso, W. Jaegermann, and M. Wohlfahrt-Mehrens. Sei formation on tio2 rutile. Journal of the Electrochemical Society, 159(6):A809-A814, 2012.

[28] S. F. Poli F, De Giorgio F. Flowable semi-solid electrodes with superconcentrated electrolytes: An electrochemical impedance study. Meeting Abstracts. The Electrochemical Society., 3:173-173, 2019.

[29] Y. Qi, L. G. Hector, C. James, and K. J. Kim. Lithium concentration dependent elastic properties of battery electrode materials from first principles calculations. Journal of the Electrochemical Society, 161(11):F3010-F3018, 2014.

[30] D. Rafieian, W. Ogieglo, T. Savenije, and R. G. H. Lammertink. Controlled formation of anatase and rutile tio2 thin films by reactive magnetron sputtering. Aip Advances, 5(9):7, 2015.

[31] J. J. Richards, A. D. Scherbarth, N. J. Wagner, and P. D. Butler. Mixed ionic/electronic conducting surface layers adsorbed on colloidal silica for flow battery applications. Acs Applied Materials \& Interfaces, 8(36):2408924096, 2016.

[32] M. Rodahl, F. Hook, A. Krozer, P. Brzezinski, and B. Kasemo. Quartzcrystal microbalance setup for frequency and q-factor measurements in gaseous and liquid environments. Review of Scientific Instruments, 66(7), 1995.

[33] M. Rodahl and B. Kasemo. A simple setup to simultaneously measure the resonant frequency and the absolute dissipation factor of a quartz crystal microbalance. Review of Scientific Instruments, 67(9):3238-3241, 1996.

[34] P. Sheng, E. K. Sichel, and J. I. Gittleman. Fluctuation-induced tunneling conduction in carbon-polyvinylchloride composites. Physical Review Letters, 40(18):1197-1200, 1978.

[35] R. D. Sherman, L. M. Middleman, and S. M. Jacobs. Electron-transport processes in conductor-filled polymers. Polymer Engineering and Science, 23(1):36-46, 1983.

[36] N. Shpigel, M. D. Levi, S. Sigalov, L. Daikhin, and D. Aurbach. In situ realtime mechanical and morphological characterization of electrodes for elec- 
trochemical energy storage and conversion by electrochemical quartz crystal microbalance with dissipation monitoring. Accounts of Chemical Research, 51(1):69-79, 2018.

[37] G. Shukla and A. A. Franco. Interphases in electroactive suspension systems: Where chemistry meets mesoscale physics. Batteries 65 Supercaps, 2(7):579590, 2019.

[38] E. K. Sichel, J. I. Gittleman, and P. Sheng. Transport properties of the composite-material carbon-poly(vinyl chloride). Physical Review B, 18(10):5712-5716, 1978.

[39] S. Sigalov, N. Shpigel, M. D. Levi, M. Feldberg, L. Daikhin, and D. Aurbach. Electrochemical quartz crystal microbalance with dissipation real-time hydrodynamic spectroscopy of porous solids in contact with liquids. Analytical Chemistry, 88(20):10151-10157, 2016.

[40] K. Singh and C. F. Blanford. Electrochemical quartz crystal microbalance with dissipation monitoring: A technique to optimize enzyme use in bioelectrocatalysis. Chemcatchem, 6(4):921-929, 2014.

[41] M. Steinhauer, M. Stich, M. Kurniawan, B.-K. Seidlhofer, M. Trapp, A. Bund, N. Wagner, and K. A. Friedrich. In situ studies of solid electrolyte interphase (sei) formation on crystalline carbon surfaces by neutron reflectometry and atomic force microscopy. ACS applied materials $\&$ interfaces, 9(41):35794-35801, 2017.

[42] N. W. Tschoegl. The phenomenological theory of linear viscoelastic behavior: an introduction. Springer Science \& Business Media, 2012.

[43] E. Ventosa, E. Madej, G. Zampardi, B. Mei, P. Weide, H. Antoni, F. La Mantia, M. Muhler, and W. Schuhmann. Solid electrolyte interphase (sei) at tio2 electrodes in li-ion batteries: defining apparent and effective sei based on evidence from x-ray photoemission spectroscopy and scanning electrochemical microscopy. ACS applied materials \& interfaces, 9(3):3123-3130, 2017.

[44] E. Ventosa, G. Zampardi, C. Flox, F. La Mantia, W. Schuhmann, and J. R. Morante. Solid electrolyte interphase in semi-solid flow batteries: a wolf in sheep's clothing. Chemical Communications, 51(81):14973-14976, 2015.

[45] P. Verma, P. Maire, and P. Novak. A review of the features and analyses of the solid electrolyte interphase in li-ion batteries. Electrochimica Acta, 55(22):6332-6341, 2010.

[46] S. W. Wang, K. Yang, F. Gao, D. Y. Wang, and C. Shen. Direct visualization of solid electrolyte interphase on li4ti5o12 by in situ afm. Rsc Advances, 6(81):77105-77110, 2016.

[47] Z. Yang, B. J. Ingram, and L. Trahey. Interfacial studies of li-ion battery cathodes using in situ electrochemical quartz microbalance with dissipation. Journal of the Electrochemical Society, 161(6):A1127-A1131, 2014.

[48] G. Zampardi, E. Ventosa, F. La Mantia, and W. Schuhmann. In situ visual- 
ization of li-ion intercalation and formation of the solid electrolyte interphase on tio2 based paste electrodes using scanning electrochemical microscopy. Chemical Communications, 49(81):9347-9349, 2013.

[49] S. S. Zhang, M. S. Ding, K. Xu, J. Allen, and T. R. Jow. Understanding solid electrolyte interface film formation on graphite electrodes. Electrochemical and Solid State Letters, 4(12):A206-A208, 2001.

[50] Y. Zhang, A. Narayanan, F. Mugele, M. A. C. Stuart, and M. H. Duits. Charge inversion and colloidal stability of carbon black in battery electrolyte solutions. Colloids and surfaces A: Physicochemical and engineering aspects, 489:461-468, 2016.

[51] J. Zheng, H. Zheng, R. Wang, L. Ben, W. Lu, L. Chen, L. Chen, and H. Li. 3d visualization of inhomogeneous multi-layered structure and young's modulus of the solid electrolyte interphase (sei) on silicon anodes for lithium ion batteries. Physical Chemistry Chemical Physics, 16(26):13229-13238, 2014. 
Chapter 7

\title{
Conclusions and Outlook
}

\author{
ADITyA NARAYANAN
}




\subsection{Conclusions}

In this thesis, we investigated many of the aspects of fluid electrodes brought on by moving to the colloidal domain using a variety of macroscopic and microscopic techniques. In this section we present the highlights of our findings.

First we studied the colloidal interactions of Ketjen Black particles in dilute electrolyte solutions using dynamic light scattering. The particles were found to be negatively charged and stable (against agglomeration) in the absence of salt. On addition of salt the particles agglomerated. For $\mathrm{LiPF}_{6}$ and LiTFSI, on further addition of salt, a second stability regime was found. This re-entrant stability was not found for $\mathrm{NaPF}_{6}$. We attribute this behavior to selective adsorption of lithium cations which reverse the particle zeta potential and increase its magnitude. As salt concentration increased further, the particles again agglomerate, tending towards diffusion limited agglomeration as the salt concentration reached those used in typical battery electrolyte solutions.

Subsequently we shifted our attention to the higher, practically relevant, particle fraction regime. We studied the rheology and electronic conductivity of relatively concentrated ( $\sim 1 \%$ wt.) Ketjen Black suspensions (dispersed in electrolyte) in a home-built 'Rheoimpedance Setup'. We found a two timescale agglomeration process that determined the microstructure, and consequently the rheology and electronic conductivity of the suspensions. At equilibrium, higher shear rates led to smaller agglomerates and even primary indivisible aggregates at high enough shear rates. On stepping down the shear rate, an immediate open agglomeration was observed with an increase in viscosity. As shearing was continued, the viscosity and electronic conductivity decreased, indicating a densification of the agglomerates, presumably due to interpenetration. Steps in the 


\subsection{CONCLUSIONS}

reverse direction were accompanied with the reversed the effect. On cessation of shear, there was an immediate formation of a space spanning percolated network. This network was interpreted to be built of the 'frozen-in' agglomerates just before cessation. High pre-shears resulted in greater electronic conductivity and higher mechanical strength (yield stress). Comparisons with suspensions of spherical carbon black particles showed that the fractal particle morphology was the source of this behavior.

The strong mechanical history dependance of the suspensions meant that studying their electrochemistry requires precise mechanical control. To achieve this, we developed a rheometer modification that allows simultaneous rheometry, impedance spectroscopy and electrochemical control. The device consists of a plate plate shearing geometry for the fluid electrode under study, with a counter electrode below the bottom shearing plate. The counter electrode can either be a 'reference' lithium electrode or a second fluid electrode. We demonstrated the device on multiple fluid electrode systems, all of which displayed strong mechanical and electrochemical history dependent effects.

Armed with this custom setup, we isolated the influence of electrochemical state and history by establishing a consistent mechanical state. We found that SSFB anolytes display a state-of-charge dependent rheology and electronic conductivity. Charging the anolytes was found to decrease both the mechanical strength and conductivity. Discharging was found to (partially) restore both. Charging to more aggressive (reducing) voltages resulted in a growing irreversible loss in strength and conductivity. Experiments with suspensions of only Ketjen Black showed similar results. We inferred these trends to be due to the formation of a partially reversible insulating SEI layer on the particle surfaces. SEI 
was found to form even at conservative operating voltages, which are generally thought to be free of it. The SEI's impact on the fluid electrodes was drastic, with the extracted electronic conductivity worsening by multiple orders of magnitude in many cases. Such layer formation in conservative operating windows, while inconsequential to traditional batteries, was identified to be potentially catastrophic to flow systems

Finally, we used the Quartz Crystal Microbalance with Dissipation to directly monitor the formation of these surface layers. We used thin films of $\mathrm{TiO}_{2}$ and carbon as analogues of active and conductive nanoparticle surfaces. During repeated electrochemical cycling, both layers showed mass growth while the titania showed mass oscillations in addition. Comparisons with the injected charge and extracted viscoelasticity of the layers showed that titania showed both reversible lithium intercalation and irreversible SEI formation while carbon only showed SEI formation. Again SEI was (directly) observed at potentials generally considered free from it. Experiments using a second electrolyte and carbon showed similar SEI formation.

\subsection{Outlook}

We see our findings having implications for both traditional and fluid electrode systems. Our observations on the colloidal interactions and mechanical history dependance of carbon black suspensions are useful for optimizing operation and pumping strategies of fluid electrode systems. They can also be used to optimize electrode manufacture for traditional batteries where similar suspensions are sheared during coating, calendering, etc. In real systems however, there is 


\subsection{OUTLOOK}

additional complexity due to the active particles. the additional self and cross interactions can have significant effects on the microstructure and behavior. Understanding these interactions is therefore important to optimize the mechanical handling of fluid electrodes.

We believe that our 'Cycling Rheoimpedance' setup, while specialized, is an essential tool to take into account (in a reproducible manner) the effects of mechanical history on studies of fluid electrodes. Most studies of fluid electrodes currently use either a (slurry filled) static cell or a flow channel based device, both of which do not accurately control the mechanical state and history. The transition to versions of our setup would greatly help reduce irreproducibility in experimental studies.

Our observations of SEI formation in regimes where unexpected, highlight the need for careful chemistry choices for fluid electrodes. We demonstrate the Quartz Crystal Microbalance with Dissipation as a sensitive and powerful method for quickly screening different systems.

While SSFBs show immense potential, the chemistry of typical materials used brings about a catch-22. For high energy densities, high operation voltages necessitate SEI formation. Even minor SEI formation, due to the dynamic microstructure, kills fluid electrode performance. Two possible solutions to avoid SEI are to operate at lower voltages or to use materials that do not form or need SEI. The first is not an ideal solution as it diminishes the main advantage of SSFBs over redox flow batteries; energy density. Additionally, as we have shown, many materials form thin SEI even at relatively low operating voltages. The second requires the exploration of novel chemistries and material combinations, especially of the electrolyte solution. This approach would have the highest po- 
tential as most currently proposed SSFB materials are the same as those used in traditional solid batteries. LIB material choices are driven by a compromise to many requirements, some of which are very different in SSFBs. 


\section{Summary}

Semi Solid Flow Batteries (SSFBs) are a promising architecture of lithium ion batteries where slurries of nanoparticles are used in place of solid anodes and cathodes. The move to fluidic electrodes brings about many challenges such as the effect of shear, gravity, etc. In this thesis, we investigate some of the challenges brought on by the now colloidal particles and their interactions and assembly.

In Chapter 2 we investigate the colloidal interactions of the dilute conductive particles (Ketjen Black) in electrolyte. Using dynamic light scattering and electrophoretic mobility measurements, we find a salt and salt concentration dependent stability (against flocculation) and zeta potential. We find that the particle zeta potential goes from negative to positive and then down to zero as the salt concentration is increased. At the very high salt concentrations found in batteries, the particles are completely electrostatically screened by the salt and agglomeration is diffusion limited.

In Chapter 3 we simultaneously study the rheology and electronic conductivity of concentrated, percolated ketjen black suspensions. Starting with broken up agglomerate particles during shear, we find that a change in shear rate leads to a two timescale response related to the branched morphology of the unit particles 
and their agglomeration behavior. For steps down in the rate open agglomerates immediately form with an increase in viscosity. With time the agglomerates densify leading to a reduction in the viscosity and conductivity. Additionally the mechanical strength and conductivity on cessation of shear depend on the shearing rate just before, with higher rates leading to higher values of both. This tune-ability of important properties has strong implications for pumping strategies of fluid electrodes.

In Chapter 4 we describe the design of an instrument that allows us to control (and measure) both the mechanical and electrochemical state of fluid electrodes simultaneously. We demonstrate the instrument on multiple types of fluid electrodes almost all of which show strong mechanical and electrochemical history dependance.

In Chapter 5 we use the device to study the effect of electrochemical history on SSF anodes. On both typical and conductive particle only fluid electrodes, we find SEI layer formation, where none is expected. The SEI negatively affects both the rheology and electronic conductivity. This layer was dynamic; it was formed on charge and partially reversed on discharge. More aggressive voltages led to more insulating and more permanent layers, with catastrophic effects on the fluid electrodes. Fitted electronic resistances increased by multiple orders of magnitude even at voltages less reducing than those used in conventional solid systems.

In Chapter 6 we directly observed this layer formation using the Quartz Crystal Microbalance. We used $\mathrm{TiO}_{2}$ and Carbon thin films as analogues for active and conductive particle surfaces. Using a viscoelastic model and comparing current flows, we differentiated between lithium intercalation and SEI formation. 
Carbon shows only SEI formation, while $\mathrm{TiO}_{2}$ shows both reversible intercalation and SEI. Again, SEI is observed on both materials in 'mild' voltages where none is expected. 


\section{Samenvatting}

Semi Solid Flow Batterijen (SSFB's) zijn een veelbelovende configuratie van lithium-ionbatterijen, waarbij een brij van nanodeeltjes wordt gebruikt ter vervanging van vaste anoden en kathoden. De overstap naar vloeibare elektroden brengt veel uitdagingen met zich mee, zoals het effect van afschuifkrachten, zwaartekracht, enz. In dit proefschrift onderzoeken we enkele van de uitdagingen, die de nu collö̈dale deeltjes - en hun interacties en aggregatie - met zich meebrengen.

In Chapter 2 onderzoeken we de colloïdale interacties van verdunde, geleidende deeltjes (Ketjen Black) in elektrolyt. Met behulp van dynamische lichtverstrooiing en elektroforetische mobiliteitsmetingen vinden we een zout- en zoutconcentratieafhankelijke stabiliteit (tegen vlokvorming) en zeta-potentiaal. We ontdekken dat de zeta-potentiaal van de deeltjes van negatief naar positief gaat en vervolgens naar nul daalt naarmate de zoutconcentratie wordt verhoogd. Bij de zeer hoge zoutconcentraties, die in batterijen worden gebruikt, worden de deeltjes volledig elektrostatisch afgeschermd door het zout en wordt agglomeratie beperkt door diffusie.

In Chapter 3 bestuderen we tegelijkertijd de reologie en elektrische geleidbaarheid van geconcentreerde, gepercoleerde Ketjen Black suspensies. Begin- 
nend met door afschuiving opgebroken agglomeraatdeeltjes zien we dat een verandering in afschuifsnelheid leidt tot een respons op twee tijdschalen, die verband houden met de vertakte morfologie van de eenheidsdeeltjes en hun agglomeratiegedrag. Bij stapsgewijze afname in snelheid worden onmiddellijk open agglomeraten gevormd met een toename in viscositeit tot gevolg. Na verloop van tijd verdichten de agglomeraten, hetgeen leidt tot een vermindering van de viscositeit en geleidbaarheid. Bovendien hangen de mechanische sterkte en geleidbaarheid bij het stoppen van afschuiving af van de afschuifsnelheid net daarvoor, waarbij hogere snelheden tot hogere waarden van beide leiden. Deze controleerbare verandering van fysische eigenschappen heeft belangrijke gevolgen voor pompstrategieën voor vloeibare elektrodes.

In Chapter 4 beschrijven we het ontwerp van een instrument, waarmee we zowel de mechanische als de elektrochemische toestand van vloeibare elektrodes tegelijkertijd kunnen regelen (en meten). We demonstreren het instrument op meerdere types vloeibare elektrodes, welke bijna allemaal een sterke mechanische en elektrochemische geschiedenisafhankelijkheid vertonen.

In Chapter 5 gebruiken we hetzelfde instrument om het effect van de elektrochemische geschiedenis te bestuderen. Op zowel standaard als alleen-geleidendedeeltjes vloeistofelektroden vinden we SEI-laagvorming, waar geen laagvorming wordt verwacht. De SEI heeft een negatieve invloed op zowel de reologie als de elektronische geleidbaarheid. Deze laag is dynamisch, wordt gevormd bij oplading en wordt gedeeltelijk weer afgebroken bij ontlading. Meer agressieve spanningen leidden tot meer, en meer permanente lagen - met catastrofale gevolgen voor de vloeibare elektroden. Gemeten elektronische weerstanden namen met meerdere ordes van grootte toe, zelfs bij spanningen die minder reduceren dan die gebruikt 
in conventionele vaste systemen.

In Chapter 6 hebben we deze laagvorming direct waargenomen met behulp van de Quartz Crystal Microbalance (QCM). We gebruikten dunne lagen $\mathrm{TiO}_{2}$ en koolstof als analogen voor actieve en geleidende deeltjesoppervlakken. Met behulp van een visco-elastisch model en door het vergelijken van stroomtoevoer, maakten we onderscheid tussen intercalatie en SEI-vorming. Koolstof vertoont alleen SEI-vorming, terwijl $\mathrm{TiO}_{2}$ zowel omkeerbare intercalatie als SEI vertoont. Opnieuw wordt SEI op beide materialen waargenomen bij 'milde' spanningen waar geen laagvorming wordt verwacht. 


\section{Acknowledgements}

During a large majority of the last few years, I had fantasized about being at the stage of writing this section. I had dreamt of this moment every time I read the acknowledgements of my peers before me. Writing my acknowledgements meant that I was about to cross the finish line. Now that I am finally here, I'm filled with mixed feelings which a few years ago I would not have expected. As I reflect, my takeaway is that despite the many negatives I did learn a lot; and that alone makes this experience a net positive.

I would like to start by acknowledging the contribution of my daily supervisor, Michel. You helped me get out of many ruts, by providing a second set of eyes. I am extremely grateful for your ability to put things in context and look at the 'bigger picture'. I also appreciate the way you helped structure my research into appealing 'stories' for publication. I had a lot of fun travelling with you to various conferences and meetings during the course of my project.

I would like to thank Frieder for the opportunity to work at PCF, with its excellent facilities. I also thank you for including me in various projects outside my thesis, and thus exposing me to a much broader variety of research areas.

Dirk, though I never directly worked with you, I was always enamoured by your approach to science. Your ability to break things into the right 'simpler 
problems' is something I have great respect for.

Martien (Stuart), a big thank you for the many discussions on the colloidal side of my project.

I also thank my fellow 'Influencers', Yan, Duc, and the various collaborators outside UTwente for their contributions to the chapters.

While doing a PhD, a large majority of days (and nights) are spent in the lab, so a large thanks to my colleagues who were also my friends during the last few years. I am sure I will miss mentioning many, but be assured I enjoyed the pointless arguments and discussions with all of you. A special thanks to my favourite colleague Arjen whose colourful jokes and opinions livened up the group. Aram, Martin, Sachin and Simone, the Happy Italy and drinking evenings were always enjoyable and a good way to vent frustrations. Some of those conversations did funnily have a profound effect on my life. Daniel, thanks for the always prompt help with my samples and the many gym and badminton sessions. Our 'discussions' brightened countless days. Nicolao, our 'just one beer' all nighters... well, I don't remember much more to say here. And my many other lab-mates; Igor, Beybin, Natalie, Carla, Stelian, Jun, Ranabir, Somnath, Omkar, Isabel, Olena, Querie, Daniel H, Davood, Rudy, Zhantao and the others, whom I've forgotten. Of course a special mention to my 'Indian' group; Naveen, Mitava, Karthikeya, Harshita, Manas, Shantanu, Poorvi.... Our many many dinners, nights losing money in the casino, mafia, etc. were and still remain highlights of my (read only) social life.

My final acknowledgements go to my family. My parents for instilling my spirit of endless curiosity. My mother for her unconditional support. The rest also for their support (and nagging). And finally my wife Karthika; thank you 
for dealing with my past baggage i.e. my unfinished thesis. I probably would have quit writing if not for your constant pushing.

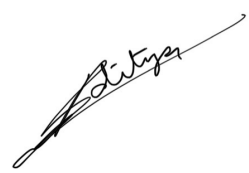




\section{TL;DR}

Aditya NarayAnAN

- Batteries are tough. Flowing ones even more so.

- Everything Agglomerates.

- Conductive Carbons are weird and stick to each other in weird ways thanks to their shape.

- Shear like hell; you get stronger and better conducting networks.

- Fortunately for lithium batteries, insulating ion permeable layers form on everything.

- Unfortunately for semi solid flow batteries, insulating ion permeable layers form on everything. 


\section{Publications}

Mishra, K., Narayanan, A., and Mugele, F. (2019). Design and wavefront characterization of an electrically tunable aspherical optofluidic lens. Optics Express, 27(13):17601-17609.

Narayanan, A., Mugele, F., and Duits, M. H. (2017a). Mechanical history dependence in carbon black suspensions for flow batteries: A rheo-impedance study. Langmuir, 33(7):1629-1638.

Narayanan, A., Mugele, F., and Duits, M. H. (2019). Device for rheometry, impedance spectroscopy, and electrochemistry on fluid electrodes. Review of scientific instruments, 90(2):025112.

Narayanan, A., Mugele, F., and Duits, M. H. (2020). Electrochemically induced changes in tio2 and carbon films, studied with qcm-d. Advanced Energy Materials. Accepted.

Narayanan, A., Wijnperle, D., Mugele, F., Buchholz, D., Vaalma, C., Dou, X., Passerini, S., and Duits, M. H. (2017b). Influence of electrochemical cycling on the rheo-impedance of anolytes for li-based semi solid flow batteries. Electrochimica acta, 251:388-395.

Zhang, Y., Narayanan, A., Mugele, F., Stuart, M. C., and Duits, M. H. (2016). Charge inversion and colloidal stability of carbon black in battery electrolyte solutions. Colloids and surfaces A: Physicochemical and engineering aspects, 489:461-468. 


\section{About the Author}

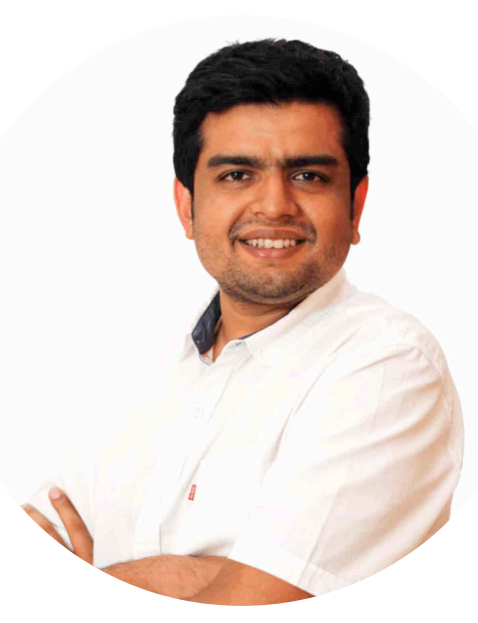

Aditya Narayanan was born in Bangalore, India on the $11^{\text {th }}$ of March 1990. After finishing his schooling, he went on to complete a 5 year combined bachelor and masters degree at IIT-Varanasi. Shortly after, he joined the Physics of Complex Fluids group at the University of Twente for his $\mathrm{PhD}$ research. 